\title{
UNBIASED FUNCTIONAL PROTEOMICS TO IDENTIFY NOVEL MEDIATORS OF TUMOR VESSEL DEVELOPMENT
}

\begin{tabular}{l} 
A dissertation submitted to the Faculty of \\
The School of Engineering and Applied Science \\
University of Virginia \\
\hline
\end{tabular}

In partial fulfillment of the requirements for the degree of

Doctor of Philosophy in Biomedical Engineering

By

Marc Edward Seaman

May 


\section{APPROVAL SHEET}

This dissertation is submitted in partial fulfillment of the requirements for the degree of Doctor of Philosophy in Biomedical Engineering

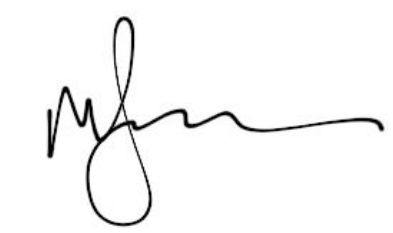

Marc E. Seaman, Author

This dissertation has been read and approved by the Examining Committee:

Shayn Peirce-Cottler, Ph.D., Chair of Committee

Kimberly Kelly, Ph.D., Thesis Advisor

Richard Price, Ph.D.

Brent French, Ph.D.

Todd Bauer, M.D.

Accepted for the School of Engineering and Applied Science:

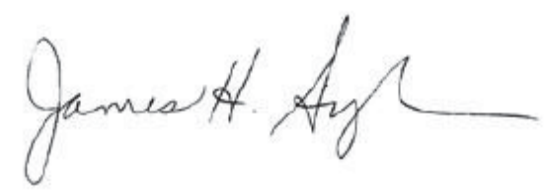

Dean, School of Engineering and Applied Science

May 


\section{ACKNOWLEDGEMENTS}

First and foremost, I would like to thank my wonderful advisor, Dr. Kimberly

Kelly, for her mentorship throughout my graduate career. She has been a constant supporter and our families have grown to become great friends, reinforcing the notion that there is more to life than just lab work. In fact, the phrase "work hard, play hard" is the unofficial motto of the Kelly Lab. Speaking of, I would like to thank all of the members of the Kelly Lab for being so willing to listen and provide scientific or life advice. I never once dreaded going to work and the lab members have a lot to do with that. I'd also like to thank my classmates from the Fall 2008 entering class for the scientific discussions and most importantly, the great experiences we shared.

To my parents, Jeff and Linda - thanks for always being your son's biggest fans. Your constant love and support of my graduate pursuits has made it easy to accomplish the work presented in this dissertation. Without such a great upbringing, none of this would be possible. To my brother Scott and his wife Stephanie, your love and friendship has made living away from "home" much easier.

Finally, I want to whole-heartedly thank my wife Amanda for all of her love and support through the years. I can't imagine how difficult it must be to be the spouse of someone going through a loosely-defined progression of milestones on the path to obtaining a Ph.D. I wouldn't have rather had anyone else to share both the frustrations and elations that come with being a graduate student. I look forward to our next chapter. I love you. 


\section{TABLE OF CONTENTS}

ABSTRACT

LIST OF TABLES V vii

LIST OF FIGURES V vii

CHAPTER 1: INTRODUCTION 1

1.1 Introduction 2

1.1.1 Cancer: A Public Health Concern in Need of Improved Therapies 2

1.1.2 Cancer is Dependent on Neovascularization 2

1.1.3 Angiogenesis: Physiologic and Pathologic 3

1.1.3.1 Angiogenic Factors 4

1.1.3.1.1 Vascular Endothelial Growth Factor (VEGF) 5

1.1.3.1.2 Fibroblast Growth Factor (FGF) 6

$\begin{array}{ll}\text { 1.3.1.1.3 Other angiogenic factors } & 7\end{array}$

1.1.4 Anti-vascular therapy 8

1.1.4.1 Anti-VEGF therapies 9

1.1.4.1.1 Monoclonal antibodies against VEGF or VEGFR2 9

1.1.4.1.2 Small molecule inhibitors of VEGF or VEGFR $\quad 10$

1.1.4.2 Other angiogenic factor inhibitors $\quad 12$

1.1.5 Modest survival resulting from resistance to anti-vascular therapy 12

1.1.5.1 Innate Resistance 13

$\begin{array}{ll}\text { 1.1.5.2 Acquired resistance } & 15\end{array}$

1.1.6 Discovery of additional VEGF-independent angiogenic factors $\quad 16$

1.1.6.1 Approaches to identify VEGF-independent factors 16

1.1.6.1.1 Genomics methods 17

1.1.6.1.1.1 Comparative genome sequencing $\quad 17$

1.1.6.1.1.2 Differential gene expression analysis $\quad 18$

1.1.6.1.2 Proteomics methods 20

1.1.6.1.2.1 2D-PAGE or 2D-DIGE methods 20

1.1.6.1.2.2 Phage display $\quad 22$

1.1.6.1.2.3 Functional proteomics 23 
CHAPTER 2: HORNERIN IDENTIFIED AS A NON-VEGF MEDIATED FACTOR THROUGH PHAGE DISPLAY BASED FUNCTIONAL PROTEOMICS

2.1 Abstract

2.2 Introduction

2.3 Results and Discussion

2.3.1 Selected phage clones are specific for tumor vasculature in vivo

2.3.2 Selected phage clones are specific for TCM treated HUVECs

2.3.3 Hornerin identified as the binding partner of PTEM 9

2.3.4 Hornerin is upregulated in HUVECs in response to TCM but not VEGFCM

2.3.5 Hornerin is upregulated in HUVECs in response to interleukin-12 (IL12) and basic fibroblast growth factor (FGF2)

2.3.6 Hornerin is specifically expressed in human PDAC vessels

2.4 Materials and Methods

2.4.1 Statistics

2.4.2 Cell culture

2.4.3 Phage screen and sequencing

2.4.4 Phage-based ELISA

2.4.5 Two-way ANOVA analysis

2.4.6 HUVEC lysates and immunocytochemistry

2.4.7 Tumor conditioned media and implantation

2.4.8 FITC-labeling of phage clones

2.4.9 Western Blot and Angiogenesis Antibody Array

2.4.10 De-identified human PDAC sample collection, immunohistochemistry, and pathologic scoring

CHAPTER 3: HORNERIN EXPRESSION IS IMPORTANT IN TUMOR PROGRESSION AND VESSEL DEVELOPMENT 
3.2 Introduction 57

3.3 Results and Discussion $\quad 59$

3.3.1 Detecting angiogenic changes in vessel architecture using RAVE $\quad 59$

3.3.2 Knockdown of hornerin leads to decreased tumor burden and key vessel parameters

3.3.3 Functional imaging reveals reduced vascularity and perfusion in hornerin siRNA treated tumors.

3.3.4 VEGF inhibitor AV-951 combined with hornerin knockdown produced an additive reduction in tumor growth and vessel development. 69

$\begin{array}{ll}\text { 3.4 Materials and Methods } & 74\end{array}$

3.4.1 Tumor Implantation $\quad 74$

3.4.2 siRNA injections $\quad 74$

3.4.3 Tumor volume measurement, immunofluorescence and vessel parameter quantification. $\quad 75$

3.4.4 AV-951 administration $\quad 76$

3.4.5 Microscopy 76

3.4.6 DCE-MRI and Ktrans $\quad 77$

CHAPTER 4: HORNERIN EXPRESSION CONFERS RESISTANCE TO OXIDATIVE STRESS THROUGH ACTIVATION OF NF-kB 79

$\begin{array}{ll}4.1 \text { Abstract } & 80\end{array}$

$\begin{array}{ll}4.2 \text { Introduction } & 81\end{array}$

$\begin{array}{ll}4.3 \text { Results and Discussion } & 82\end{array}$

4.3.1 EGFP-hornerin fusion protein exhibits a similar expression pattern compared to TCM-treated HUVECs 82

4.3.2 Hornerin expression confers resistance to oxidative stress 83

4.3.3 Hornerin expression results in activation of NF-kB and subsequent BCL-2 localization to mitochondria $\quad 87$

4.3.4 Incubation with JSH-23, an inhibitor of NF-kB nuclear translocation reduces NF-kB translocation, BCL-2/mitochondria localization and protection from oxidative stress in hornerin expressing cells. 
4.4.1 Recombinant Hornerin Expression

4.4.2 Annotated sequence of engineered DNA construct supplied by Genscript

4.4.3 NF-kB, BCL-2 and BAX analysis

4.4.4 Hydrogen peroxide and JSH-23 treatment

4.4.5 TUNEL analysis 


\section{ABSTRACT}

Most current and past preclinical and clinically approved anti-tumor vessel therapies focus on the vascular endothelial growth factor (VEGF) pathway. Although targeting VEGF has proven effective in certain cancers, overall, the clinical efficacy has been modest at best. Where efficacious, tumors initially respond then recur becoming resistant to VEGF inhibitors in the process. Therapies against non-VEGF mediated proteins that might augment VEGF inhibition could hold the key to improving patient survival. Therefore, we identified VEGF-independent factors through a combination of in vivo phage display in mice with orthotopically implanted pancreatic ductal adenocarcinoma (PDAC) tumors and functional proteomics methods. Hornerin, an S100 family member, was identified and subsequently validated via immunofluorescence and immunoblotting as a protein important in tumor endothelial cells in vitro and in vivo. In human PDAC vessels, hornerin was highly expressed, indicating potential clinical relevance. Knockdown of tumor vessel hornerin by intratumoral injections of siRNA reduced tumor volume and important vessel parameters as measured by software we developed and published called Rapid Analysis of Vessel Elements (RAVE). Functionally, dynamic contrast-enhanced magnetic resonance imaging (DCE-MRI) revealed reduced vascularity and perfusion in hornerin knockdown tumors. Further, anti-hornerin therapy in combination with a VEGF receptor inhibitor AV-951 resulted in additive decreases in tumor volume, providing evidence that targeting independent pathways may result in enhanced therapeutic efficacy.

Mechanistically, we showed that hornerin expression contributes to protection 
from oxidative stress through NF- $\mathrm{KB}$ induced BCL2 localization to the mitochondria. This protective effect was eliminated after incubation with an NF- $\mathrm{kB}$ inhibitor. The identification of hornerin, a VEGF-independent protein important in tumor vessel development and endothelial survival, provides a potential therapeutic target to be used alone or in combination with existing VEGF inhibitor therapy. 


\section{LIST OF TABLES}

Table 2.1 Characteristics of PDAC patients used in this study.

\section{LIST OF FIGURES}

Figure 2.1 Selected phage clones are specific for tumor vasculature in vivo. 30

Figure 2.2 Two-way ANOVA analysis identifies TCM specifically upregulated clones.

Figure 2.3 Several PTEM clones specifically bind TCM, but not VEGFCM treated HUVECs.

Figure 2.4 Hornerin identified as the binding partner of PTEM 9.

Figure 2.5 Hornerin is present on the cell surface of TCM treated HUVECs. 37

Figure 2.6 Hornerin is upregulated in TCM treated HUVEC whole cell lysates.

Figure 2.7 Hornerin is upregulated in HUVECs in response to interleukin-12 (IL12) and basic fibroblast growth factor (FGF2).

Figure 2.8 Hornerin is expressed in resected human PDAC associated tumors and vessels.

Figure 2.9 Hornerin is expressed in a variety of solid tumors.

Figure 2.10 Quantification of PDAC and normal pancreas vessels.

$\begin{array}{lll}\text { Figure 3.1 } & \text { Overview of RAVE GUI. }\end{array}$

Figure 3.2 Hornerin knockdown results in decreased tumor volume. 61

Figure 3.3 Hornerin knockdown results in decreased VVF and fractal dimension, but no change in the number of vessels per field. $\quad 62$

Figure 3.4 Hornerin knockdown results in a shift to smaller vessel radii. $\quad 64$

Figure 3.5 Hornerin knockdown increases ACTA2-positive pericyte coverage. 65 
Figure 3.6 Functional studies by dynamic contrast-enhanced magnetic resonance imaging (DCE-MRI) reveal decreased vascularity in $H r n r$ siRNA treated tumors.

Figure 3.7 Gadolinium-DTPA (Gd-DTPA) uptake and volume transfer coefficient $\mathrm{K}_{\text {trans }}$ are reduced in $\mathrm{Hrnr}$ siRNA treated tumors.

Figure 3.8 Hornerin and VEGF combination treatment results in increased tumor volume and tumor vessel abatement.

Figure 3.9 AV-951 and hornerin combination therapy produces vessels with varying morphology.

Figure 3.10 AV-951, in combination with hornerin siRNA therapy enhances anti-vessel effects.

Figure 4.1 Engineered EGFP-HRNR expression matches TCM-induced HUVEC expression.

Figure 4.2 EGFP-HRNR cells protected from oxidative stress.

Figure 4.3 Hornerin expression induces NF- $\mathrm{kB}$ activation and nuclear translocation.

Figure 4.4 Hornerin expression induces BCL-2/mitochondrial localization.

Figure 4.5 NF- $\kappa B$ inhibitor of nuclear translocation prevents NF- $\kappa B$ translocation and disrupts BCL-2/mitochondrial localization.

Figure 4.6 Treatment with JSH-23 eliminates hornerin mediated protection from oxidative stress.

Figure 5.1 Hornerin is characterized by the EF-hand and tandem repeat domains and shares homology with other S100 family members. 103

Figure 5.2 Hornerin could potentially act at several points in the NF- $\mathrm{KB}$ activation cascade.

Figure 5.3 Hornerin knockdown reduces L3.6pl cell growth and migration. 
CHAPTER 1: INTRODUCTION 


\subsection{Introduction}

\subsubsection{Cancer: A Public Health Concern in Need of Improved Therapies}

As of 2011, cancer narrowly trailed heart disease as the second leading cause of death in the United States, resulting in 575,313 deaths annually (1). The statistics aren't as grim as they once were. The overall 5 -year survival rate has increased from $49 \%$ in 1975 to $68 \%$ in 2009 (2), but there is still room for improvement. Some cancers, such as pancreatic cancer, are in dire need for improved therapies and patient outcomes; these patients have seen no change in survival during the last 30-plus years. Surgery and radiation therapy were the primary modes of cancer treatment until the 1960s, achieving a treatment success rate of $33 \%$. During the 1960 s, oncologists began administering chemotherapeutic drugs as an adjuvant to surgery and/or radiation (3) and survival rates began to rise. Historically, the bulk of anti-cancer treatments have focused on the tumor itself, but it has become more apparent that the microenvironment, including immune, fibroblast, and endothelial cells, plays a large role in the progression of cancer (4). In fact, Hanahan and Weinberg added "sustained angiogenesis" as one of their original "Hallmarks of Cancer" in 2000 (5) and a follow-up paper in 2011 included immune cells in the next generation hallmarks (6). Therefore, re-thinking the traditional tumor-only treatment paradigm and shifting to one that includes the microenvironment may provide needed improvement to the 5-year survival rate.

\subsubsection{Cancer is Dependent on Neovascularization}


Almost 75 years ago, in 1939, Ide et al. observed that rabbit epithelioma tumors grown in a transparent ear chamber were accompanied by the generation of neovessels (7). Many studies reporting similar observations followed (8-10), but it wasn't until the early 1970s that Judah Folkman published a pair of papers laying the groundwork for the hypothesis that inhibition of neovessels could be used to treat cancer $(11,12)$. Folkman's anti-angiogenesis hypothesis was formed from several key findings. The first being that without neovascularization, solid tumors stopped growing once they reached a size of 2 to $3 \mathrm{~mm}$ in diameter. Tumors placed in avascular sites such as the anterior chamber of the eye do not grow past 2 to $3 \mathrm{~mm}$ even after one year of implantation. However, if the same tumors are removed from the avascular site and placed in a site of high vascularity, muscle, rapid neovascularization and tumor growth result (13). The next finding was the discovery and isolation of a mitogenic factor from human and animal tumors that induced rapid neovascularization in vivo. This factor was appropriately called "TumorAngiogenesis Factor" (TAF) (14). Folkman reasoned that if TAF could be blocked early in the development of tumors, when the tumor is small (less than 2 to $3 \mathrm{~mm}$ ) and avascular, the tumor would arrest and in effect be controlled, thus, the beginning of the concept of vessel blockade as a viable therapeutic strategy against cancer.

\subsubsection{Angiogenesis: Physiologic and Pathologic}

Angiogenesis, as first coined by Phillipe Shubik (15), is the growth of new blood vessels from previously existing vessels. This process occurs physiologically during embryonic development, wound healing, collateral formation after myocardial infarction, and menstruation. In a developing embryo, a vascular plexis is formed and vessels sprout 
from this plexis to vascularize developing organs (16). This process is tightly controlled - vessels are induced for a brief period of time, then are inhibited to a quiescent state. Control of this process is achieved through a delicate balance of pro and anti-angiogenic factors that quickly return vessels to a quiescent state after angiogenesis is initiated. In an adult human in sites that do not require continued angiogenesis, endothelial cell turnover is measured in years (16).

In pathologic angiogenesis as in ocular diseases and cancer, the scale is greatly tipped toward pro-angiogenic factors and new vessels are formed rapidly and continue unchecked without restraint from anti-angiogenic factors. Tumors have been described as "wounds that do not heal" in a review by Harold Dvorak (17). Unregulated growth results in vessels that are fundamentally different from vessel formed under physiologic conditions. Tumor vessels are often larger in diameter, tortuous and leaky (16). Further, newly formed tumor vessels lack significant smooth muscle cell and mature pericyte investment (18). These abnormalities lead to spatial and temporal heterogeneous blood flow, resulting in areas of hypoxia. Resistance to chemo and radiotherapies is thought to arise due to uncontrolled and rapid tumor vessel development. Ineffective blood flow limits the delivery of chemotherapeutics, while areas of hypoxia limit the efficacy of both chemo and radiotherapies (18). Since tumors are dependent upon neovascularization for their growth, a vast amount of research has been conducted to understand the factors involved in angiogenesis with the goal of designing anti-vascular strategies to augment existing therapies.

\subsubsection{Angiogenic Factors}




\subsection{Vascular Endothelial Growth Factor (VEGF)}

VEGF, the most well-known and characterized angiogenic factor, was discovered in 1983 and originally called vascular permeability factor (19). Since its discovery, several families of VEGF have been identified, including VEGF-A, VEGF-B, VEGF-C, and VEGF-D. VEGF-A, the most studied family member , is often referred to as simply VEGF; this will be the case for the remainder of this text. Six isoforms of a varying number of amino acids $(121,145,165,183,189$, and 206) are generated by alternative VEGF splicing (20). VEGF 165 is the most abundantly expressed isoform (20). VEGF is normally expressed at low levels by a variety of human and animal tissues, but increases in expression are observed when angiogenesis is required. A wide variety of tumors highly express VEGF (21). VEGF primarily binds three receptors, Flt-1 (VEGFR1) (22), KDR/ Flk-1 (VEGFR2) (23), and Flt-4 (VEGFR3) (24). In the tumor context, VEGF is primarily expressed and secreted by tumor cells, while the VEGFRs are generally expressed in endothelial cells. VEGFR1 has the highest affinity for VEGF, but activation of this receptor doesn't result in an increase in proliferation or migration (25). Therefore, it is theorized that VEGFR1 modulates VEGFR2 signaling by serving as a "VEGF-trap" (26). VEGFR2 is expressed in tumor endothelial cells (27). VEGFR2 binds VEGF with less affinity than VEGFR1, but activation leads to increases in endothelial cell proliferation and migration (25) and as such, VEGFR2 is the target of many anti-vascular therapies to be discussed later in the text. VEGFR3 is primarily expressed by adult lymphatic endothelial cells and binds VEGF-C and VEGF-D, but not VEGF (28). 
As its original name, vascular permeability factor, suggests, VEGF is associated with vascular permeability. VEGF causes permeability by inducing fenestrations (29) through disorganization of endothelial cell junction proteins (30). In addition to increasing permeability, VEGF also increases endothelial cell proliferation (31) and migration (32) in vitro. VEGF also acts as an inhibitor of apoptosis, and as such is considered an endothelial survival factor (33). These effects are not confined to in vitro observations, as VEGF expression and microvessel density (a quantitative measure of in vivo endothelial cell proliferation) are positively correlated in human resected invasive ductal breast (34) and ovarian (35) cancers. Unfortunately, the study of VEGF signaling in mouse models is not possible because even heterozygous (+/-) VEGF mutants display embryonic lethality (36).

\subsection{Fibroblast Growth Factor (FGF)}

Although VEGF is the most studied angiogenic factor, FGF was actually the first pro-angiogenic factor to be identified (37). There are at least 20 family members, but two predominant forms of FGF are the most studied in endothelial signaling - acidic/basic FGF, aFGF/bFGF, or FGF1/FGF2. Interestingly, FGF1 and FGF2 do not contain canonical secretion sequences like other growth factors. The exact mechanism by which FGF2 crosses the plasma membrane is still unknown (38). Nevertheless, FGF1 and FGF2 are secreted and bind heparin sulfate on the cell surface and extracellular matrix, creating depots ready to be released by heparinases (39). FGFs bind either of 4 receptors termed FGFR1 through FGFR4. Both FGF1 and FGF2 can bind any of the 4 receptors with high affinity to activate downstream signaling (40). 
FGF2 stimulates endothelial cell proliferation $(41,42)$ and migration $(41,43)$ in vitro. An additional effect of FGF2 stimulation is increased production of urokinase-type plasminogen activator (u-PA). When grown in a 3-D collagen matrix and stimulated with FGF2, endothelial cells upregulate uPA expression and form capillary-like tubes (44). As with VEGF, FGF is positively correlated to MVD in human parathyroid (45) and gastric (46) tumors. Spontaneously generated PyVT+ tumors in an FGF2 (-/-) mouse background displayed impaired tumor growth and microvessel density (47). Interestingly, in vivo FGF2 (-/-) retina pigment epithelium tumors did not exhibit a difference in microvessel density (48), suggesting that FGF2 contribution to tumor vessel growth might be cell type and model system dependent.

\subsection{Other angiogenic factors}

The VEGF and FGF families of angiogenic factors have been extensively studied, but there are other factors that are though to play a role in tumor progression and vessel development. Platelet-derived growth factors (PDGFs) share a considerable portion of sequence with VEGF, although their expression is much more promiscuous. PDGF and its receptors are expressed in fibroblasts, smooth muscle cells/pericytes, endothelial cells and neural cells (49). PDGF molecules can form homo or hetero-dimers named PDGFAA, PDGF-BB, or PDGF-AB, constituted from either A or B chains. PDGF receptors also function as homo or heterodimers composed of $\alpha$ and $\beta$ chains. Evidence of the PDGF family's role in angiogenesis exists both in vitro and in vivo. Endothelial cells stimulated with PDGF-BB form sprouts in an in vitro aortic ring angiogenic assay (50). In vivo evidence of PDGF's importance in angiogenesis lies in observations that PDGF- 
B-deficient mouse embryos were deficient in pericyte coverage and had extensive hemorrhaging (51), observations consistent with tumor vessels. Other important angiogenic signaling pathways include angiopoetins and tie receptors, transforming growth factor- $\beta$, tumor necrosis factor- $\alpha$, and many others (52).

\subsubsection{Anti-vascular therapy}

In the early 1970s, Judah Folkman set forth a hypothesis that set the stage for the development anti-vascular therapies as a cancer therapeutic. He stated that since tumors are dependent upon the growth of new vessels to progress beyond a 2-3 mm diameter, limiting these vessels should, in theory, limit tumor growth. The preclinical research phase of these theoretical therapies was long - 33 years elapsed before the first angiogenesis inhibitor was approved by the Food and Drug Administration (FDA). As the preeminent thought leader in anti-vascular therapies, Folkman also suggested a classification of two modes of therapy - direct and indirect anti-vascular therapies (53). Direct therapies target an endothelial cellular process important to the development of tumors, including proliferation and migration, while indirect therapies are designed to target a specific tumor or endothelial cell factor. Examples of direct therapies include endostatins, matrix metalloprotease (MMP) inhibitors, and thalidomide. Antibodies, either acting as a decoy or functionally blocking receptors, and small molecule inhibitors of specific factors such as VEGF are examples of indirect therapies. Much of the early work on anti-vascular work focused on direct inhibitors such as angiostatin $(54,55)$ and endostatin $(56)$. These studies reported complete tumor regression after systemic administration of either inhibitor and, as such, excitement continued to build. A shift towards indirect therapies fol- 
lowed and now the majority of anti-vascular therapies are indirect therapies. The focus on indirect therapies could have risen from the belief that broad spectrum (direct) inhibitors increase toxicity, while more focused (indirect) therapies are less toxic (57).

\subsubsection{Anti-VEGF therapies}

VEGF is the most studied and well-characterized tumor angiogenic factor; therefore, the overwhelming majority of anti-vascular therapies have been developed against

it. In fact, every FDA approved anti-vascular therapy between 2004 and 2012 targeted the VEGF pathway (58). Anti-VEGF therapies are comprised of 2 types of drugs, antibodies against VEGF or VEGFR2 and small molecule inhibitors of VEGFR2.

\subsection{Monoclonal antibodies against VEGF or VEGFR2}

The leader in this class, bevicizumab, is a monoclonal antibody against soluble VEGF, effectively acting as a "VEGF" trap (59). Binding soluble VEGF produced by the tumor or other tumor infiltrating cells in the stroma leaves less to interact and activate VEGFR2 and its angiogenic downstream signaling. In preclinical studies, bevicizumab demonstrated tumor growth reduction ranging from $25 \%$ to $95 \%$ in a wide variety of tumor xenografts including, colon, ovarian, prostate, breast and pancreatic (60). These positive preclinical results pushed investigations into the clinic. Undoubtedly, the largest success for the theory of anti-angiogenic therapy came in 2004 when bevicizumab (trade name Avastin ${ }^{\circledR}$ ) was FDA approved for use in combination with standard chemotherapy (irinotecan, fluoruracil, and leucovorin (IFL)) in metastatic colon cancer (FDA News Release, February 2004). Bevicizumab combined with IFL prolonged progression-free sur- 
vival from 6.2 to 10.6 months and overall survival from 15.6 to 20.3 months (61). Although gains in survival were measured in months, this marked the first anti-angiogenic drug approval at that time and paved the way for approval of bevicizumab in a variety of indications including non-small cell lung cancer (NSCLC), glioblastoma, renal cell carcinoma, and metastatic breast cancer (cancer.gov). Similar to metastatic colon cancer, survival gains in each approved indication ranged from 2 to 5 months (62). Another common theme in these studies was the presence of high-grade adverse events. Unfortunately, the FDA approval for bevicizumab for treatment of breast cancer was revoked in 2011, citing that upon further review, there is no survival benefit that justifies the potentially life-threatening risks associated with treatment (FDA News Release, November 2011).

Other monoclonal antibodies have been developed for use in anti-angiogenic therapy, although they were not ultimately approved by the FDA. IMC-1C11 is a chimeric VEGFR2 binding antibody that blocks interaction with other VEGFRs, preventing endothelial signaling. A Phase I trial showed that IMC-1C11 was well tolerated and toxicity was minimal. There were no high-grade adverse events, only minor low-grade bleeding events that were quickly resolved without intervention. Although not the direct objective of a Phase I study, anti-tumor efficacy was investigated and $25 \%$ of the patients had stable disease for at least 4 weeks and 1 patient had stable disease up to 28 weeks after the initiation of treatment (63). These results were promising - less toxicity and some signs of stabilization of disease - but no further Phase II or III trials were conducted.

\subsection{Small molecule inhibitors of VEGF or VEGFR}


The successful targeting of the Bcr-Abl fusion kinase responsible for chronic myelogeneous leukemia (CML) (64), paved the way for other small molecule inhibitors in the treatment of a variety of diseases. Sunitinib, an inhibitor that targets all three VEGFRs as well as a host of other tyrosine kinases like both PDGFRs, Kit, Flt-3 and colony-stimulating factor-1 receptor (CSF-1R) (65), is generally considered an antivascular agent. Currently, it's approved as a $1{ }^{\text {st }}$ line monotherapy in renal cell carcinoma and as a $2^{\text {nd }}$ line monotherapy in the treatment of gastrointenstinal stromal tumors (GIST) (62). In the successful clinical trials that lead to their FDA approval, sunitinib showed familiar progression-free survival benefits in GIST patients (from 1.5 to 6 months) (66) and metastatic renal carcinoma patients (from 5 to 11 months)(67). Additional clinical trials were initiated in pancreatic neuroendocrine tumors (PNET), NSCLC, breast cancer, colorectal cancer, and hepatocellular carcinoma (HCC), but approval was not granted for these indications (62).

Sorafenib, another small molecule inhibitor, has a very similar inhibition profile compared to sunitinib. Studies have shown activity against all three VEGFRs, PDGFR- $\beta$, Flt-3, Kit, and Raf (68). Currently there are two FDA approvals, $1^{\text {st }}$ line treatment of renal cell carcinoma and $2^{\text {nd }}$ line treatment of HCC (62). Modest increases in HCC patient overall survival (8 to 11 months) lead to approval (69). In renal cell carcinoma, the progression-free survival benefit increased 3 months (from 3 to 6 months) (70). Clinical trials were also initiated in NSCLC and thyroid cancer, but approval was not given (62).

Recently, a third small molecule inhibitor of all three VEGFRs, both PDGFRs and FGFR1 and 3, pazopanib (71), has been FDA approved as a $1^{\text {st }}$ line treatment for patients 
with renal cell carcinoma (62). Clinical trial data show a 5-month increase in progression-free survival from 4 to just 9 months (72). Although increases in progression-free survival were similar between pazopanib and sunitinib, the safety profile and quality of life profiles were more favorable in patients treated with pazopanib (73). Like the other VEGFR small molecule inhibitors, unsuccessful clinical trails were initiated in a number of other indications, including ovarian, breast, NSCLC (62).

\subsubsection{Other angiogenic factor inhibitors}

As there are a plethora of pro-factors involved in vascular development, other therapies against non-VEGF proteins have been developed. These include brivanib, cediranib, vandetanib, and axitinib (62). Although each is responsible for at least 3 clinical trials, none are currently approved by the FDA as anti-vascular agents.

\subsubsection{Modest survival resulting from resistance to anti-vascular therapy}

Although an overwhelming amount of preclinical data showed almost complete tumor and tumor vessel regression, clinical trial results were not as promising, independent of the mode of inhibition. The general course of response includes an initial stasis or shrinkage of the tumor, followed by a period where the tumor begins to progress, terminating the progression-free survival end point and ultimately reducing overall patient survival. These observations are especially disconcerting because the widely accepted hypothesis set forth by Judah Folkman states that tumors are dependent on their vessels and that anti-vascular therapy should eliminate or reduce tumors back to their avascular size. In anti-cancer therapies, two types of resistance exist - innate and acquired (74). Typi- 
cally, these types of resistance arise from the genetic and epigenetic instability of cancer cells. Endothelial cells are generally regarded as genetically and epigenetically stable. However, many pro-angiogenic factors are secreted by cancer cells in a paracrine fashion. In fact, resistance to anti-vascular therapies may not result from endothelial cells, rather the surrounding cancer cells that are driving vessel growth because vessel growth is necessary for tumor progression. Further introspection reveals many preclinical and clinical examples of innate and acquired resistance.

\subsubsection{Innate Resistance}

In the clinical trials of bevicizumab, sunitinib, and sorafenib, there was always a small subset of patients who exhibited progressive disease and ultimately never responded $(67,70)$. Responses are typically assessed only at the end of each cycle, therefore refractoriness from the onset of the trial cannot be confirmed; however, it is very plausible that patients exhibited resistance from the beginning. The existence of phenotypic and functional heterogeneity of cells within the same tumor is well-known in cancer research (75). If intratumoral heterogeneity exists, it follows that each patient's tumor is unique in its cell composition, namely activating mutations and signaling. These observations have driven the idea of personalized medicine in cancer treatment. Innate resistance to anti-vascular drugs could also be explained by this phenomenon, as individual tumors express and are dependent on different angiogenic factors (76). For example, one tumor could predominantly express VEGF, while another expresses FGF2, and yet another IL8; the first would be responsive, while the second and third tumor would be innately resistant to anti-VEGF agents. 
A second contributor to innate resistance could be time at which clinical trials are initiated. Unfortunately, in most clinical trials, patients are at the end of their treatment options and their enrollment into a trial is dependent on their refractoriness to standard of care treatments. At this point, their tumors have been present for months or years and are typically at an advanced stage. This is in contrast to typical preclinical anti-vascular agent studies that examine response in tumor xenograft models in the incipient stages of tumor development. Clinically, the course of angiogenic factors is known to change as the disease progresses. Serum analysis of melanoma patients revealed that in the early stage of disease (stage I), VEGF dominates as the lone angiogenic factor present, while in stages II and III, FGF2 and IL8 are extremely overexpressed, while VEGF is slightly upregulated (relative to stage I) (77). One can hypothesize that patients with advanced disease - when patients are often treated with anti-vascular agents - have tumors with redundant, activated angiogenic signaling pathways and thus exhibit innate resistance to single factor (anti-VEGF) therapy.

Another consequence of the timing of clinical trials is that tumors and associated vessels are well established when patients begin treatment. Preclinical mouse studies suggest that vessel maturity is an important factor in determining susceptibility to VEGF therapy. Mice subcutaneously injected with VEGF-A-expressing adenovirus (Ad-VEGFA) develop neovasculature that includes all blood vessel subtypes observed in human tumors - mother vessels, glomeruloid microvascular proliferations, vascular malformations, feeding arteries, and draining veins (78). Injection of a VEGF-trap agent Aflibercept either before or a few days after injection of Ad-VEGF-A reduces neovessel development. 
In contrast, if treatment with Aflibercept is delayed until late-forming vessel subtypes (vascular malformations, feeding arteries, and draining veins) have developed, no regression is observed (78). Variable vessel subtype susceptibility to anti-VEGF treatment could have important implications in understanding why VEGF treatments have disappointed in the clinic.

\subsubsection{Acquired resistance}

Some patients undergoing anti-vascular therapy initially respond, with either stable or regressing tumors, but resistance eventually develops and the patient succumbs to their disease (79) As already stated, tumor cells are genetically and epigenetically unstable. This instability enables tumors to rapidly select drug resistant cells that survive treatment due to some survival advantage conferred by one or many of the thousands of possible mutations (80). Examples of this selection can be found both in mice and humans undergoing anti-vascular therapy. In a RIP-Tag2 pancreatic islet tumor model, animals treated with the VEGFR2 blocking monoclonal antibody DC101 initially respond, both tumor burden and vessel density (81). However, after just 10 days of treatment, tumors relapse, vessel density increases and tumors grow unabated. Further examination of tumors at the point of relapse revealed that mRNA and protein levels of the pro-angiogenic factors VEGF-A, several FGF family members, and angiopoeitin 1 were upregulated compared to control treated tumors. Interestingly, addition of an FGF-trap to the treatment schedule after the tenth day prevented this relapse from occurring (81). In an extension of this work, brivanib, a dual VEGFR/FGFR inhibitor was used successfully as a $1^{\text {st }}$ or $2^{\text {nd }}$ line therapy after failure of DC101 or sorafenib in RIP-Tag2 mice (82). The search for pre- 
dictive biomarkers of response to bevicizumab has coincidentally provided information about angiogenic factors that are upregulated in response to anti-VEGF therapy (83). FGF2 plasma concentration increased after the second cycle of bevicizumab treatment in lung cancer patients (84). Plasma FGF2 concentration was increased just before and at the time of progression in colorectal patients treated with bevicizumab (85). Additionally, plasma PDGF-BB and stromal cell-derived factor 1 (SDF1) concentrations were increased at the time of progression in colorectal cancer patients (85).

\subsubsection{Discovery of additional VEGF-independent angiogenic factors}

These data strongly indicate that targeting the vasculature, once considered an attractive strategy on the theory that endothelial cells are genetically stable, is prone to both innate and acquired resistance. This is especially true when targeting one pathway, VEGF, as has historically been the case. Therefore, the identification of factors that signal in compensatory or redundant, VEGF-independent pathways could direct the development of anti-vascular strategies that improve cancer patient outcomes.

\subsubsection{Approaches to identify VEGF-independent factors}

The development of sophisticated genomic and proteomic screening methods in the past few decades has greatly furthered our understanding of the molecular origins of disease. In fact, somewhat ironically, investigators have recognized that genomics approaches such as microarray expression analyses have the potential to generate too much data and that care must be taken so that essential findings are not diluted by the massive production of often-unannotated data (86-88). Genomics approaches include genome 
sequencing and gene expression microarray analysis. Proteomics approaches include protein microarrays, two-dimensional protein agarose gel electrophoresis (2D-PAGE), and phage display.

\subsection{Genomics methods}

\subsection{Comparative genome sequencing}

The development of genome sequencing technologies greatly advanced the knowledge we have about human biology and disease. The Human Genome Project (HGP), launched in 1990 and completed in 2011, provided a "genetic instruction book" to guide future research into evolution, physiology, and human development (89). Since the completion of the human genome, a large number of other organisms have been successfully sequenced. Non-human genome data is primarily used in evolution research, but can be important in discovering the origins of disease. The primary application of the HGP sequencing has been in comparisons of wild-type genomes to those of diseased patients to detect "driver mutations" (90) that are thought to cause cancer or genes that confer resistance to chemotherapeutics (91-93). This approach has identified IDH1 mutations in glioblastoma multiforme (GBM) (94). Although the IDH1 mutation has been primarily used as a prognostic marker in GBM, therapies could certainly be designed against it. Preclinical studies of an inhibitor of mutant IDH1 show treatment results in delayed growth in glioma cells (95).

Although the ability to sequence the entire genome of patients opens up a vast array of data and possibilities, it is a rather expensive genomic approach. Consequently, 
one of two methods has historically been employed: 1) sequence a large number of samples, but just a few genes or 2) sequence all coding genes in a small number of samples (96). The development of next generation sequencing has enabled the entire genome to be sequenced in a much more time and cost efficient manner. The big thrust in the past decade has been to bring the cost of sequencing patients entire genomes down. Considerable progress has been made as the cost to sequence one genome has decreased from $\$ 95,000,000$ in 2001 to $\$ 6,000$ in 2013 (NHGRI Genome Sequencing Program) However, the expertise and computational power needed to analyze these large data sets limits this method's utility in smaller laboratories. The ultimate limitation in this approach is that the endothelial cell genome is generally regarded as stable and therefore, the search for a VEGF-independent factor by comparative genome sequencing is not likely to be a fruitful one.

\subsection{Differential gene expression analysis}

As mentioned earlier, the HGP allowed the sequencing of over 20,000 genes cataloging the complete list of genes that could possibly be involved in cellular function or dysfunction. Differential expression of genes in control and disease states is used to identify key regulators of disease. The most widely method used to study differential gene expression is the microarray platform, developed in the mid-1990s (97). Early microarrays were prepared by the investigators themselves, but microarrays are now commercially available through many vendors. In this method, oligonucleotides that correspond to genes or cDNAs are spotted onto a silicon wafer, glass slide or nylon membranes (97). Next, RNA samples from disease and control tissue are amplified and la- 
beled with one of two fluorophores, typically Cy3 (green) and Cy5 (red). Labeled RNAs (typically mRNAs) are incubated on the pre-spotted chip. After hybridization, fluorescence intensity in each channel is measured at each spot. The intensity of each color channel corresponds to the expression of a particular gene. If diseased tissue mRNA is labeled green and control tissue mRNA labeled red, then a green spot would indicate that that particular gene is overexpressed in diseased tissue compared to control. A red spot indicates that a particular gene is overexpressed in the control tissue relative to the diseased tissue. Lastly, a yellow spot would indicate that a particular gene is expressed equally in each state.

Gene expression microarray analysis has produced large quantities of data that have necessitated the creation of databases to organize and archive data such as the Gene Expression Omnibus (GEO). Gene expression microarrays have identified a number of angiogenesis genes from colorectal cancer (98), prostate cancer (99), and lung cancer (100) tumor samples. Likewise, the identification of vascular genes via microarray analysis has identified tumor endothelium specific genes which may be important in tumor vessel development $(101,102)$. Knowledge of differentially expressed genes could direct the development of targeted therapies to these genes.

Microarray gene expression analysis has become popular because it is relatively low cost, easily available, and provides vast amounts of unbiased data. The last attribute could actually be a detriment. The immense amount of unbiased data that is generated from a microarray screen can be quite cumbersome to investigators. A typical screen produces anywhere from 50-200 "hits" that require additional validation and functional 
studies. The number of hits is intractable, so how does an investigator pare down the number of hits to a more manageable number? Often, investigators browse the literature and look for hits that "make sense" based on previous studies about the particular disease that is being studied. While this is a perfectly acceptable reduction method, it takes an elegantly designed, unbiased screen and biases it, essentially changing the mission of the study from an unbiased discovery screen to a narrowed discovery screen of previous reported hits. Further, it is well known that RNA expression does not correlate with protein expression, despite what the accepted linear central dogma of molecular biology "DNA --> RNA --> Protein" might suggest. In a metastudy of 1066 genes in 23 human cell lines, Gry, et al. found that the average correlation between mRNA expression and immunohistochemical protein expression was $\sim 0.3$, suggesting that mRNA hits should be interpreted with caution (103). For these reasons and since disease is ultimately the result of misregulated protein expression and/or localization, proteomics approaches are favored by our laboratory.

\subsection{Proteomics methods}

\subsection{2D-PAGE or 2D-DIGE methods}

2D-PAGE has become the most widely used proteomics method; its popularity results from the ability to separate large numbers of proteins, and ability to determine relative abundance of proteins (104). In this technique, proteins are isolated from tissue sam-

ples, usually control and diseased, and loaded in separate gels. Proteins are typically separated in the first dimension by isoelectric point. Next, sodium dodecyl sulphate 
(SDS) is added to the gel and proteins are further separated by the second dimension, molecular weight (105). Finally, protein signatures are stained by some in-gel staining method such as coomassie or silver stain and analyzed for differences between the control and diseased gels. 2D-PAGE has identified prognostic markers for colorectal cancer (106) and lung cancer (107). However, as newer proteomics methods were developed, 2D-PAGE became subject to the criticism that it is often irreproducible between the two sample gels, and too time consuming (108). In an attempt to eliminate some of the critiques of 2D-PAGE, two dimensional difference gel electrophoresis (2D-DIGE) was developed (109). In this technique, each pool of proteins is labeled with a different aminereactive fluorophore and separated on the same gel, two or more images are taken (one for each fluorophore) and then superimposed, thus eliminating the reproducibility concerns. Like 2D-PAGE, 2D-DIGE has been used to identify biomarkers of lung cancer using saliva samples (110).

While successfully used for discovery based protein identification, 2D-PAGE/DIGE suffers from one of the most fundamental difference between genomics and proteomics research - there is no way to amplify isolated protein. Therefore, 2D-PAGE/DIGE is only capable of detecting abundant proteins. While one might conclude that abundant proteins imply importance to pathophysiology, this is not always the case. Silver stained gels are capable of detecting proteins with 1,000 copies or more per cell. An analysis of protein copy number in cultured human U2OS cells revealed that highly abundant $(>1,000$ copies per cell) only account for $63 \%$ of all proteins (111). Therefore, $37 \%$ percent of all proteins are therefore undetectable by 2D-PAGE/DIGE. A final limitation is 
that membrane proteins tend to aggregate in solution and are therefore poorly represented in 2D-PAGE/DIGE analyses (112). While finding a membrane protein is not absolutely necessary in a typical proteomics discovery experiment, if a membrane protein is identified, it can be used in both therapeutic and imaging applications. To maximize the potential of proteins found through proteomics screens, our laboratory prefers to use phage display and functional proteomics methods to identify new imaging agents and biologic targets.

\subsection{Phage display}

Phage display was introduced by George Smith in 1985 (113) and since then has been widely used to study protein-protein and protein-non-protein interactions (114). Typically, a random library of peptides are fused to the pIII coat protein and displayed on the end of filamentous phage; however, a library of antibody fragments are sometimes displayed on pVIII proteins (115). These libraries are used to identify peptides that bind to either a specific target molecule or an interesting population of cells through multiple rounds of selection and amplification of binding phage clones. At the end of a screening experiment, phage clones are sequenced and peptides that bind the intended target are identified. The popularity of phage display has lead to the development of several commercially-available peptide phage display libraries. The most common libraries are sold by New England Biolabs and utilize the M13 bacteriophage. Phage display screening eliminates the limitations of other genomics and proteomics methods - allowing a description of the membrane proteome with no requirement of high protein abundance since phage clones are amplified via bacterial infection. 
Phage display screens have been completed in vitro, on cells (116) or purified protein (117), ex vivo on tumor sections using laser microdissection (118), and in vivo in mice (119) or humans (120). Of particular interest, is the concept of "vascular zip codes" pioneered by Erkki Ruoslahti through a series of in vivo phage display screens in mice (121). These studies reveal that each organ vascular bed, including tumors has a distinct set of peptides that preferentially home to it $(122,123)$. The most common peptide motif to come out of the tumor vasculature screens is Arg-Gly-Asp (RGD), providing proof-ofprinciple, as RGD is a known cell recognition signal that binds integrins $(124,125)$. Since RGD is well known, these findings represent important, but incremental advances in the knowledge of the proteomic landscape of endothelial cells. Even if these screens produced other novel peptide motifs, they do not give any information about the binding partner, unless it is previously known as in the case of RGD. Researchers are left to use a searchable algorithm called basic local alignment search tool (BLAST) to identify potential binding partners of expressed proteins. By matching the selected peptide with known ligands, one can find the binding partner receptor of that particular peptide. This process is inefficient and the possibility of identifying false positives is great, as 7-12-mer peptide sequences return many "hits". To take the utility of phage display further, Kimberly Kelly developed a functional proteomics approach for directly identifying the binding partner of selected phage.

\subsection{Functional proteomics}

In the functional proteomics method developed in the Kelly laboratory, phage are first bi-functionalized with biotin and a photoactivatable cross-linking molecule. Phage 
are allowed to bind their target cell, non-binding phage are washed away. Next, remaining phage are cross-linked to the nearest protein (target protein) by ultraviolet light. The cells are then lysed and phage-protein complexes are isolated by streptavidin-coated beads. The cross-link is dissolved by exposure to a reducing agent such as dithiothreitol (DTT). Samples are then separated by electrophoresis and unique bands are excised and sequenced by tandem mass spectroscopy. This method has identified plectin, pyruvate kinase M2, and annexin A2 as important proteins in pancreatic ductal adenocarcinoma (PDAC) (126) and therefore repesents an exciting new technology that pushes phage display past the identification of peptides useful for therapeutic targeting and imaging into the discovery of misregulated proteins in pathologic conditions.

To identify important non-VEGF mediated factors involved in tumor vessel development to guide the creation of new anti-vascular therapies, we used functional proteomics based phage display because of its aforementioned attributes. Hornerin, an S100 family member not previously known to be expressed in endothelial cells, was identified and subsequently validated in vitro and in vivo in mouse and human tissue. We show that hornerin does indeed contribute to the development of tumor vessels and tumor growth. Mechanistically, we show that hornerin contributes to endothelial cell survival when exposed to oxidative stress through NF- $\mathrm{KB}$ induced BCL2 colocalization to the mitochondria. Finally, combining hornerin therapy with anti-VEGF therapy produced additive dissipation of tumor growth and tumor vessel development, providing the rationale for the development of non-VEGF therapies to replace or augment existing anti-vascular therapies. 
CHAPTER 2: HORNERIN IDENTIFIED AS A NON-VEGF MEDIATED FACTOR THROUGH PHAGE DISPLAY BASED FUNCTIONAL PROTEOMICS 


\section{$\underline{2.1 \text { Abstract }}$}

Most current and past preclinical and clinically approved anti-vascular therapies focus on the vascular endothelial growth factor (VEGF) pathway - with good reason, as VEGF is highly expressed in most tumors. Unfortunately, targeting VEGF has been largely ineffective, as judged by poor clinical success in increasing patient survival. Survival gains are always measured in months, rather than years. Compensatory or redundant pathways could be the cause of anti-VEGF therapy ineffectiveness. Therapies directed against non-VEGF mediated proteins could hold the key to effective anti-vascular cancer therapies especially when combined with clinically relevant and FDA approved VEGF therapies. Therefore, we used in vivo phage display in mice with orthotopically implanted pancreatic ductal adenocarcinoma (PDAC) tumors combined with in vitro functional proteomics methods to identify VEGF-independent factors. After the in vivo phage display screen, 5 PTEM (pancreatic tumor endothelial marker) clones that bound human umbilical vein endothelial cells (HUVECs) treated with tumor conditioned media (TCM), but not VEGF conditioned media (VEGFCM), were identified via enzyme-linked immunosorbent assay (ELISA) and two-way ANOVA analysis. Through functional proteomics, hornerin was identified as the binding partner of the most specific TCM binding clone, PTEM 9. In vitro experiments confirmed hornerin as an important non-VEGF mediated protein that is upregulated by TCM. In resected human pancreatic ductal adenocarcinoma associated blood vessels, hornerin was highly expressed in an overwhelming majority of the cases, indicating potential clinical relevance of hornerin. Our findings identify hornerin as a non-VEGF mediated endothelial protein that should be investigated 
further to understand its mechanism of action and functional role in tumor vessel development.

\subsection{Introduction}

Tumors larger than 1-2 $\mathrm{mm}$ in diameter require the development of new vessels because these tumors are no longer able to access oxygen and nutrients simply through diffusion (127). Tumor vessel development proceeds by primarily two mechanisms, angiogenesis followed by nascent vessel survival (128). Anti-vascular therapies, which are almost always synonymous with vascular endothelial growth factor (VEGF) inhibitors, have been proposed and implemented alone or in combination with anti-cancer cell therapy $(129,130)$. Anti-tumor vessel (mostly anti-VEGF) therapies have achieved success pre-clinically, but those early promising results have not fully translated in the clinic. Often, patients experience a short-lived period of response, but tumors quickly become resistant and the patient ultimately succumbs to their cancer. Avastin (bevicizumab), a "VEGF-trap" drug, is clinically approved to treat a variety of cancers, but its FDA approval for the treatment of metastatic breast cancer was recently revoked, citing, that upon further review, there was no significant change in progression free survival (PFS) (FDA, News Release, November 2011).

One hypothesis for the lack of success could be the presence of compensatory or redundant pathways. Compensatory pathways are well established in tumor therapy research; consequently clinical trials devoted to synergistic combinations of drugs have proliferated (131-133). Combinatorial approaches involving non-redundant signaling pathways show great promise, as they have the ability to overcome acquired resistance to chemotherapy (134). A combination approach, one involving both the VEGF pathway 
and another important pathway, could provide the positive results that anti-vascular therapies should theoretically produce.

Therefore, to identify non-VEGF mediated factors involved in tumor vessel development, we used a phage display functional proteomics approach, combining an in vivo phage screen in tumor bearing animals and in vitro validation and binding partner identification (126). Hornerin, which was previously reported to be expressed in skin (135), was identified from our in vivo phage display based functional proteomic screen and further validated as a non-VEGF regulated protein in human umbilical vein endothelial cells (HUVECs). While confirming hornerin expression in tumor-associated endothelial cells in resected human pancreatic ductal adenocarcinoma (PDAC) samples, it was discovered that hornerin is also expressed in PDAC. Further exploration identified a myriad of other tumor types that express hornerin, including renal cell carcinoma (RCC), prostate adenocarcinoma and PDAC.

\subsection{Results and Discussion}

\subsubsection{Selected phage clones are specific for tumor vasculature in vivo}

In vivo phage display has been successfully used to characterize and describe the differences between vascular beds in a variety of organs and tumors (121-123). An in vivo phage screen is the preferred screening method in this investigation, because it ensures that the phage clones are pharmacologically suitable and that the binding target is present in vivo. Additionally, when the phage library is injected intravenously, the entirety of the animal's vasculature is used to "subtract" non-target binding clones, thereby 
increasing the stringency of the screen. If clones survive multiple rounds of highly stringent selection, there is a greater chance tumor vasculature specific clones will be isolated.

An in vivo phage display screen was completed in mice bearing orthotopically implanted pancreatic tumors. Phage were allowed to circulate for 8-10 minutes to limit phage extravasation. After 3 rounds of selection, 30 phage clones that we termed pancreatic tumor endothelial markers (PTEM) were sequenced (Figure 2.1A). To assess the in vivo selectivity of the phage pool after the three rounds of selection, we injected, via tail vein, a fluorescently labeled pool of phage clones with a common consensus motif, PRH, and performed immunofluorescent analysis, similar to a protocol described by Kelly, et al. (117). Figure 2.1B is a representative image of FITC-labeled phage clones in an orthotopic pancreatic tumor. The "PRH" pool (PTEM 12/17 and 16) bound 73.4\% (4.1-fold over normal pancreas vessels) of PECAM1-positive tumor vessels (Figure 2.1B, lower). Importantly, FITC-phage were bound to the luminal surface even after significant washing of the vessels via saline perfusion and did not extravasate deeper into the tumor. Thus, these phage are selective for tumor associated vessels. This finding instilled confidence in our in vivo phage screen methodologies and prompted us to further evaluate and validate the phage clones.

\subsubsection{Selected phage clones are specific for TCM treated HUVECs}

As VEGF is highly abundant in the tumor milieu, we decided to further segregate clones based on their ability to bind to VEGF treated HUVECs versus TCM treated HU- 


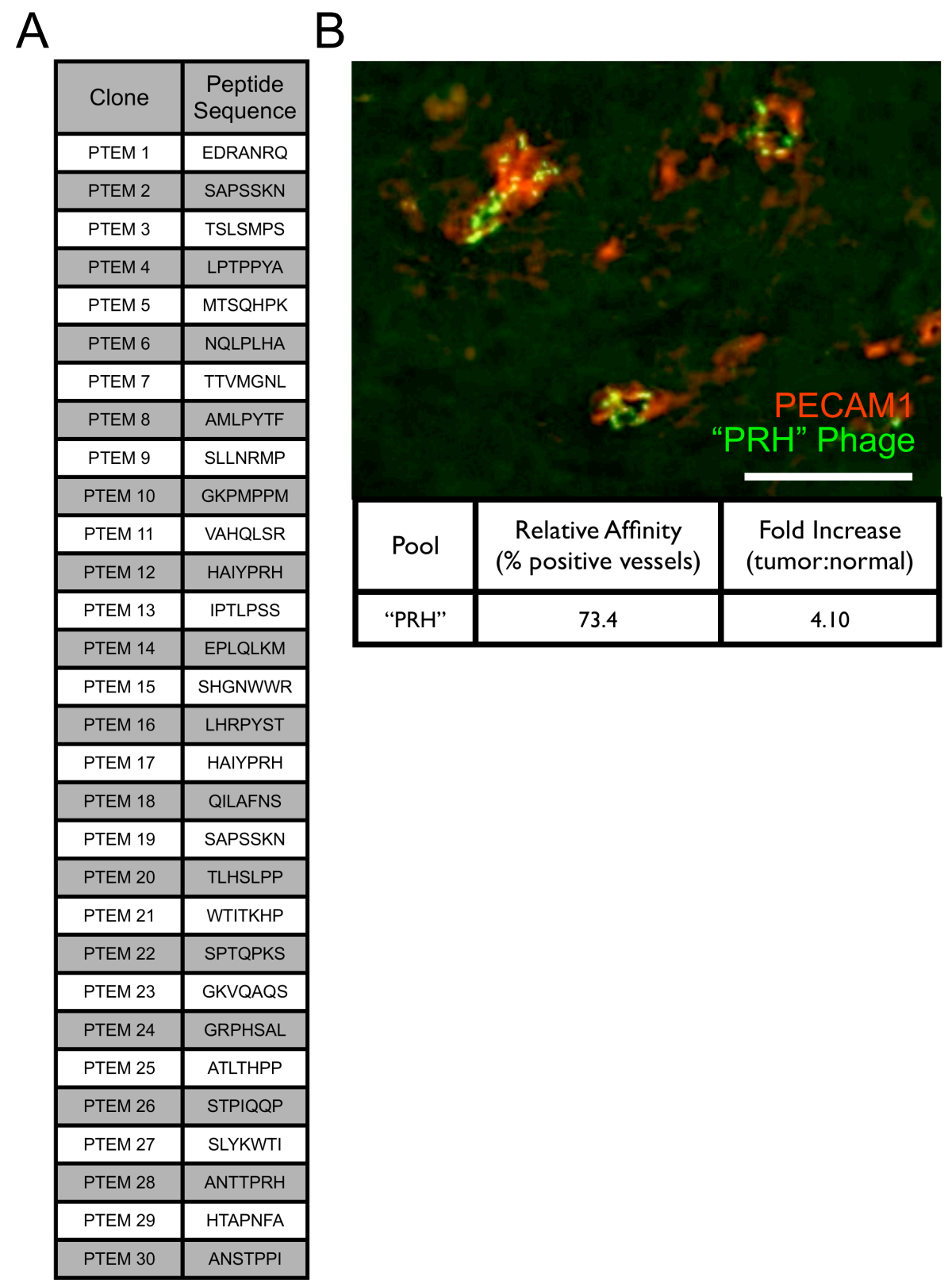

Figure 2.1 Selected phage clones are specific for tumor vasculature in vivo. A. The amino acid sequences of 30 selected phage clones are shown. B. Orthotopic pancreatic tumor section showing colocalization of FITC-labeled phage pool containing clones with the "PRH" motif with PECAM1-positive vessels. Relative affinity and fold increase over normal (adjacent, unaffected pancreas vessels) of the "PRH" pool were quantified below. 


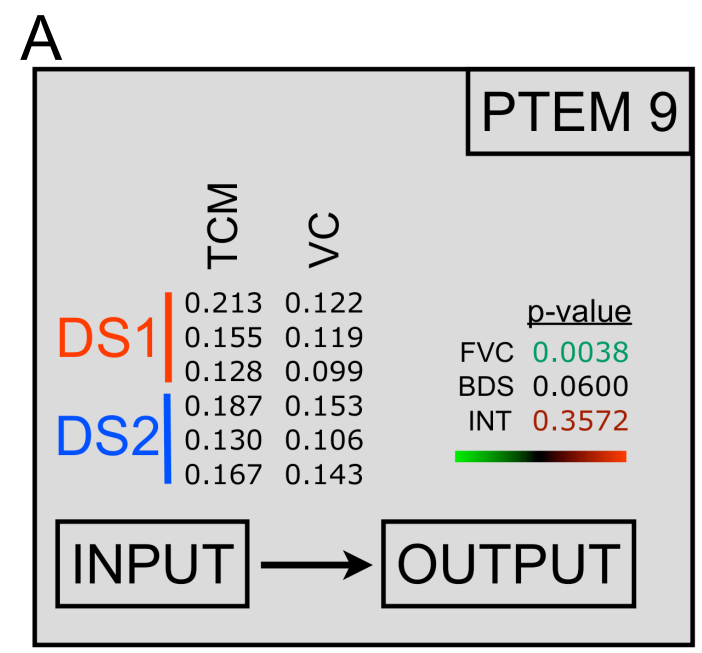

B

TCM vs. VC VEGFCM vs. VC

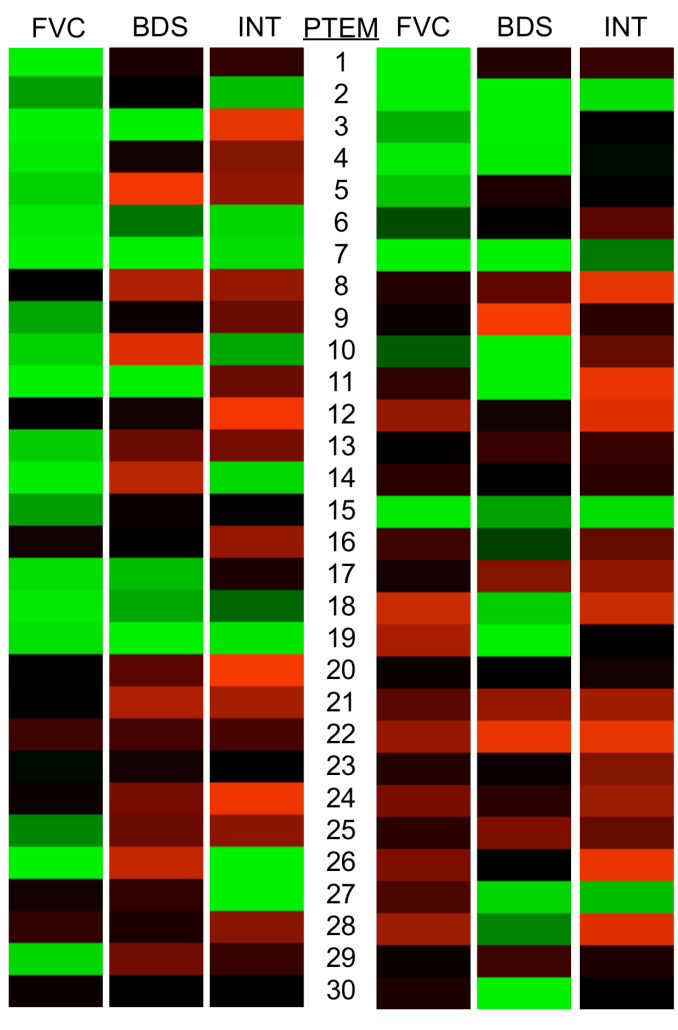

Figure 2.2 Two-way ANOVA analysis identifies TCM specifically upregulated clones. A. ELISA data from PTEM 9 is organized in matrix format, forming the input to two-way ANOVA analysis. Two independent experiments ( 3 replicates in each) were analyzed. Analysis yields 3 probabilities or p-values: fold-over vehicle control (FVC), between data sets (BDS), and an interaction (INT). B. p-values for each PTEM clone shown as heatmap for each comparison, TCM versus VC and VEGFCM versus VC. Heatmap legend is located below. 
VECs. HUVECs were used for two reasons: 1) as they are of human origin, we wanted to ensure cross-reactivity of the peptide for human and mouse proteins and 2) cultured cells provide the ample source of targets, which is essential for identifying the binding partner of the phage clones. Therefore, we performed ELISA experiments on HUVECs treated with VEGF conditioned media (VEGFCM at B-max concentration (136)), TCM, or vehicle control (VC) media. The results of two phage based ELISAs on TCM, VEGFCM, or VC treated HUVECs were analyzed by 2-way ANOVA statistical analysis to find clones that consistently bound HUVECs treated with TCM, but not VC or VEGFCM (Figure 2.2A and B). The 2 independent variables used in this analysis were type of conditioned media and the data sets collected from different experiments. Therefore, to be validated as a TCM binder, each clone must be significantly different between TCM and VC, but not VEGFCM and VC. It must also be not significantly different between data sets (experiments) or a third computed interaction term. If phage are significantly different for either conditioned media, but not any other variable, they are validated as binding to both TCM and VEGFCM. Finally, if any of these criteria are not met, the phage are not counted as TCM or VEGF binders.

The clones were grouped and organized via Venn diagram as shown in Figure 2.3A. The largest number of clones significantly bound TCM, while just 2 bound HUVECs treated with both TCM and VEGFCM. The presence of phage clones binding to HUVECS treated with TCM and VEGFCM was expected as VEGF is a known potent angiogenic stimulator present in tumors (137) and present in large abundance in our TCM. The PRH family did not survive the analysis, suggesting that either it does not 
B
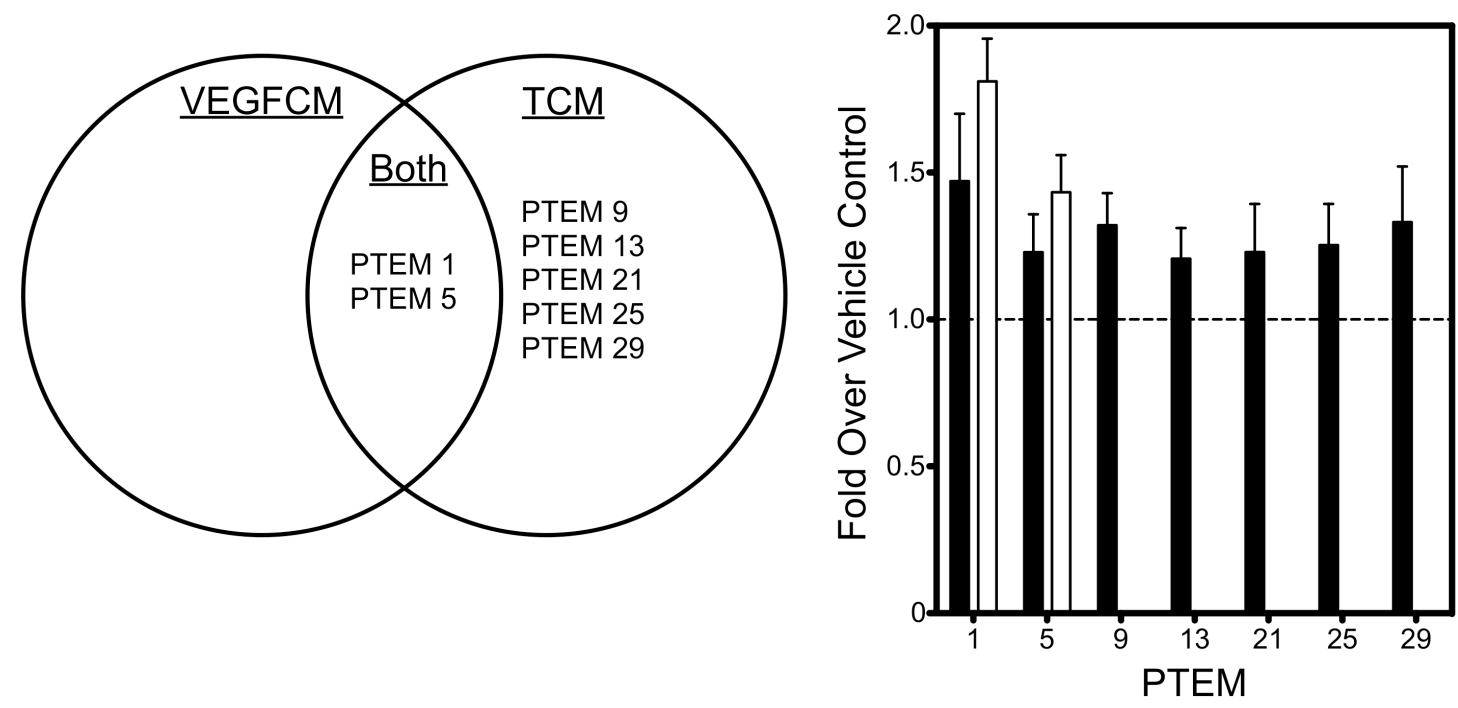

Figure 2.3 Two-way ANOVA analysis identifies TCM specifically upregulated clones. A. After phage-based ELISA and 2-way ANOVA analysis, phage clones were grouped and displayed via Venn diagram. If a phage clone is in the "Both" category, it significantly bound ( $\mathrm{p}<0.01)$ both TCM and VEGFCM treated HUVECs. B. Quantification of the specificity (Fold Over Vehicle Control) for each clone in the Venn diagram. Solid bars represent TCM; open bars represent VEGFCM. Dotted line represents a fold value of 1, the point where there is no specificity for TCM or VEGFCM over VC. Data represented as mean \pm s.e.m of 2 independent experiments. 
cross-react with a human target or the target is not present in human cells. Only 7 out of 30 phage clones were represented in the Venn diagram. We believe this is due to especially stringent conditions requiring cross-species reactivity (mouse and human) and significance in 2 replicate experiments analyzed by 2-way ANOVA analysis. The specificities of clones represented in the Venn diagram are shown in Figure 2.3B. PTEM 9 demonstrated the highest average specificity of the TCM clones, thus it was selected for binding partner identification via phage-based functional proteomics (126).

\subsubsection{Hornerin identified as the binding partner of PTEM 9}

After in vitro validation, we used PTEM 9 to identify its binding partner using previously published functional proteomics methods (126). Briefly, phage were covalently modified with a photoactivatable crosslinker and biotin. Functionalized PTEM 9 was incubated with non-permeabilized, intact HUVECs. After washing, the crosslinker was activated via exposure to ultraviolet light and the cells were lysed. Phage-protein complexes were isolated using streptavidin coated magnetic beads and phage-bound proteins were released by reducing the crosslink with dithiothreitol (DTT). The resulting lysate was run on a $4-15 \%$ gel and silver stained. The lysate from PTEM 9 was compared to a negative control KE-phage, which is M13 phage without displayed peptides (NEB) and a unique band was excised (arrow, Figure 2.4A), subjected to tryptic digest and MS/MS analysis for protein identification. Analysis of the MS/MS fragments revealed hornerin as the candidate protein; 6 unique tryptic digest fragments were identified resulting in a percent coverage of 3.5\% (Figure 2.4B), coverage similar to what has 
been previously published $(116,126)$. To confirm that hornerin was the identified protein, we immunoblotted using an anti-hornerin antibody on control and PTEM 9 lysates and found a unique band in the PTEM 9 lane (Figure 2.4C). As hornerin was not previously reported in endothelial cells or involved in tumor angiogenesis, we sought to validate this in vivo, in vitro, and in human patient samples.

\subsubsection{Hornerin is upregulated in HUVECs in response to TCM but not VEGFCM}

Since hornerin was identified through functional proteomics, it must be present on the cell surface. To date, there is no record of its presence on any cell surface. Therefore, we validated that cell surface hornerin expression was induced by TCM, but not VEGFCM or VC via immunofluorescence on fixed, non-permeabilized cells. Hornerin expression was observed in TCM treated cells but non-detectable in cells treated with VEGFCM (Figure 2.5). Cells were counterstained by fluorescently labeled wheat germ agglutinin (WGA), a lectin that binds cell membranes. To further confirm TCM specific expression, lysates of HUVECs treated with TCM, VEGFCM and VC media were subjected to western blot (Figure 2.6A). In TCM treated HUVECs, hornerin expression was 19.3-fold increased over VC, while treatment with VEGFCM resulted in no significant change in hornerin expression relative to $\mathrm{VC}$ (Figure 2.6B). The combination of the phage-based ELISA, western blot, and immunofluorescent data strongly indicates that hornerin is upregulated in response to TCM but not in response to VC or VEGFCM. 


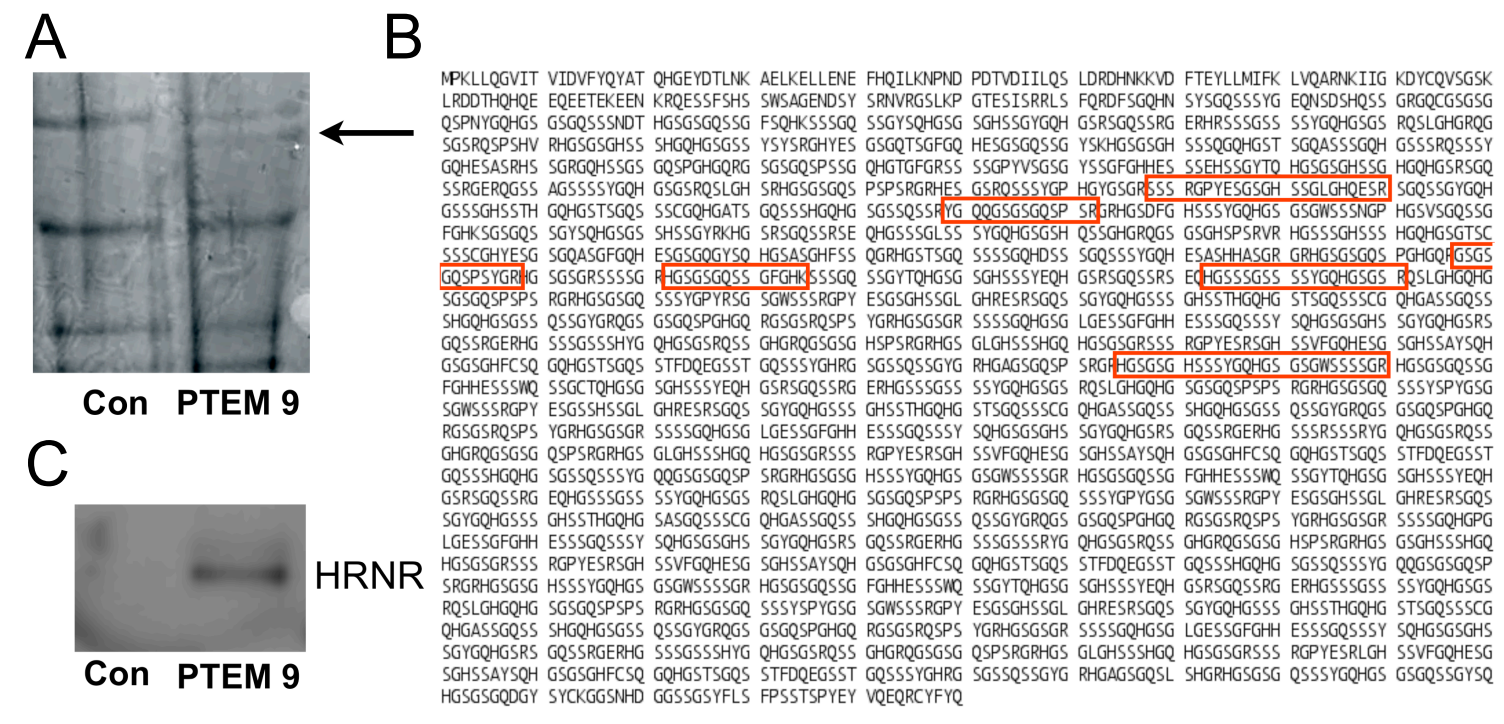

Figure 2.4 Hornerin identified as the binding partner of PTEM 9. A. Silver stained gel of control KE-phage (Con) and PTEM 9 lysates. The unique band highlighted with an arrow was excised and sent for sequence analysis by MS/MS. B. Peptide coverage map from MS/MS sequencing of PTEM 9 lysate. Peptides recovered from MS analysis are boxed in red. C. Anti-hornerin western blot of Con and PTEM 9 lysates confirming the presence of hornerin in PTEM 9 lane only. 


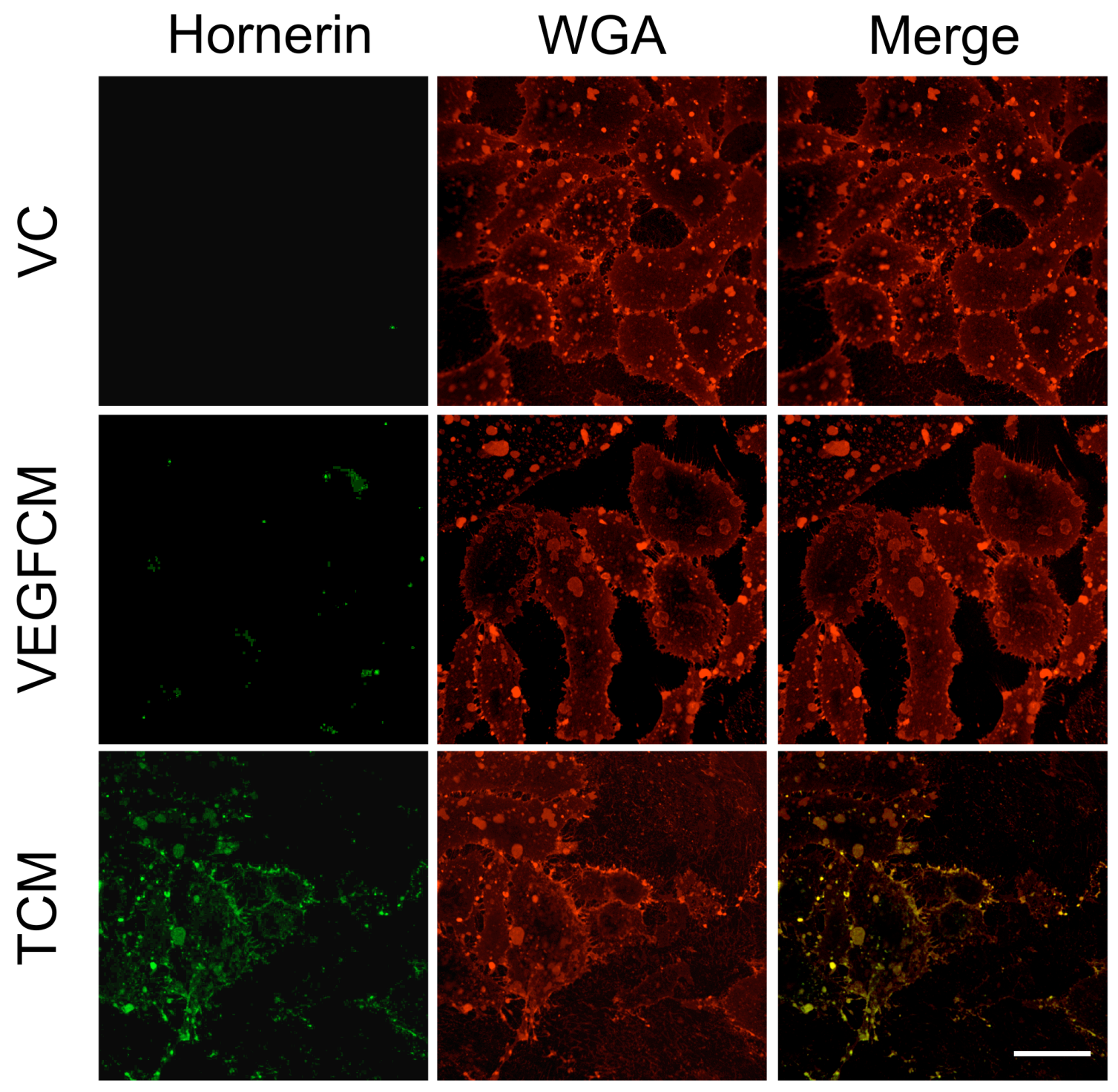

Figure 2.5 Hornerin is present on the cell surface of TCM treated HUVECs. Antihornerin immunofluorescence on intact, non-permeabilized HUVECs. Images were captured by confocal microscopy. Wheat-germ agglutinin (WGA) was used as a cell counterstain. Scale Bar: $50 \mu \mathrm{m}$ 

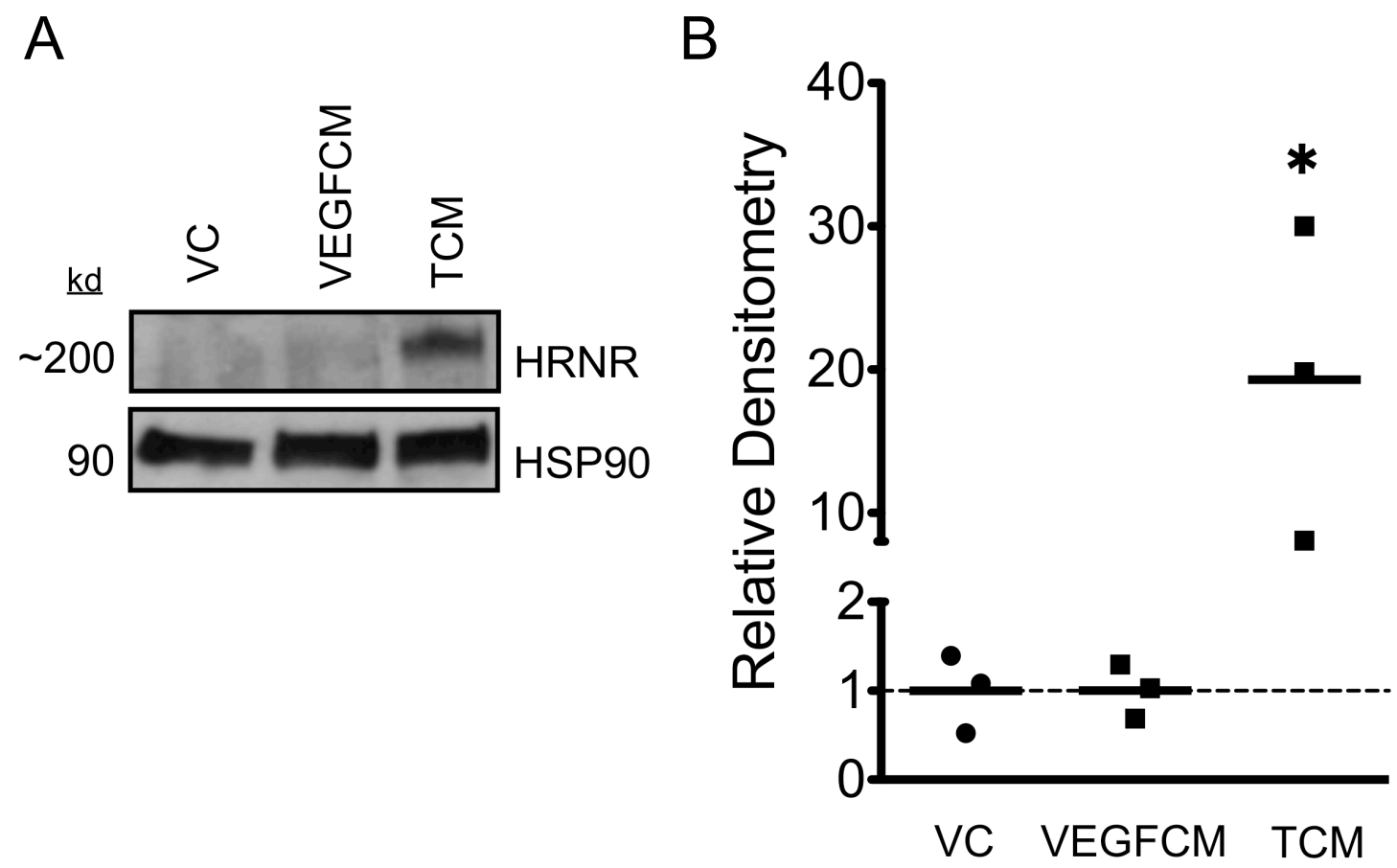

Figure 2.6 Hornerin is upregulated in TCM treated HUVEC whole cell lysates. A. Representative western blot of lysates from VC, VEGFCM, and TCM treated HUVECs. HUVECs were incubated with VEGF and TCM for 24 hours. HSP90 is shown as a loading control. Molecular weight in kilodaltons (kd) also shown. B. Relative densitometry of 3 separate experiments, measured as HRNR fold expression over HSP90. Data represented as mean. * $\mathrm{p}<0.05$ one-tailed, unpaired student's t-test compared to VC. 
2.3.5 Hornerin is upregulated in HUVECs in response to interleukin-12 (IL12) and basic fibroblast growth factor (FGF2)

As VEGF is not responsible for upregulating hornerin, we assessed the potential candidates for TCM induced hornerin expression using a human angiogenesis antibody array (Figure 2.7A). As expected, VEGF was the most abundant growth factor present in TCM. IL6, IL8, IL12, TNF, and FGF2 were also increased. In addition to VEGF, L3.6pl cells are known to express large amounts of FGF2 and IL8 (138), thereby confirming the ability of the human angiogenesis array to detect overexpressed growth factors. To assess the potential for the identified growth factors to upregulate hornerin, the top 5 most-expressed, non-VEGF growth factors were incubated with HUVECs at published B-max concentrations (IL6 (139), IL8 (140), FGF2 (141)). A representative blot and densitometric quantification of 2 separate experiments are shown in Figure 2.7B and $\mathrm{C}$, respectively. Hornerin expression was significantly increased in response to treatment with IL12 (1.8-fold over VC), FGF2 (1.5-fold over VC), but not IL6, IL8 or TNF (Figure 2.7B) or VEGF (Figure 2.6). While slightly elevated, IL6 and IL8 did not significantly increase hornerin expression, as measured by unpaired student's t-test. Further, pre-incubation with an FGFR inhibitor PD173074 decreased FGF-induced hornerin expression by 52\% (Figure 2.7D). Although FGF2 and IL12 induced hornerin expression, the relatively modest increases after incubation when compared with the robust upregulation after TCM incubation indicate the presence of other factors in the TCM important for hornerin regulation. Additionally, the coordination of several growth factors together might result in synergistic overexpression, similar to what was observed after incubation 
A

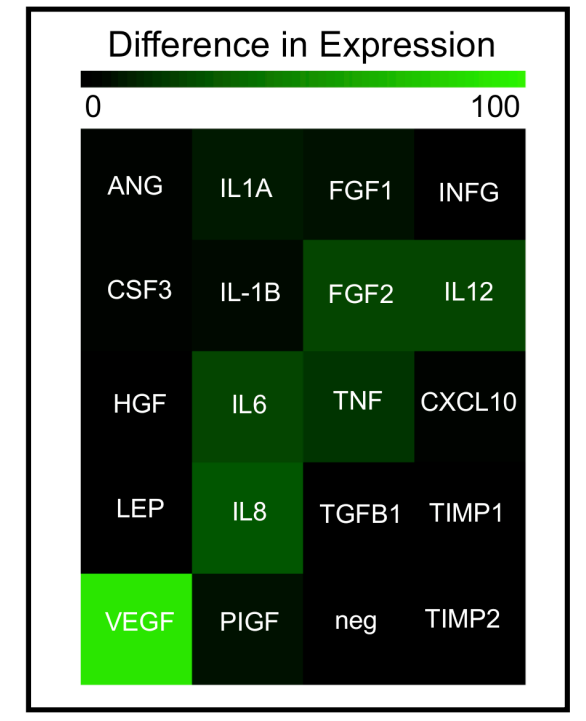

D

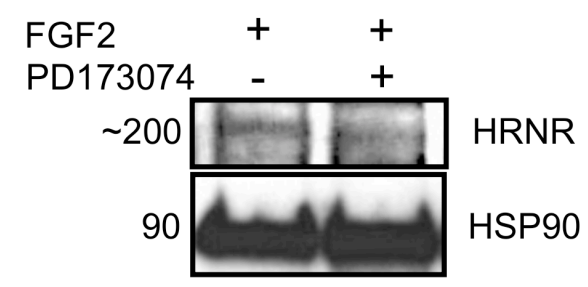

B

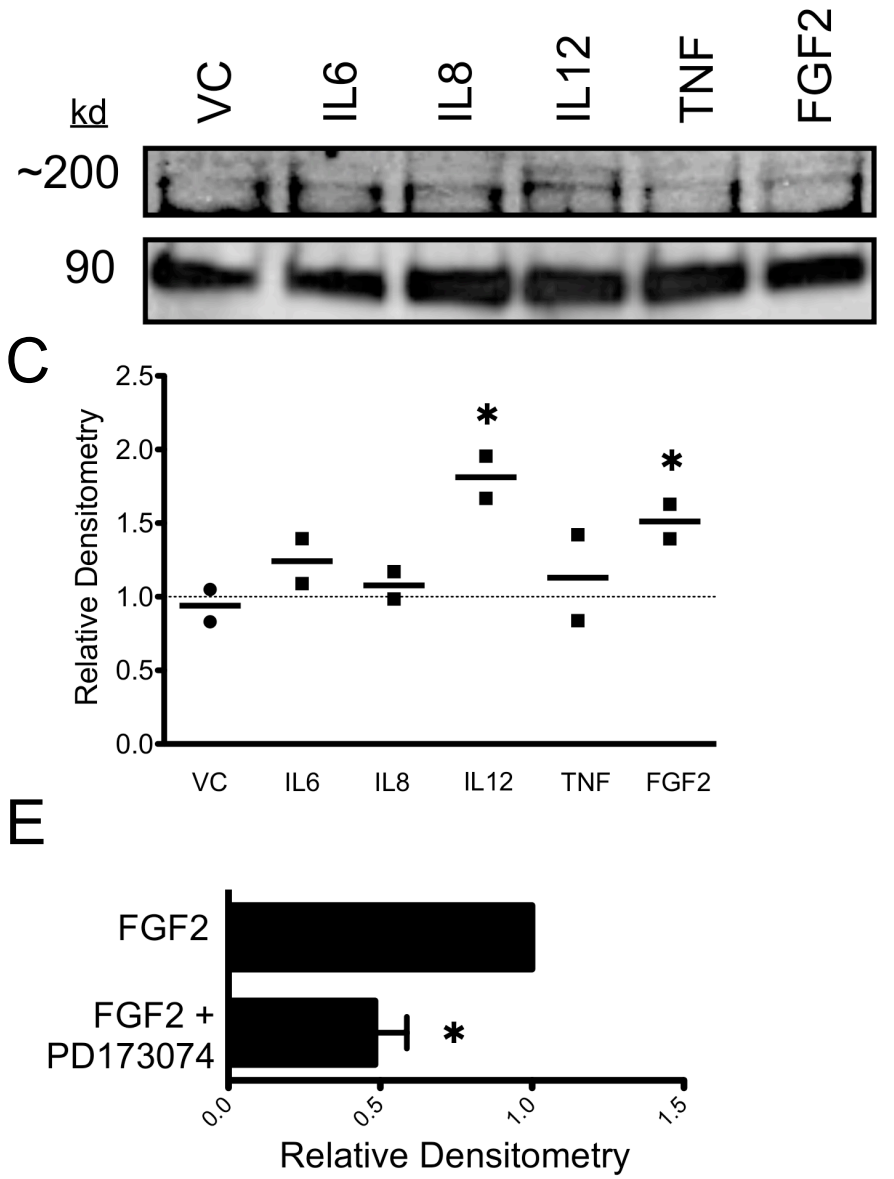

Figure 2.7 Hornerin is upregulated in HUVECs in response to interleukin-12 (IL12) and basic fibroblast growth factor (FGF2) A. Heatmap of the difference in expression of selected growth factors in TCM compared to VC media measured by Human Angiogenesis Antibody Array. "neg" refers to a negative control built into the assay by the manufacturer. B. Representative western blot of the lysates of HUVECs treated with the top 5 differentially expressed growth factors at B-max concentrations for 24 hours. HSP90 shown as a loading control. Molecular weight in kilodaltons (kd) also shown. C. Relative densitometry of 2 separate experiments measured as fold HRNR expression over HSP90. D. Western blot analysis of hornerin expression following 5 hour pre-treatment with PD173074 (an FGFR inhibitor) and subsequent stimulation by FGF2 for 24 hours. E. Relative densitometry of 3 experiments measured as fold HRNR over HSP90. * p < 0.05 compared to $\mathrm{VC}(\mathrm{C})$ or FGF2 (E) via one-tailed, unpaired students t-test. 
with TCM. Knowledge of expression modulators could direct further studies into the signaling and mechanism of hornerin in tumor vessel development, providing possible points of therapeutic intervention.

\subsubsection{Hornerin is specifically expressed in human PDAC vessels}

To determine whether hornerin upregulation was present in human tumors, we stained 10 resected PDAC tissues from de-identified patients for hornerin expression. Patient and tumor characteristics are listed in Table 2.1. The patients' genders were roughly split (60\% female, $40 \%$ male). Tumors were mostly moderately differentiated (Grade 2, 7 samples), with the remainder consisting of poorly differentiated tumors (Grade 3, 3 samples). No well-differentiated tumors were obtained in this study. After obtaining paraffin embedded tumors from the University of Virginia Biorepository and Tissue Research Facility (BTRF), 5 micron sections were prepared and hornerin staining and localization was assessed via immunohistochemistry. As shown in Figure 2.8A, normal pancreas endothelial (NPE) cells (arrows) lining vessels denoted with a "V", are negative for hornerin expression. Additionally, pancreatic acini are moderately stained. However, tumor endothelial (TE) cells (arrows) near PDAC (arrowheads) stain positively for hornerin (Figure 2.8B). Likewise, PDAC cells stain positive for hornerin. In fact, a myriad of solid tumors stain positive for hornerin, as assessed by a tumor microarray. The common subtypes of renal cell carcinoma (RCC) consistently had the most hornerin expression (Figure 2.9). Next a certified pathologist, Dr. Christopher Moskaluk quantified the extent of hornerin staining in each of the sections via histologic score, a metric 
Table 2.1 Characteristics of PDAC patients used in this study. De-identified patient data including age, gender, race and tumor grade were provided by the University of Virginia Biorepository and Tissue Research Facility.

\begin{tabular}{|c|c|c|}
\hline & & $\begin{array}{c}\text { Frequency } \\
\text { (Number) }\end{array}$ \\
\hline Age (years) & $\begin{array}{c}50-60 \\
60-70 \\
70-80 \\
>80\end{array}$ & $\begin{array}{l}50 \%(5) \\
30 \%(3) \\
10 \%(1) \\
10 \%(1)\end{array}$ \\
\hline Gender & $\begin{array}{c}\text { Male } \\
\text { Female }\end{array}$ & $\begin{array}{l}40 \%(4) \\
60 \%(6)\end{array}$ \\
\hline Race & White & $100 \%(10)$ \\
\hline $\begin{array}{l}\text { Tumor } \\
\text { Grade }\end{array}$ & $\begin{array}{c}1 \text { - Well Differentiated } \\
2 \text { - Moderately Differentiated } \\
3 \text { - Poorly Differentiated }\end{array}$ & $\begin{array}{l}10 \%(1) \\
20 \%(2) \\
70 \%(7)\end{array}$ \\
\hline
\end{tabular}



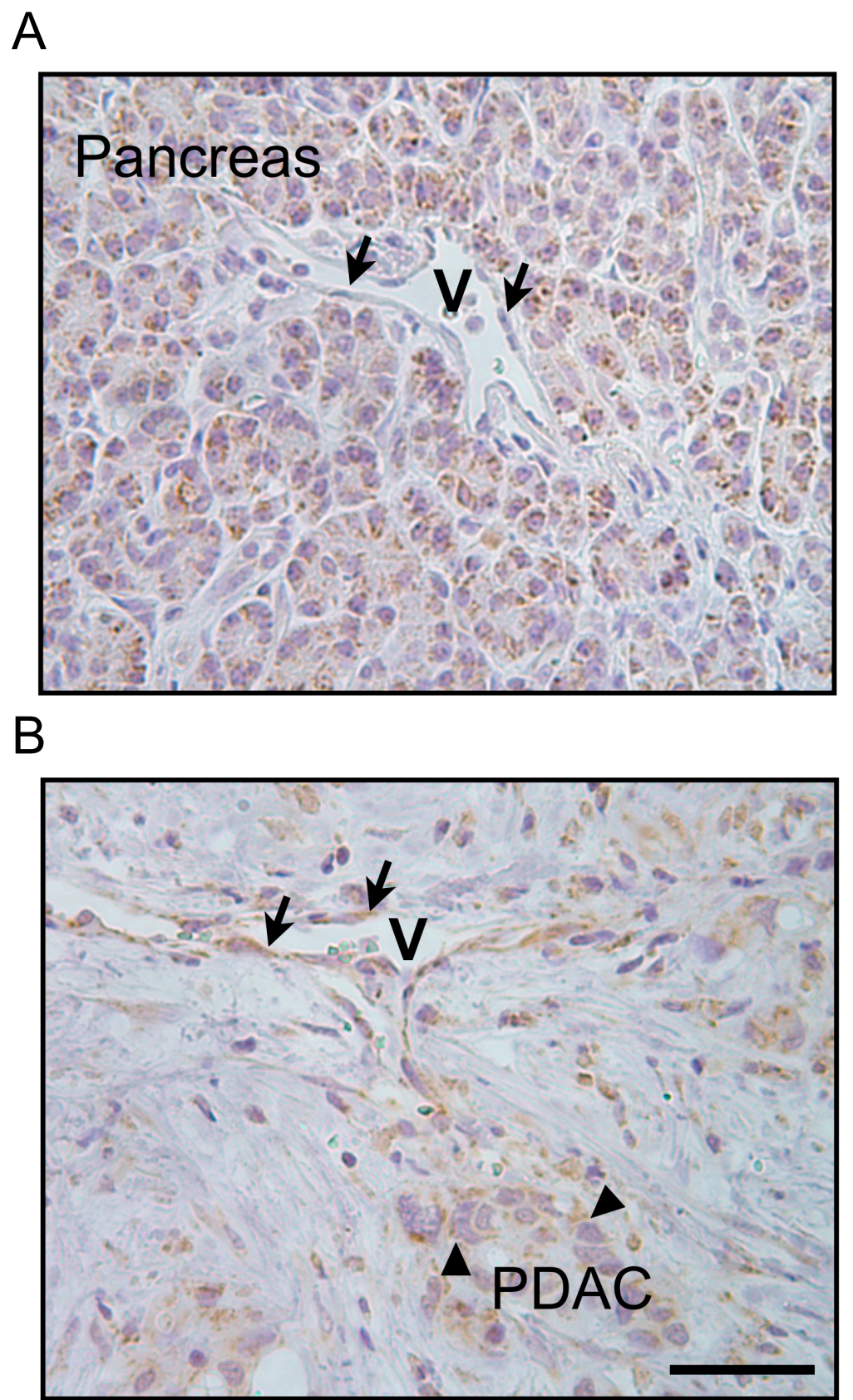

Figure 2.8 Hornerin is expressed in resected human PDAC associated tumors and vessels. A. Anti-hornerin DAB immunohistochemistry (IHC) on normal unaffected pancreas and vessels, "V" and B. PDAC (arrowheads) and associated vessels "V". A and B. Endothelial cells marked with black arrows. Scale bar: $100 \mu \mathrm{m}$. 


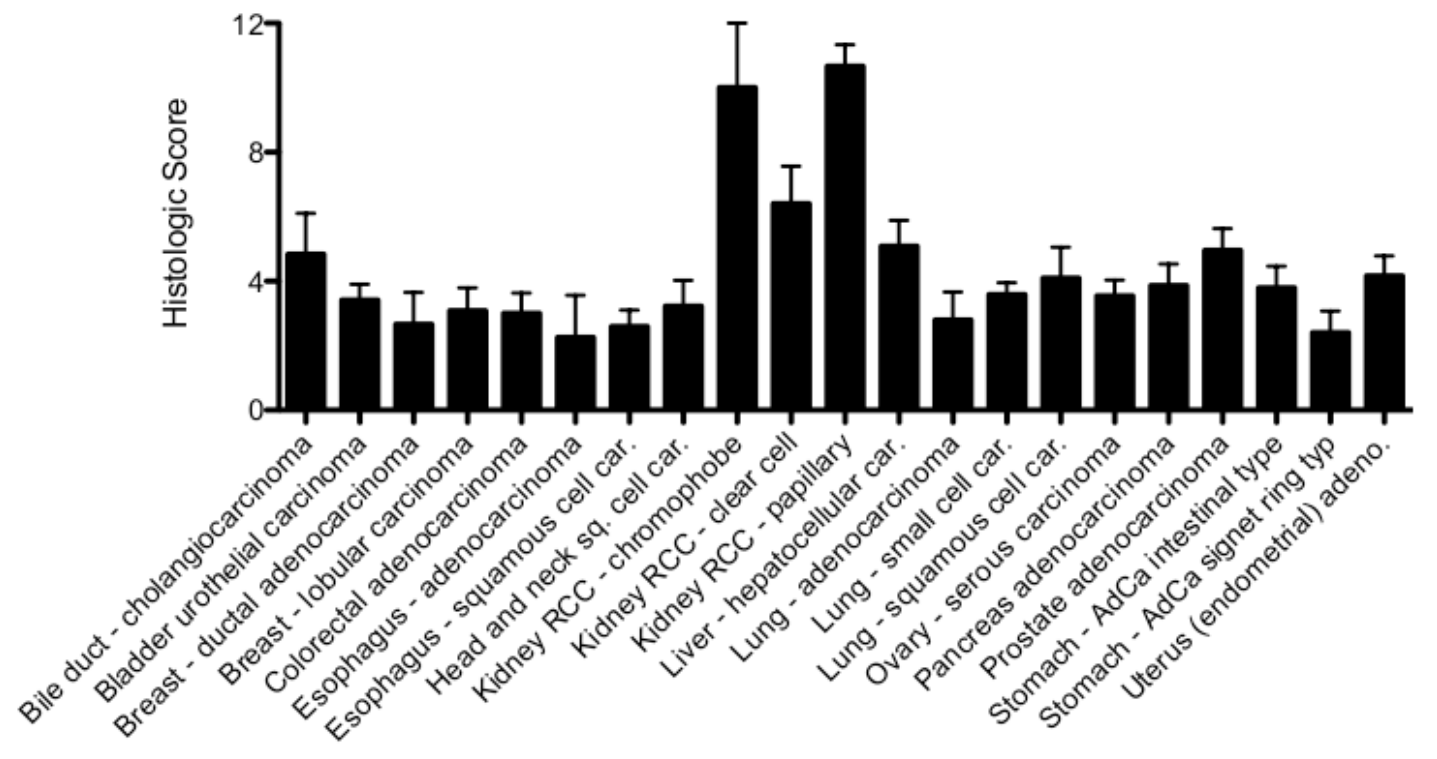

Figure 2.9 Hornerin is expressed in a variety of solid tumors. A tumor tissue microarray (TMA) was stained for hornerin expression and scored by a certified pathologist. The pathologist's histologic score was plotted for each tumor type. The number of scored samples for each type of tumor ranged from 2-12, but most tumor types had at least 6 samples scored. All data represented as mean \pm s.e.m. 

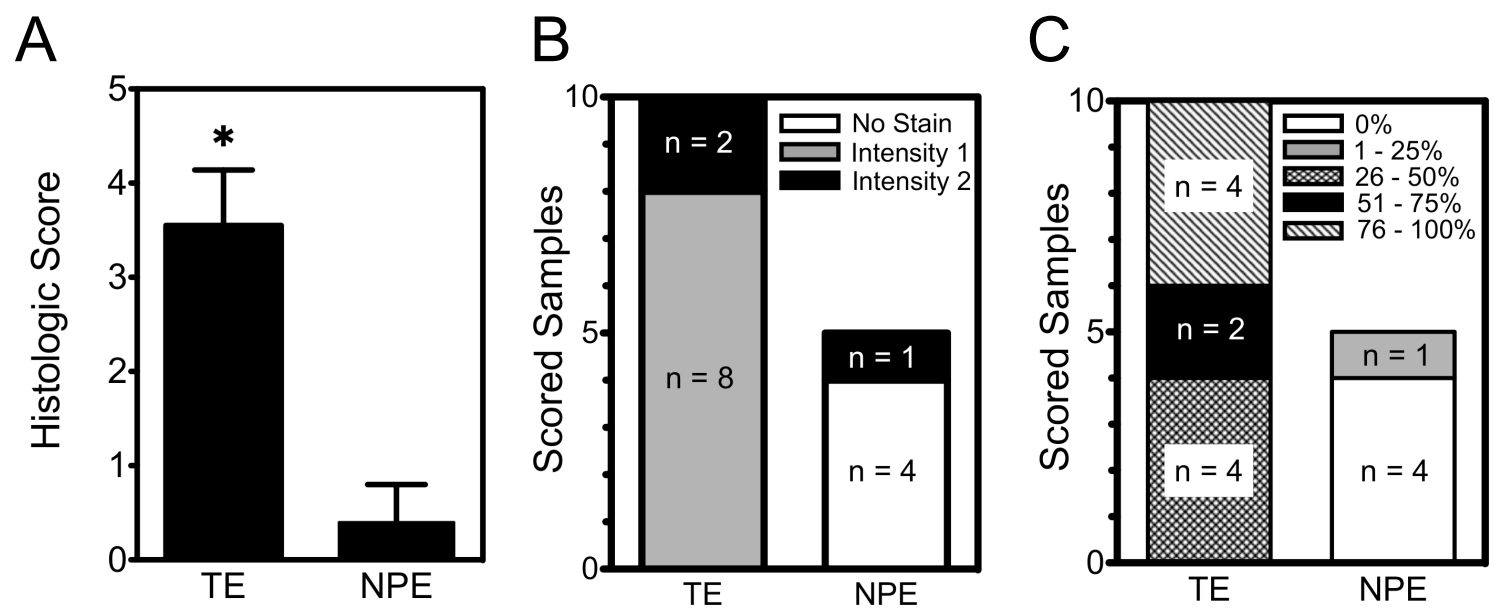

Figure 2.10 Quantification of PDAC and normal pancreas vessels. TE and NPE score A. Histologic score of tumor endothelium (TE) ( $n=10$ samples) and normal pancreas endothelium (NPE) ( $n=5$ samples) determined by certified pathologist. Histologic score is comprised of staining intensity and percent positive cells. B and C. Dissection of each histologic scoring metric - staining intensity and percent positive cells, respectively. Each $\mathrm{n}$ represents one patient sample. Data represented as mean \pm s.e.m. ${ }^{*} \mathrm{p}<0.05$ onetailed, unpaired student's t-test compared to NPE. 
that our laboratory has used in the past to quantify immunohistochemical staining (126). Vessel histologic score from the 10 PDAC cases is shown in Figure 2.10A; 5 of the 10 resected PDAC samples had significant areas of non-affected pancreas where NPE staining could be quantified. TE scored 7-fold higher than NPE, indicating hornerin expression specificity under pathologic conditions. Histologic score is comprised of staining intensity and percent positively stained cells in the sample. A breakdown of the samples in each scoring component revealed a large disparity between NPE and TE (Figures 2.10B and C). TE mostly stained with moderate intensity, while 2 samples stained with high intensity. All but one sample of NPE was negative for hornerin expression, the exception stained with moderate intensity (Figure 2.10B). In each PDAC sample, at least $25 \%$ of TE were positive for hornerin. In 4 PDAC samples, greater than $75 \%$ of tumor vessels stained positively. To the contrary, all but one NPE sample was negative for hornerin. The lone positive NPE sample had less than $25 \%$ of its vessels staining (Figure 2.10C). Although hornerin expression has specificity for tumor endothelium, staining of normal acini indicates that hornerin is not a good biomarker for PDAC; however, it was exciting that hornerin expression was positive in tumor endothelium but not normal pancreas endothelium, suggesting hornerin may play a role in PDAC vessels and potentially PDAC pathogenesis.

The identification of hornerin from our in vivo phage display-based functional proteomics screen follows the identification of plectin (116) and validates the use of these unbiased methods to identify previously uncharacterized proteins important in human disease. Verification of VEGF-pathway independence and confirmation of its presence in 
human PDAC tissue prompted us to investigate the function and mechanism of hornerin in tumor and tumor vessel pathogenesis.

\subsection{Materials and Methods}

\subsubsection{Statistics}

All p-values were calculated in excel using the one-tailed, unpaired student's t-test function. 2-way ANOVA statistical methods are described in full detail in a section below.

\subsubsection{Cell culture}

L36.pl cells were obtained as a gift from Dr. Craig Logsdon. L36.pl tumor cells were grown in minimal essential medium (MEM) with $10 \%$ fetal bovine serum (FBS) and penicillin/streptomycin and passaged every 72 hours. All experiments were completed with cells from passages 10 to 20. HUVECs were purchased from Lonza (Allendale, NJ) and grown in endothelial growth media (Lonza). HUVECs were split every third day and used from passages 2 to 9 . Both cell types were passaged and maintained in T-75 cell culture flasks until needed for experiments.

\subsubsection{Phage screen and sequencing}

$10 \mu \mathrm{L}$ of PhD-7 phage display library (New England Biolabs, Ipswich, MA) was suspended in $190 \mu \mathrm{L}$ of DPBS. The phage solution was injected via tailvein into mice harboring orthotopic pancreas L3.6pl tumors. To avoid extravasation, phage were al- 
lowed to circulate for 8-10 minutes before the mouse was sacrificed and cardiac perfused with $30 \mathrm{~mL}$ of DBPS. Tumors were dissected, minced into $1-2 \mathrm{~mm}$ pieces and phage were released by homogenization in a Dounce homogenizer in solution consisting of DPBS $+1 \%$ TritonX, EDTA and protease inhibitors (Thermo Scientific \#78410, Rockford, IL). Phage were amplified via manufacturer's (New England Biolabs) instructions, suspended in $200 \mu \mathrm{L}$ of DPBS and 2 additional rounds of screening were completed as described above.

After 3 rounds of screening, phage were titered (per New England Biolabs' instructions) and isolated from positive, blue plaques ( $\beta$-galactosidase) and placed in $50 \mu \mathrm{L}$ water to lyse host E. coli. $10 \mu \mathrm{L}$ of bacterial lysis/phage water solution was added as template for PCR amplification. PCR primers were designed to amplify the variable 21nucleotide peptide library coding sequence. Primer sequences were:

FWD 5' CCTTTAGTGGTACCTTTCTAT 3'

REV: 5' GCCCTCATAGTTAGCGTAACG 3'.

After PCR, the amplicon was sent to the UVA DNA Sciences core for sequencing using the same REV primer and 3730 DNA Analyzer (Applied Biosystems, Grand Island, NY).

\subsubsection{Phage-based ELISA}

HUVECs were plated in MaxiSorp 96-well plates (NUNC, Penfield, NY) - triplicate wells for each of 30 phage clones and 6 wells for controls. 24 hours after seeding, a 
change of media containing VEGF (at published B-max concentration (136)) or TCM was incubated for 24 hours before the initiation of the assay. Cells were washed, then $10^{10}$ isolated phage in $100 \mu \mathrm{L}$ DPBS were incubated in each well for 1 hour. After 3 DPBS $+0.1 \%$ Tween-20 (DPBST) washes, HRP-tagged anti-M13 antibody (1:3000; GE Healthcare, Pittsburgh, PA) was incubated for 30 minutes. The plates were washed 3 additional times (DBPST) then developed with $200 \mu \mathrm{L}$ 3,3',5,5'-Tetramethylbenzidine (TMB; Sigma T4444) for 5 minutes. Absorbance at $650 \mathrm{~nm}$ was measured on a microplate reader (Molecular Devices, Sunnyside, CA).

\subsubsection{Two-way ANOVA analysis}

Absorbance values were corrected by comparison to KE-phage (wild-type) controls. To validate and confirm which clones reliably bound TCM and/or VEGFCM over VC, 2-way ANOVA analysis on 2 separate experiments was used. The independent variables tested were conditioned media and the 2 sets of experimental data collected on different days. Additionally, an independent variable interaction term was calculated. To be classified as a TCM or both TCM and VEGFCM binder, clones must demonstrate significant difference resulting from conditioned media versus $\mathrm{VC}$, but not between experimental data sets or the interaction factor $(\mathrm{p}<0.01)$. 2-way ANOVA analysis was completed using MATLAB.

2.4.6 HUVEC lysates and immunocytochemistry 
To obtain lysates for western blots, HUVECs were seeded in $10 \mathrm{~cm}$ circular dishes and allowed to grow for 48 hours. Next, growth factors were added in a change of media at published B-max concentrations (IL6 (142), IL8 (143), IL12 (144), TNF (145), FGF2 (146) and cells were incubated for an additional 24 hours. Cells were then washed with DPBST 2 times, and lysed with RIPA buffer (\#89900; Thermo Scientific) in Halt Protease Inhibitor Cocktail (Thermo Scientific).

For immunocytochemistry experiments, cells were grown on 4 chamber glass slides (Thermo Scientific) and incubated with TCM or VEGFCM as described before. For immunostaining, cells were washed 3 times with DPBS, blocked with $2 \%$ bovine serum albumin (BSA) and then incubated with anti-hornerin antibody (1:100; \#HPA031469, Sigma-Aldrich, St. Louis, MO) at room temperature for 1 hour. After 3 washes with DPBST, FITC labeled anti-rabbit secondary (1:200, \#ab6717, Abcam, Cambridge, MA) was incubated for 30 minutes. After 3 washes with DPBST the chambers were removed and cells were mounted with PROLONG gold anti-fade reagent (Invitrogen, Grand Island, NY). Cells were imaged with a confocal microscope (Nikon TE 2000E2, equipped with a Melles Griot Argon Ion Laser System and Nikon D-Eclipse C1 accessories, Nikon Instruments, Melville, NY) using a $\times 60 / 1.45$ NA oil-immersion objective and EZ-C1 software (Nikon Instruments).

\subsubsection{Tumor conditioned media and implantation}


To prepare TCM, media was collected before every new passage (72 hours of production), passed through a cell culture $0.22 \mu \mathrm{m}$ syringe filter to remove dead cells and cell debris (Argos Technologies, Elgin, IL). After filtration, media was concentrated to 10X using Ultra-10 $10 \mathrm{kd}$ molecular weight cutoff centrifugation filter units (Millipore, Billerica, MA) and stored at $-20^{\circ} \mathrm{C}$ until needed.

For L36.pl tumor implantation, cells were allowed to grow under normal culture conditions for 72 hours before being lifted with trypsin, counted and suspended in aliquots of 500,000 cells in 50 $\mathrm{L}$ L Hanks Balanced Salt Solution (HBSS). $50 \mu \mathrm{L}$ of Matrigel (BD Biosciences, Franklin Lakes, NJ) was added to each cell suspension aliquot in a 1:1 ratio just before implantation. Cell-Matrigel slurry was injected subcutaneously on the flanks of athymic nude mice or orthotopically into the pancreas and the injection site was monitored daily for growth. The orthotopic injection protocol was adapted from previously published methods (147) and described in the supplemental methods of a vessel quantification paper published by our group (148).

\subsubsection{FITC-labeling of phage clones}

Phage were amplified according to instructions in the PhD-7 peptide phage display kit from New England Biolabs and brought up in concentrations of $1 \times 10^{9}$ plaque forming units (pfu) $/ \mu \mathrm{L}$ in DPBS. For each labeling experiment, $2 \mu \mathrm{L}$ of $12.5 \mu \mathrm{g} / \mu \mathrm{L}$ of FITC solubilized in DMSO was added to $200 \mu \mathrm{L}$ of phage DPBS solution was incubated in the dark with gentle rocking for 1 hour at room temperature. After incubation, the labeled phage were purified by 3 rounds of PEG precipitation and resuspended in $200 \mu \mathrm{L}$ of 
DPBS. $200 \mu \mathrm{L}$ of FITC-labeled phage were injected via tailvein and allowed to circulate for 4 hours before the animal was cardiac perfused and the tumored pancreas was removed, cryopreserved and sectioned. This protocol was adapted from previously described methods (117).

\subsubsection{Western Blot and Angiogenesis Antibody Array}

For western blotting, HUVEC lysate concentrations were measured via BCA assay (Pierce, Rockford, IL) and adjusted so that equal concentrations were loaded into each well. Samples were loaded into precast 4-15\% TGX polyacrylamide gels (Bio-Rad, Hercules, CA) and separated by electrophoresis at 200V in tris/glycine/SDS (TGS) running buffer. Proteins were transferred to nitrocellulose membranes via iBlot transfer system (Invitrogen) following manufacturer's instructions. Membranes were then washed in Tris-buffered saline $+1 \%$ Tween-20 (TBST), blocked for 30 minutes in $2 \%$ milk TBST solution. Primary antibody (anti-hornerin, \#ab78909, Abcam; anti-HSP-90, \#4877, Cell Signaling Technology, Danvers, MA) was diluted 1:200 in TBST and incubated with gentle rocking at $4^{\circ} \mathrm{C}$ overnight. After overnight incubation, membranes were washed for 10 minutes 3 times, then HRP-labeled secondary (anti-rabbit, \#NA934V, GE Healthcare, Waukesha, WI) was diluted 1:5000 and incubated for 45 minutes with gentle rocking at room temperature. The membrane was then washed 3 times for 10 minutes each. Protein-antibody conjugation was detected by Immbolion chemiluminescent detection kit (Millipore, Billerica, MA) and exposed on HyBlot autoradiography film (Denville Scientific, Metuchen, NJ). Exposed films were scanned using a tabletop office scanner and 
converted to JPEG files for densitometry. Relative densitometry was computed using the "Gel Tool" in the NIH image processing software suite, ImageJ.

Major constituents of TCM were determined via Human Angiogenesis Antibody Array (Affymetrix, Santa Clara, CA) kit per manufacturer's instructions. TCM and VC media were incubated on separate test strips, then assayed for elevated growth factors in TCM relative to VC. After development on HyBlot autoradiography film, the film was scanned then TCM and VC densitometry were determined by ImageJ and absolute difference between TCM and VC was computed.

2.4.10 De-identified human PDAC sample collection, immunohistochemistry, and pathologic scoring

De-identified resected human PDAC specimens were obtained through the UVA Biorepository and Tissue Research Facility (BTRF). 10 formalin-fixed, paraffin embedded tumor samples were cut into $5 \mu \mathrm{m}$ sections. Sections were deparaffinized via washes of xylene and ethanol, then treated with low $\mathrm{pH}$ antigen unmasking solution (Vector Labs, Burlingame, CA) in a microwave for 20 minutes.

Tumor sections were then washed twice with DPBS. Next endogenous peroxidase activity was quenched using $0.5 \% \mathrm{H}_{2} \mathrm{O}_{2}$ in methanol for 30 minutes at room temperature. Next, slides were washed with DPBS then blocked for 30 minutes with DPBS $+5 \%$ (blocking buffer). Slides were then incubated with anti-hornerin antibody (\#HPA031469, Sigma) in blocking buffer (1:100 dilution) at $4^{\circ} \mathrm{C}$ overnight. After 3 
washes with DPBS, slides were incubated with anti-rabbit HRP-labeled secondary antibody (GE Healthcare, \#NA934V) diluted 1:2500 in blocking buffer for 30 minutes at RT. Signal was developed using DAB tablets (Thermo Fisher Scientific, Waltham, MA) per manufacturer's instructions. Next, slides were washed with DPBS and counterstained with hemotoxylin (Thermo Fisher Scientific) for 15 seconds, before being washed for 5 minutes under running tap water. Sections were then dehydrated in $3100 \%$ ethanol washes, followed by $2100 \%$ xylene washes, before mounting with CytoSeal 60 (\#23244257, Thermo Scientific).

Stained slides were scored by a certified pathologist according to the intensity of stain and percentage of positive cells. Endothelial cells were identified by a combination of cell morphology, nucleus shape and presence of erythrocytes. Histological images were taken on an Olympus BX-41 microscope, equipped with a U-RFL-T high-powered Olympus mercury burner and a QImaging Retiga-2000R camera. 
CHAPTER 3: HORNERIN EXPRESSION IS IMPORTANT IN TUMOR PROGRESSION AND VESSEL DEVELOPMENT 


\section{$\underline{3.1 \text { Abstract }}$}

Before therapies directed against hornerin can be developed, its functional role in tumor progression and tumor vessel development must be known. Therefore, we treated PDAC tumors with both monotherapy consisting of siRNA mediated hornerin knockdown and combination therapy combining hornerin knockdown with VEGF inhibition. Hornerin siRNA knockdown monotherapy resulted in a decrease in tumor volume compared to scrambled control siRNA injected tumors. We suspected the differences were the result of changes in tumor vasculature since hornerin was identified and validated as an important tumor endothelial protein. Consequently, parameters such as vessel volume fraction (VVF), fractal dimension, vessel radius and pericyte coverage were examined. When this study began, semi-automatic vessel analysis freeware programs did not exist, so we created a MATLAB based vessel analysis program called Rapid Analysis of Vessel Elements (RAVE) to aid our analysis of tumor vessels. Using RAVE, we observed a decrease in VVF, fractal dimension and vessel radius in the hornerin siRNA treated tumors compared to scrambled controls. Additionally, there was an increase in vessel coverage of alpha-smooth muscle actin (ACTA2) positive pericytes, indicative of a more mature vessels. Dynamic contrast-enhanced (DCE) magnetic resonance imaging (MRI) was used to study the functional differences between hornerin siRNA treated and untreated vessels. We observed marked decreases in tumor vessel function after siRNA treatment Next, we combined hornerin therapy with anti-VEGF therapy to investigate whether targeting compensatory or redundant pathways could produce enhanced tumor and tumor vessel abatement. Indeed, combining therapies resulted in an additive tumor volume de- 
crease and further reduction of key vessel parameters. These findings confirm the role of hornerin in tumor progression and provide rationale for the development of therapies against hornerin to be administered alone or in combination with currently approved antiVEGF therapies to improve overall patient survival.

\subsection{Introduction}

Understanding the functional consequences of protein expression in vascular development is essential to the design and development of successful anti-vascular therapies. Traditionally, metrics such as VVF, fractal dimension and vessel radius have been used to characterize and track the successes or failures of anti-vascular therapies (149). In the university or small research lab setting, most in vivo or ex vivo vessel analysis is completed manually with minimal computer assistance, resulting in laborious and time consuming analyses. Vessel analysis software is commercially available with some software packages being sold as optional add-ons to certain microscope systems such as Nikon's NIS-Elements (www.nis-elements.com) or Mauna Kei Technologies' Cellvizio (www.maunakeatech.com). However, these programs are costly and not open sourced to all, making them difficult to manipulate and adapt based on specific research needs. Some researchers have published manipulations to commercially available software (150, 151), but their efforts are not accessible unless the respective software has been purchased. Because MATLAB is a common engineering and science computing language accessible to most investigators, an open-source vessel analysis tool designed in MATLAB can be used and/or customized easily by all who are interested. Therefore we de- 
signed a MATLAB software package capable of semi-automatically analyzing vessel images called Rapid Analysis of Vessel Elements (RAVE). RAVE quickly and accurately analyzed vessel images from hornerin and scrambled control siRNA treated tumors, showing that VVF, fractal dimension, and vessel radii were reduced in hornerin versus control siRNA treated tumors.

Subsequent to ex vivo vessel structural vessel analysis, we completed in vivo MRI functional imaging of hornerin and control siRNA treated tumors to assess whether changes were actually reducing the function of tumor vessels. Since tumors need vessels to provide their oxygen and nutrients $(11,12)$, disturbing this supply line is paramount to ensuring successful anti-cancer therapies. We showed decreased tumor uptake of MRI contrast agent gadolinium (Gd-DTPA) and volume transfer coefficient $\left(\mathrm{K}_{\text {trans }}\right)$ in the hornerin treated tumors when compared to controls.

The use of combination therapies consisting of VEGF and some other compensatory or redundant pathways has shown success in preclinical tumor studies. Hanahan's group has focused on the FGF pathway to augment traditional anti-VEGF therapies (81, 82). We treated tumors with a combination of anti-hornerin and anti-VEGF therapies to test the hypothesis that redundant pathways were responsible for lackluster anti-VEGF clinical results. Interestingly, combination treatment regimen reduced tumor volume, VVF, fractal dimension and vessel radii as measured by RAVE more than either monotherapy alone, establishing the need to develop anti-hornerin therapies to augment existing standard of care anti-VEGF therapies. 


\section{$\underline{3.3 \text { Results and Discussion }}$}

\subsubsection{Detecting angiogenic changes in vessel architecture using RAVE}

The RAVE graphical user interface used in these experiments is shown in Figure 3.1. Briefly, the file to be analyzed is selected by clicking on the "Browse" button. Once the image is loaded, it will appear on all three image panes (Original Image, Binary Image, and Skeletonized Image). These image panes can be used to visually inspect how well the thresholded binary and skeletonized images represent the Original Image. The Threshold, Gaussian Threshold (G Threshold), H Size, Sigma and Maximum Radius parameters can be adjusted. This GUI drastically cuts down on measurement time. RAVE analysis takes as little as 20 seconds per image compared to 20 minutes to 1 hour with the conventional manual measurement approaches. Using this software, we were able to analyze a large set of images and dissect the changes in vessel architecture resulting from tumor vessel treatment with hornerin siRNA.

3.3.2 Knockdown of hornerin leads to decreased tumor burden and key vessel parameters

As hornerin is present in tumor vessels but not normal vessels, we hypothesized that hornerin may play an important role in tumor vessels and tumor progression. Therefore, we decreased endothelial hornerin expression via intratumoral injections of mousespecific siRNA in a subcutaneous L36.pl tumor model (cells of human origin). The choice of tumor model ensured that observed effects were a result of endothelial cell manipulation. Remarkably, intratumoral injections of hornerin siRNA resulted in tumors that 


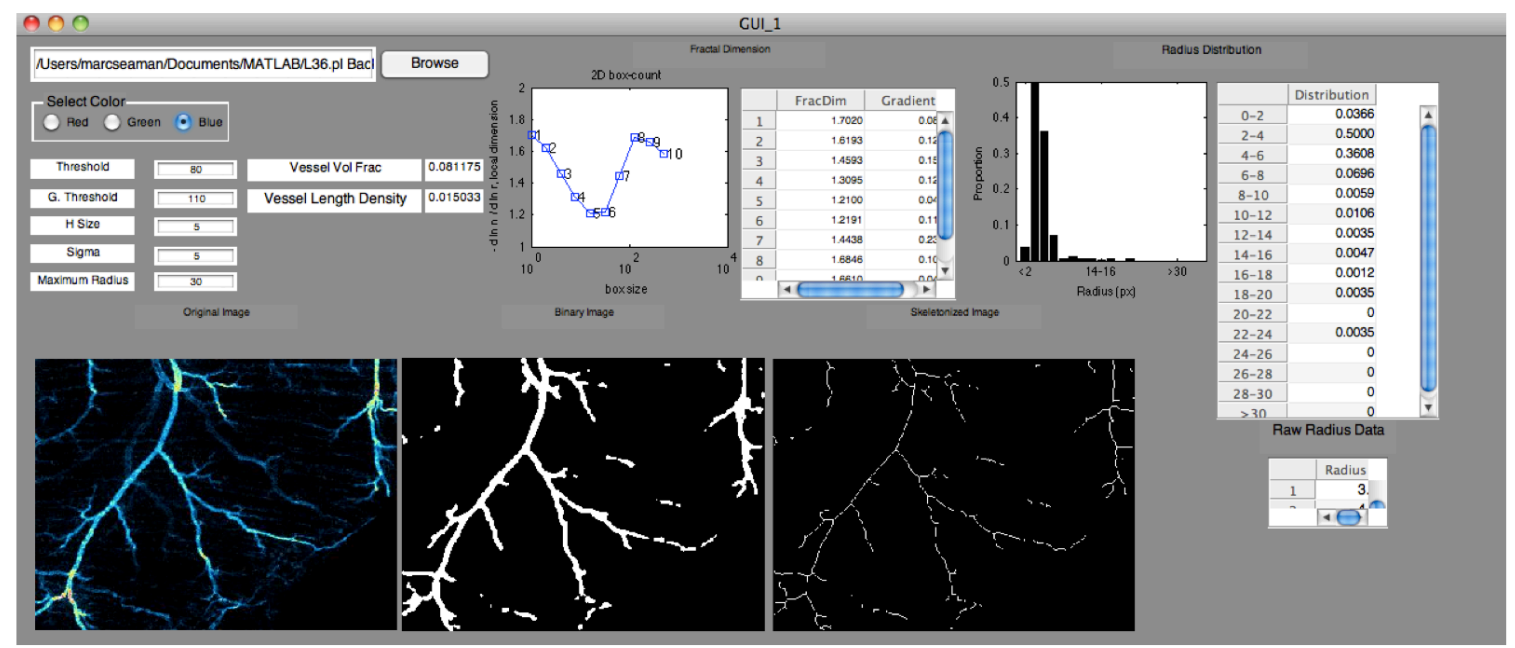

Figure 3.1 Overview of RAVE GUI. A representative screenshot of RAVE . The user is able to input parameters in the editable fields on the left and monitor the binarization and skeltonization of the image before ending the fitting process and recording data. The "Binary Image" is shown after being binarized, smoothed using a Gaussian filter, then binarized again. 

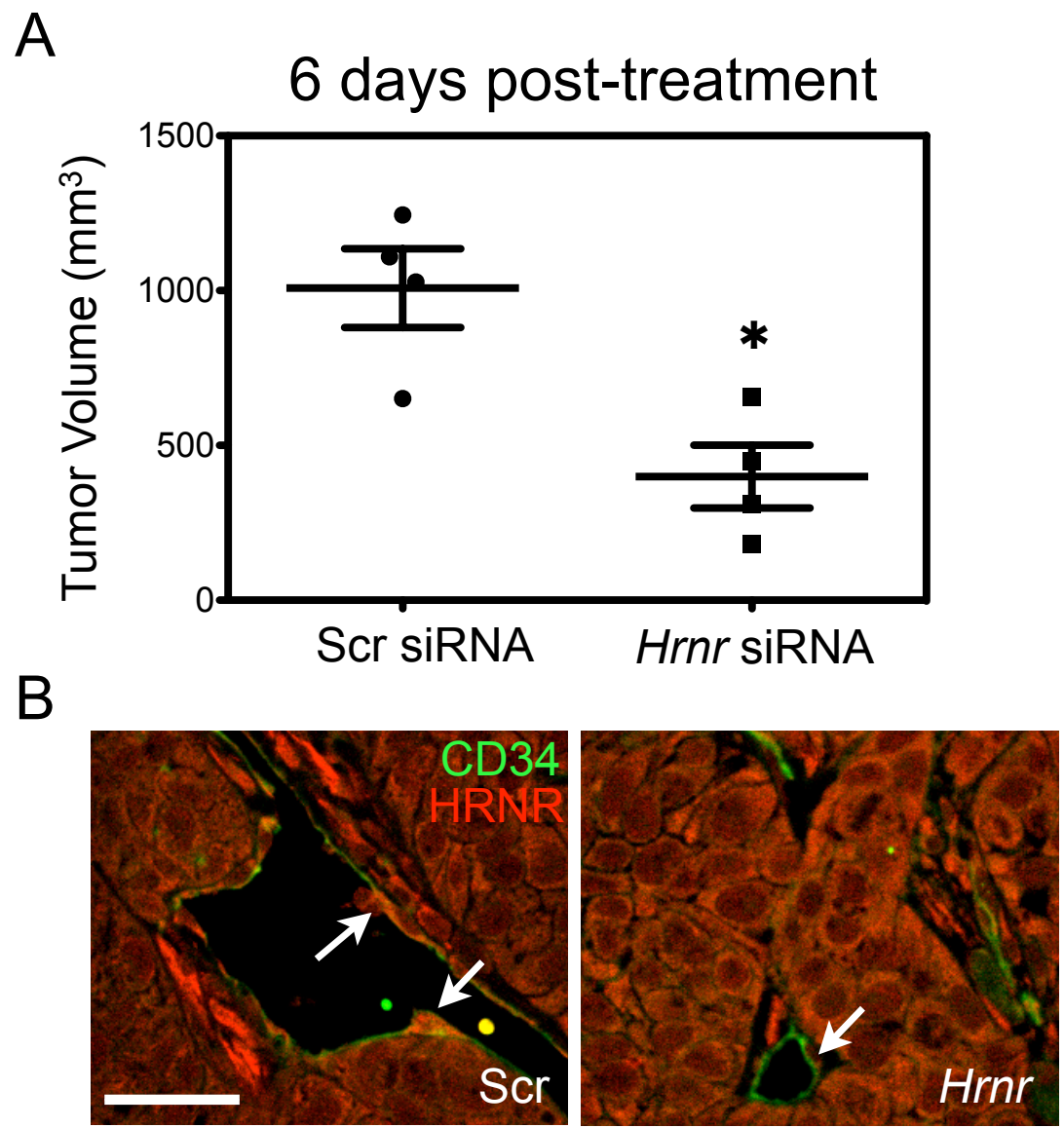

Figure 3.2 Hornerin knockdown results in decreased tumor volume. A. Tumor volume was measured upon termination of the experiment (Day 13 post-implantation). Tumors were injected with either hornerin (Hrnr) or scrambled (Scr) siRNA every other day starting on Day 7 post implantation. $\mathrm{n}=4$ tumors in each group. B. Representative image of vessels from Scr and Hrnr siRNA injected tumors confirm decreased HRNR expression in Hrnr CD34-positive tumor vessels and increased expression in Scr tumors vessels. Endothelial cells of note are highlighted with arrows. Scale bar: $50 \mu \mathrm{m}$. 


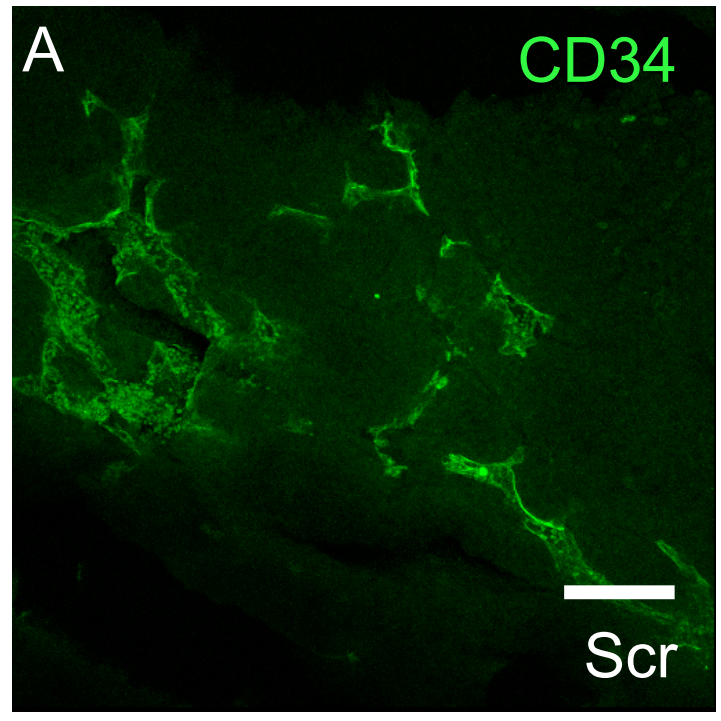

Figure 3.3 Hornerin knockdown results in decreased VVF and fractal dimension, but no change in the number of vessels per field. A. Representative thick section Z-stacked images of tumor vessels from Scr and Hrnr siRNA treated tumors. Z-stack thickness: $50 \mu \mathrm{m}$. B. The number of CD34positive vessels per $0.4 \mathrm{~mm}^{2}$ field was quantified in $5 \mu \mathrm{m}$ sections. $\mathrm{n}=16$ each group (4 tumors in each treatment group, 4 images per tumor). C. and D. 14 Hrnr and 14 Scr tumor thick section Z-stacks were analyzed by RAVE (4 tumors in each treatment group, 3-4 images per tumor). Vessel parameters such as C. vessel volume fraction, D. fractal dimension (a measure of tortuosity) are shown. ** $\mathrm{p}<0.05$ - one-tailed, unpaired student's t-test compared to Scr siRNA. n.s. - not significant. All data are represented by mean \pm s.e.m. Scale bar: A. $100 \mu \mathrm{m}$.
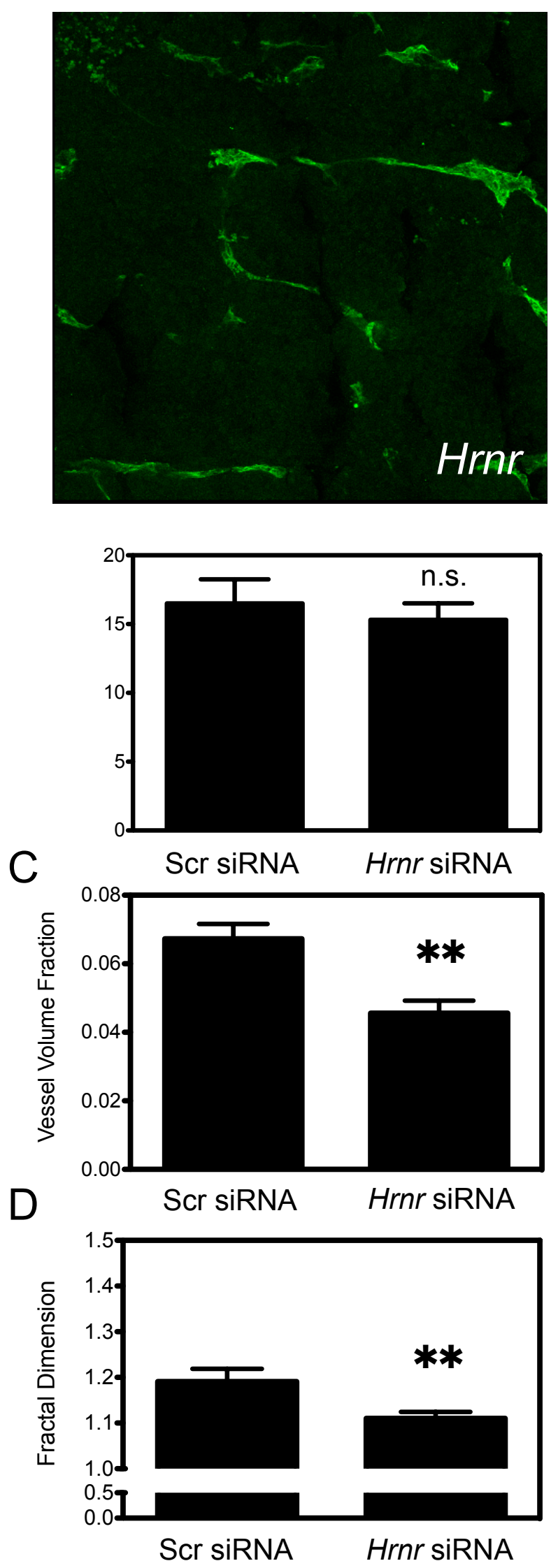
were 2.5 -fold smaller compared to tumors that were injected with control scrambled siRNA (Figure 3.2A). To ensure that hornerin was indeed knocked down, we looked for hornerin vessel (CD34-positive) expression in each of the treated tumors. Tumor vessels treated with Scr siRNA maintain high expression of hornerin, while hornerin expression in Hrnr siRNA treated vessels was greatly reduced (Figure 3.2B). CD34 immunofluorescent analysis on the tumors revealed that vessels in hornerin siRNA injected tumors had drastic differences in appearance i.e.- smaller radius and length, less sprouting, and reduced tortuosity (Figure 3.3A). However, the number of vessels per field was not statistically different (Figure 3.3B). We used RAVE (148) to measure vessel volume fraction (VVF), vessel radius and fractal dimension (a measure of tortuosity), parameters important in tumor vessels and vessel normalization (149). Hornerin siRNA injection reduced VVF and fractal dimension (Figure 3.3C and D). The VVF parameter is computed by dividing the total number of vessel pixels in by the total number of pixels in a given image field. Therefore, VVF is sensitive to changes in vessel number or vessel radius. A reduction in VVF without a decrease in the number of vessels, suggests that vessel radius was decreased. We found an overall shift in vessel radius from larger to smaller vessels (Figure 3.4), more evidence that tumor vessel progression has been halted. Evidence suggests that pericyte-endothelial interactions may play a role in vessel morphology and functionality in tumors (149), so we investigated ACTA2 expression in tumor associated pericytes.

A less mature, sprouting vessel is not highly invested by alpha-smooth muscle actin (ACTA2) positive pericytes $(149,152)$. In fact, colorectal patients with high 


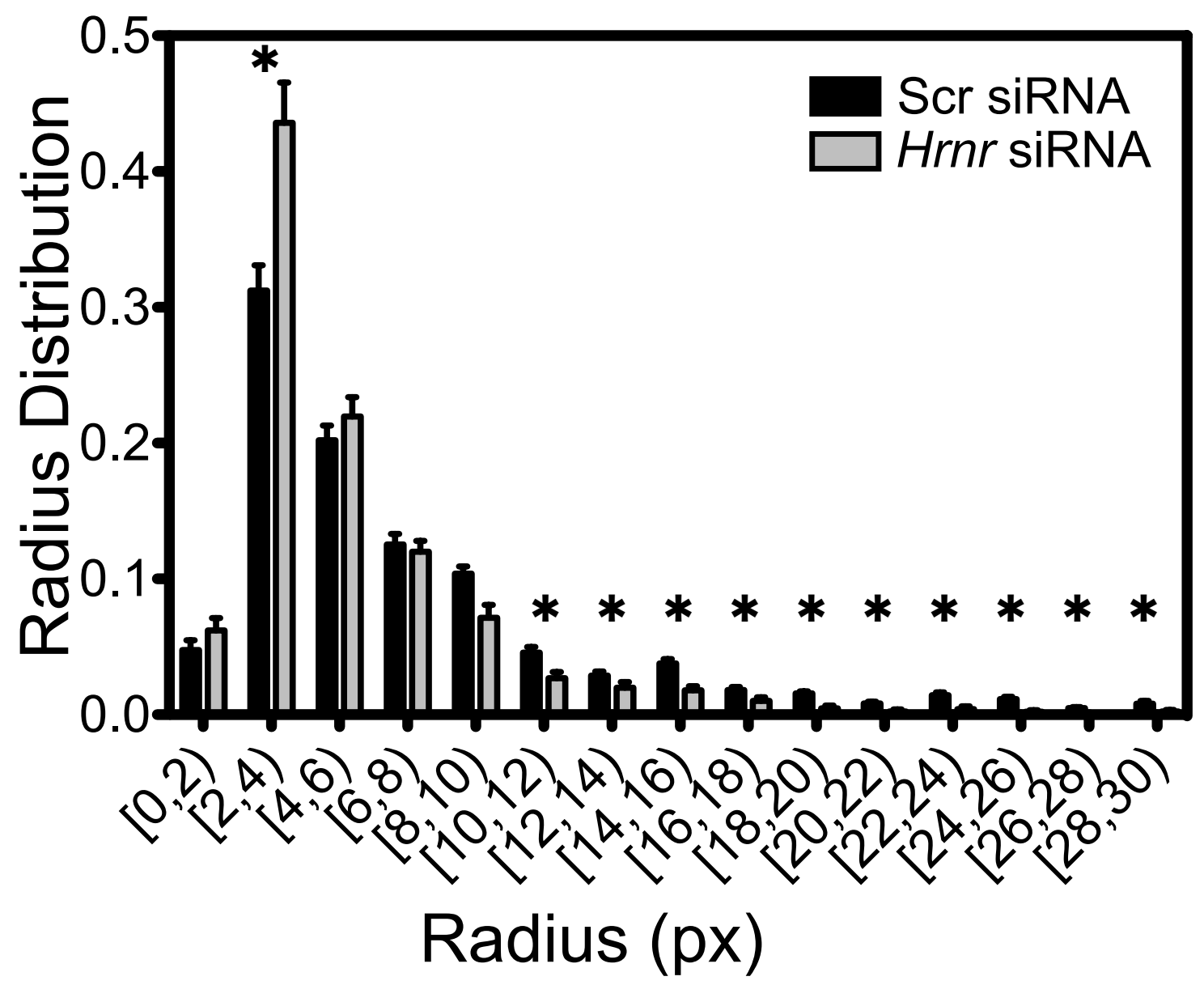

Figure 3.4 Hornerin knockdown results in a shift to smaller vessel radii. Radius distribution is shown. Vessels in tumors treated with Hrnr siRNA are over represented in the smaller pixel bins compared to Scr treated controls. All data are represented by mean \pm s.e.m. $* \mathrm{p}<0.05$ compared to Scr siRNA. 
A
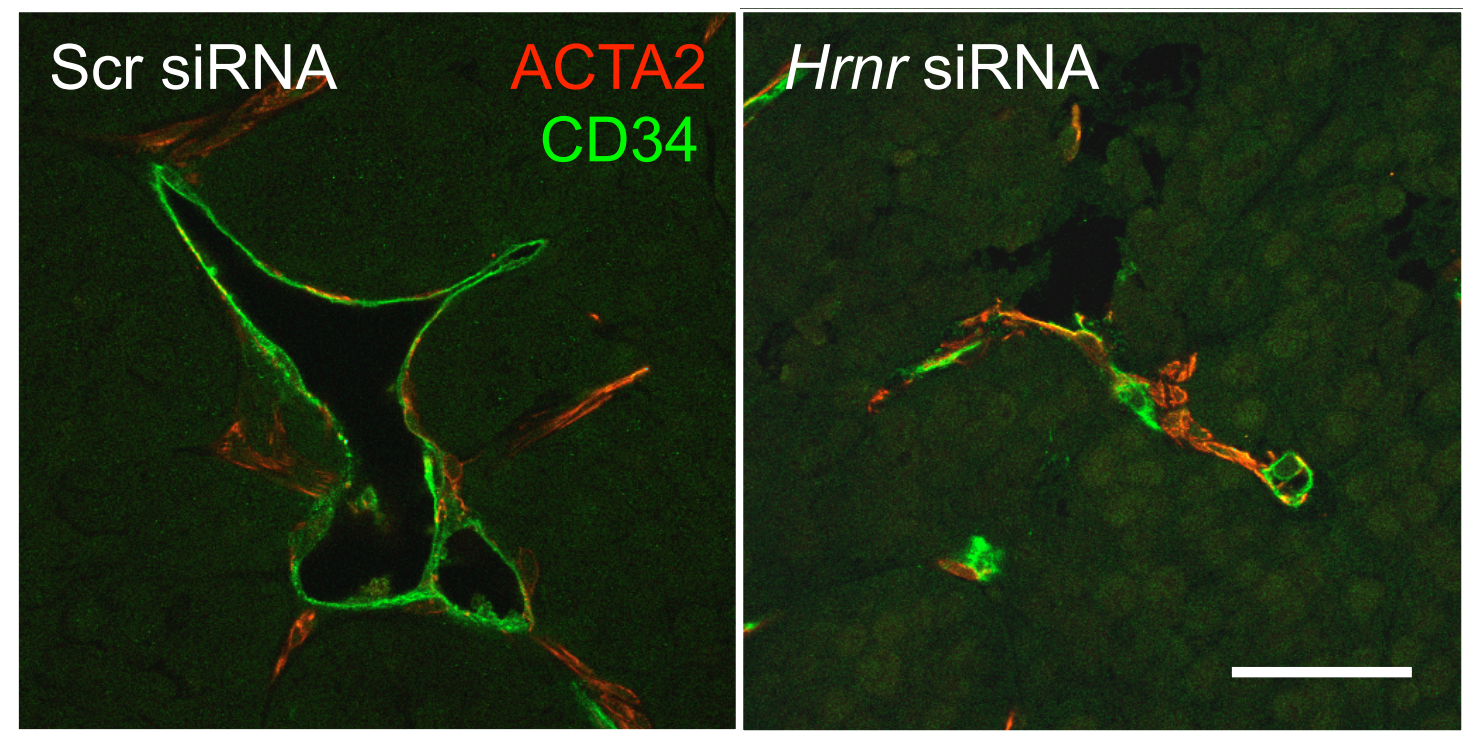

B

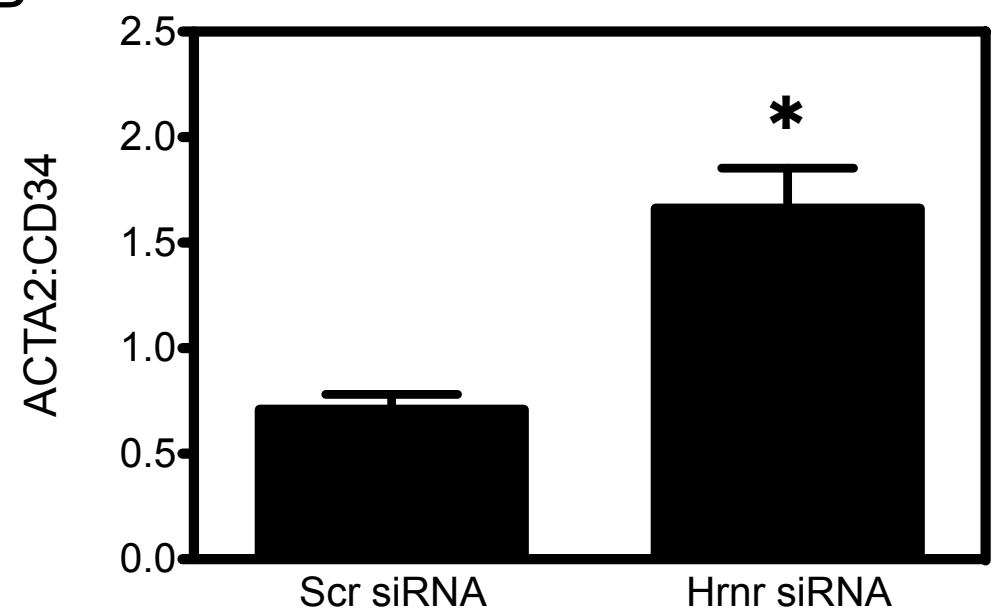

Figure 3.5 Hornerin knockdown increases ACTA2-positive pericyte coverage. A. Representative image of ACTA2 coverage on CD34-positive tumor vessels. B. Ratiometic quantification of 18 sections from Hrnr siRNA treated tumors (4 tumors, 3-5 sections per tumor) and $20 \mathrm{Scr}$ siRNA sections (4 tumors, 5 sections per tumor) were measured by dividing the number of ACTA2 positive pixels by CD34-positive pixels. All data are represented by mean \pm s.e.m. ${ }^{*} \mathrm{p}<0.05$ compared to Scr siRNA. Scale bar: $50 \mu \mathrm{m}$. 
microvessel density (MVD) and poor microvessel pericyte coverage index (MPI) had a significantly lower survival rate, compared to patients with high MVD and high MPI (152). We observed that hornerin siRNA injected tumor vessels had 2.3-fold increased ACTA2 coverage (Figure 3.5A and B), a result similar to previous vessel normalization studies (153). The cumulative data suggests that the number of vessels is not decreasing, however, important vessel parameters such as VVF, tortousity, pericyte recruitment, and radius are affected by hornerin knockdown.

3.3.3 Functional imaging reveals reduced vascularity and perfusion in hornerin siRNA treated tumors.

Tumor vessels serve as a supply line to a growing tumor. Consequently, disruption of this supply line should reduce tumor volume - the major hypothesis behind antiangiogenesis therapies. Ex vivo vessel parameters such as vessel number, VVF and vessel radius provide excellent insight into the structural and anatomic changes that occur as a result of therapeutic intervention; however, in vivo functional studies of tumor vascularity and perfusion are needed to determine if the delivery of oxygen and nutrients is actually reduced. Functional tumor vessel imaging through dynamic contrast-enhanced (DCE) magnetic resonance imaging (MRI) is an emergent, non-invasive tool to track tumor vessel development (154-156). Therefore, we treated tumors with Scr or Hrnr siRNA and observed functional changes through DCE-MRI. Once appropriately sized tumors developed, mice were imaged before treatment to establish baseline levels of vascularity. As expected, no difference was observed in the pre-treament images (Figure 3.6, 

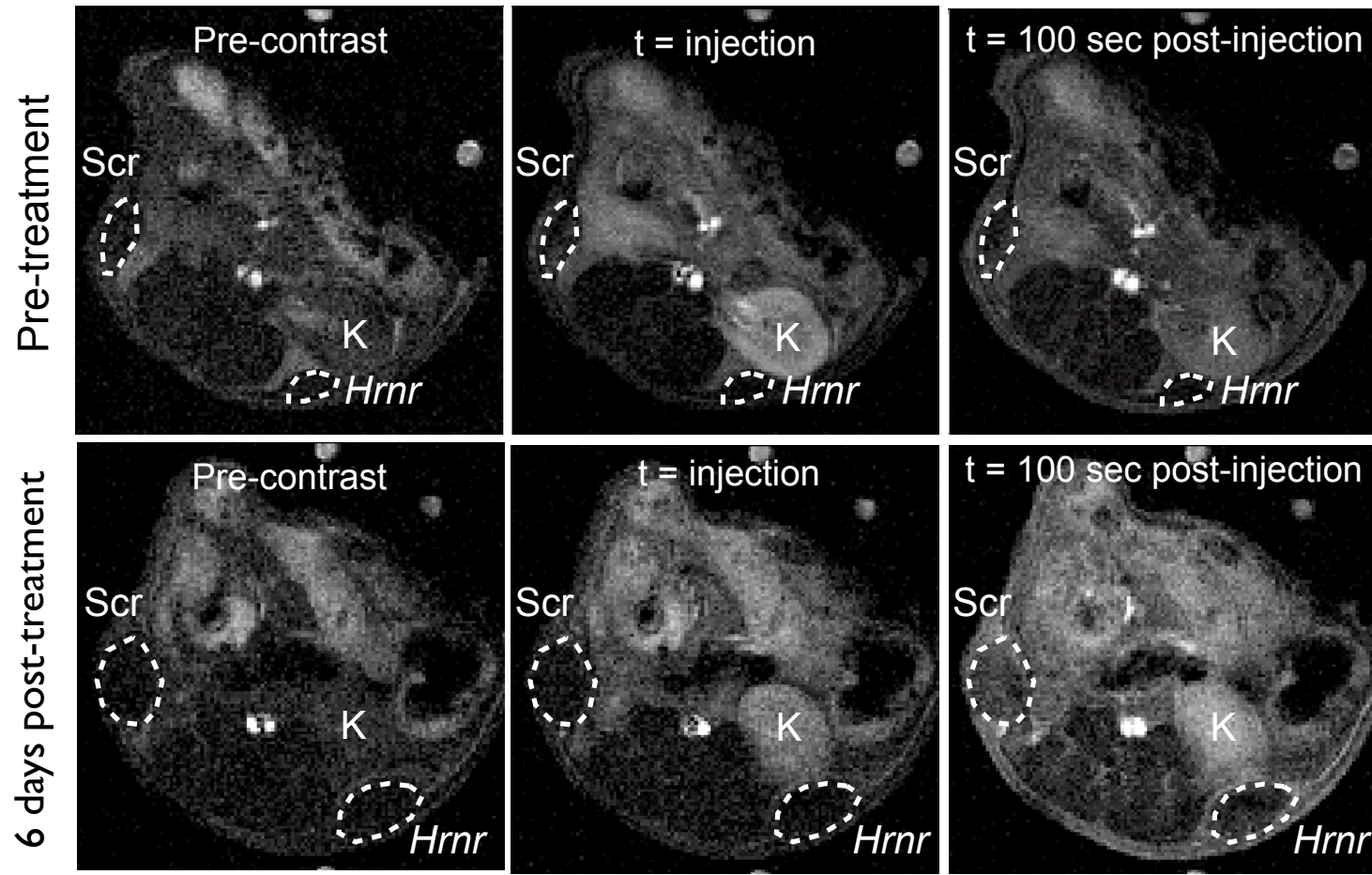

Figure 3.6 Functional studies by dynamic contrast-enhanced magnetic resonance imaging (DCE-MRI) reveal decreased vascularity in Hrnr siRNA treated tumors. Representative axial time course images of Gd-DTPA uptake taken pre-siRNA treatment (top) and 6 days post-MRI treatment (bottom). "Scr" represents scrambled siRNA injected tumors and and "Hrnr" represents Hrnr siRNA injected tumors. Dotted line demarcates tumor region of interest (ROI). The kidney " $\mathrm{K}$ " is labeled for reference. 

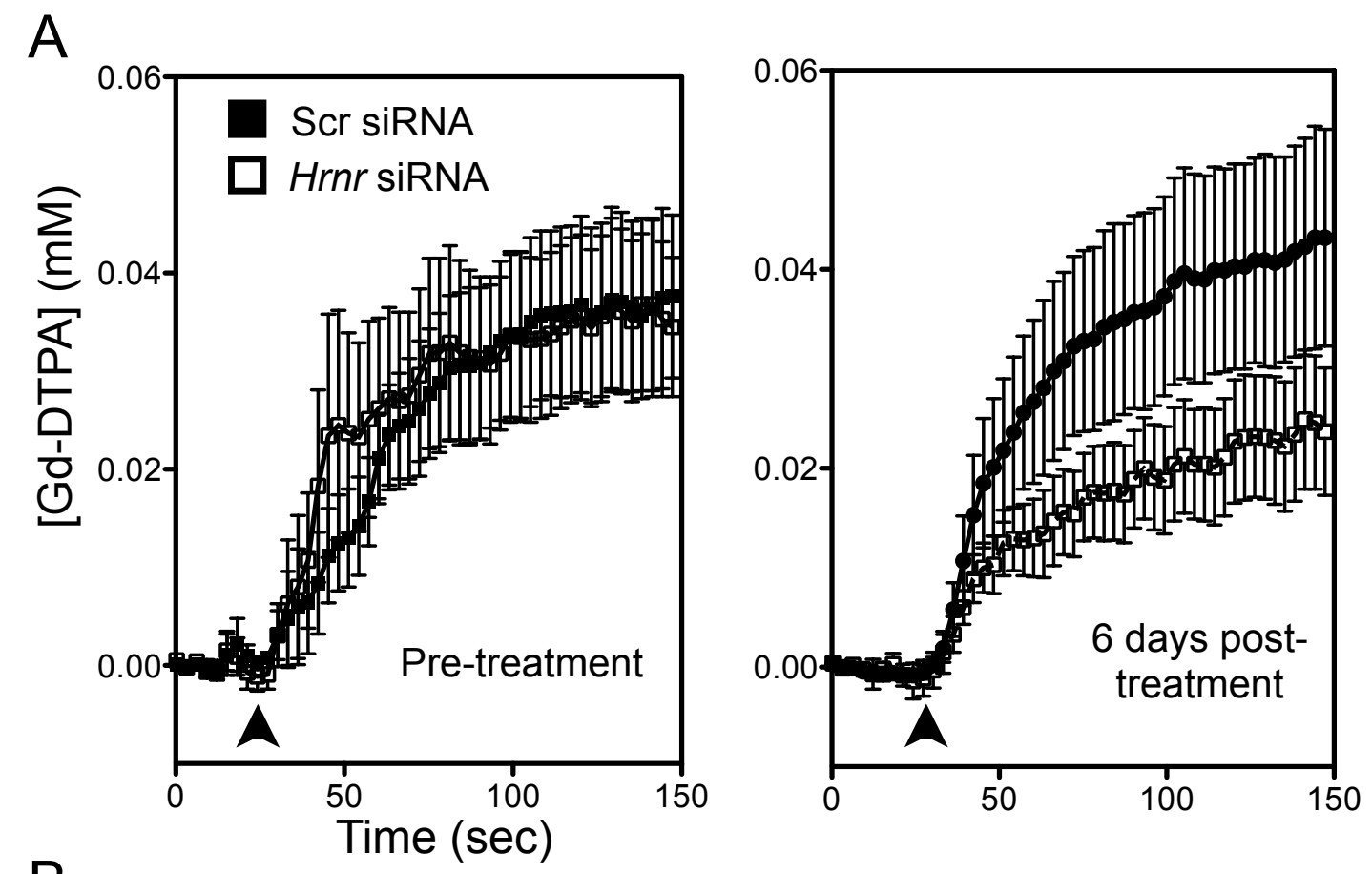

B

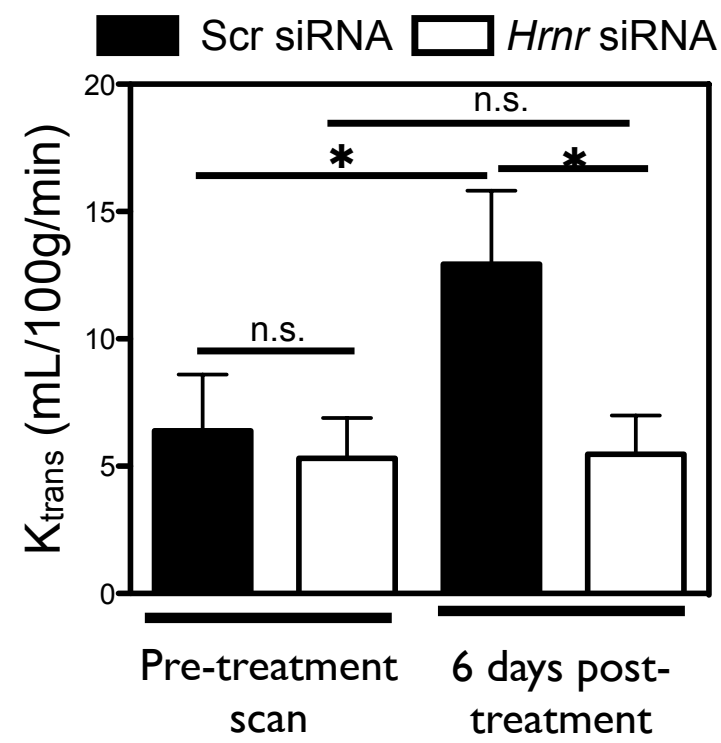

Figure 3.7 Gadolinium-DTPA (Gd-DTPA) uptake and volume transfer coefficient $\mathbf{K}_{\text {trans }}$ are reduced in $\mathbf{H r n r}$ siRNA treated tumors. A. The concentration of Gd-DTPA $(\mathrm{mM})$ in the tumors was plotted over time $(\mathrm{n}=7$, each treatment group). Scans were completed on mice before siRNA treatment (left) and 6 days post-treatment (right). Time of Gd-DTPA injection is signified by arrowhead. B. Volume transfer coefficient $\mathrm{K}_{\text {trans }}$ was calculated for both pre-treatment and post-treatment tumors $(n=7$, each treatment group). All data are represented by mean \pm s.e.m. $* \mathrm{p}<0.05$. n.s. - not significant. 
top), gadolinium-diethylenetriamine penta-acetic acid (contrast agent, Gd-DTPA) time

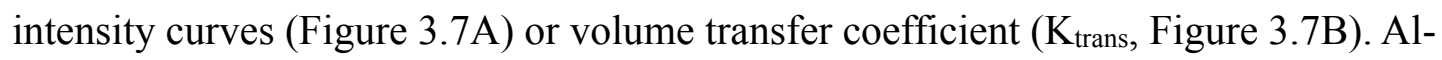
though tumors did not receive treatment prior to pre-treatment scans, they are labeled as Scr or Hrnr to indicate their subsequent treatment designation in Figures 3.6 and 3.7A and B. Next, tumors were randomly selected to receive Hrnr or scrambled siRNA. Importantly, after just 6 days of treatment, large differences were observed in Hrnr and Scr siRNA injected tumors. After 100 seconds, Gd-DTPA signal intensity was noticeably increased in the Scr siRNA treated tumors compared to the Hrnr siRNA treated tumors (Figure 3.6). The Gd-DTPA time intensity curves separate 15-20 seconds after injection and tumor concentration of Gd-DTPA remains 2-fold reduced in Hrnr siRNA injected tumors through the entire time course (Figure 3.7A). Likewise, after 6 days of siRNA injections, a 2.4-fold reduction in $\mathrm{K}_{\text {trans }}$ is observed in Hrnr tumors compared to Scr control (Figure 3.7C). These data confirm that knockdown via Hrnr siRNA treatment results in reduction of both structural and functional parameters, affecting the ability of a tumor to maintain unchecked growth.

3.3.4 VEGF inhibitor AV-951 combined with hornerin knockdown produced an additive reduction in tumor growth and vessel development.

Because hornerin expression is not regulated by VEGF, we investigated whether combining hornerin knockdown and VEGF inhibition would produce an enhanced therapeutic response. For these experiments, we used a VEGFR2 inhibitor currently in Phase II clinical trials, AV-951 $(156,157)$. The treatment schedule is used in our study is 
A

siRNA Injected $\quad$ AV-951 or control administered by oral gavage

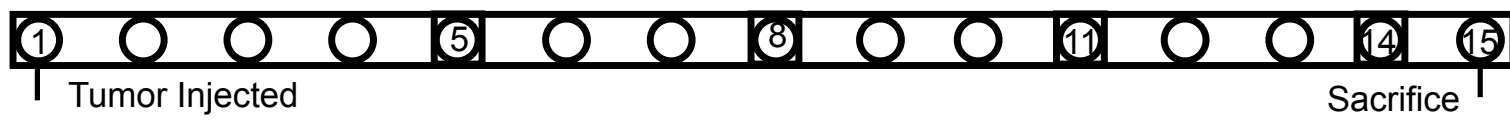

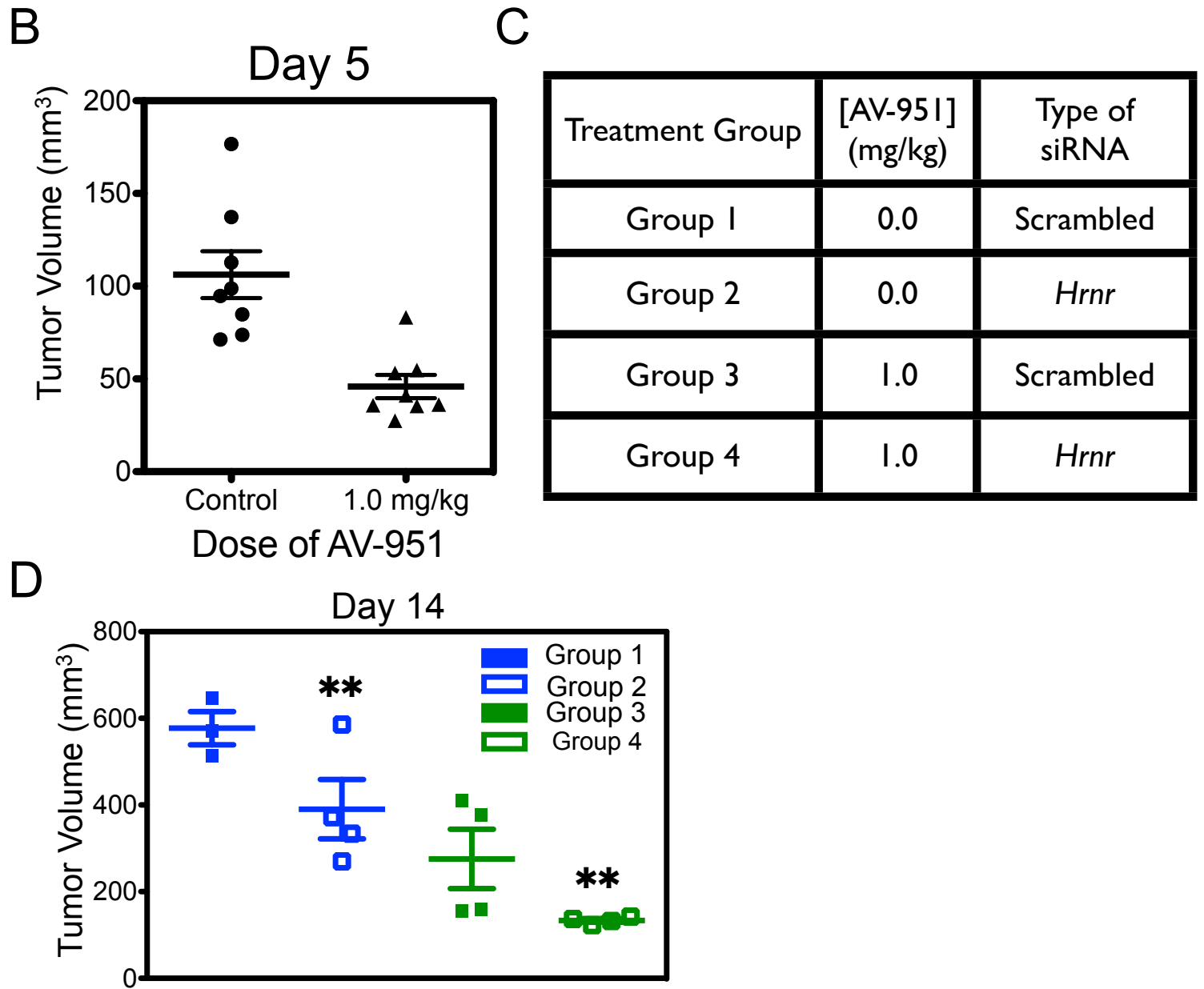

Figure 3.8 Hornerin and VEGF combination treatment results in increased tumor volume and tumor vessel abatement. A. Timeline showing points of treatment over the course of the 15-day experiment. B. Tumor volume ( $n=8$ in each treatment group) was measured on Day 5 post-injection (before siRNA treatment). C. Treatment regimen for each group, Groups 1-4. On Day 5, control and $1.0 \mathrm{mg} / \mathrm{kg}$ treated tumors were randomly selected to receive either scrambled (Scr) or Hrnr siRNA, resulting in 4 treatment groups. D. Tumor volume was again measured at Day 14, post-tumor implantation. $n=4$ in each treatment group, except Group $1(\mathrm{n}=3)$. See Methods for explanation of outlier exclusion. All data are represented by mean \pm s.e.m. ${ }^{* *} \mathrm{p}<0.005$. 

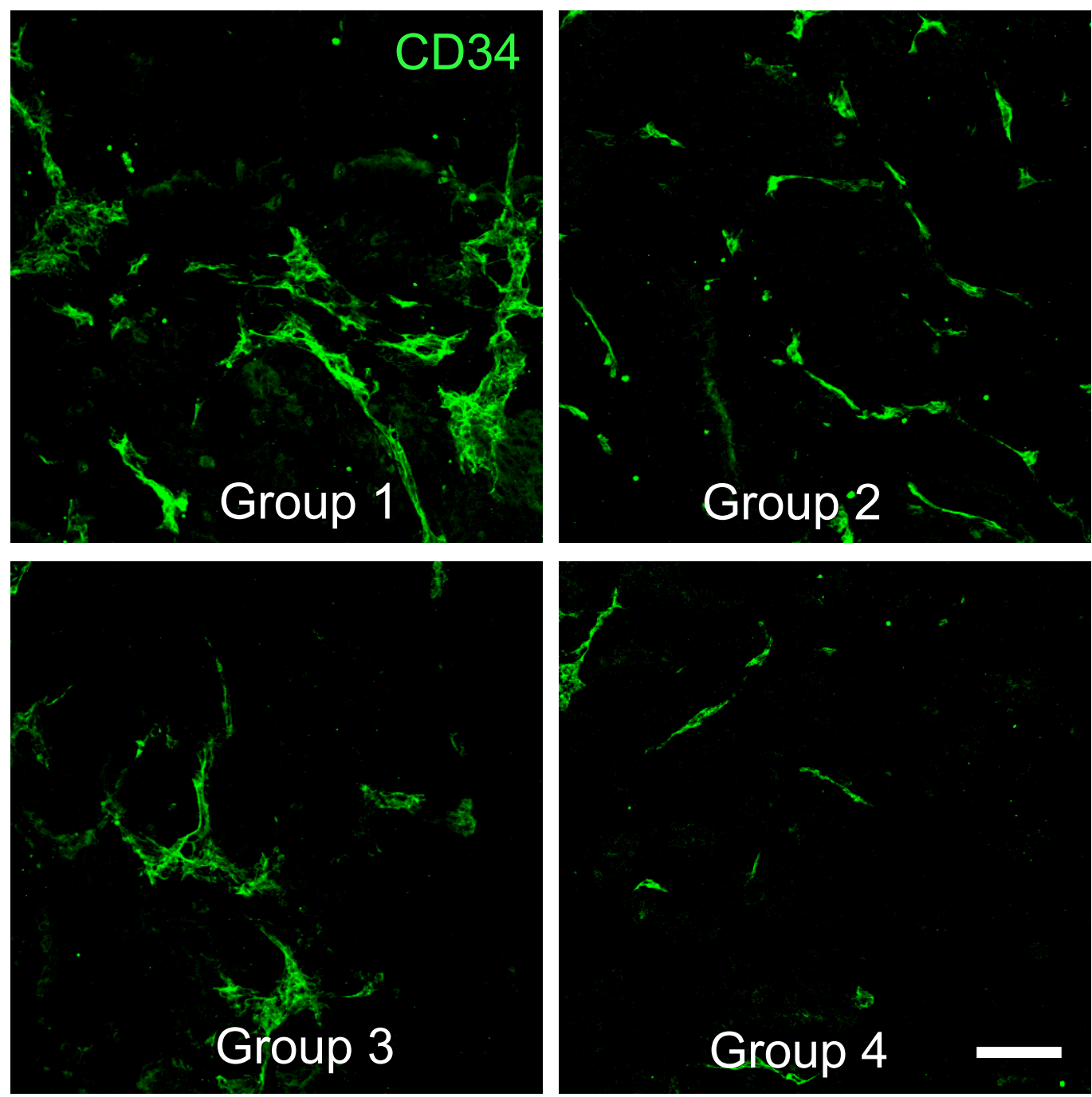

Figure 3.9 AV-951 and hornerin combination therapy produces vessels with varying morphology. Representative thick section Z-stacked images of CD34-positive vessels from each treatment group. Scale Bar: $100 \mu \mathrm{m}$. 


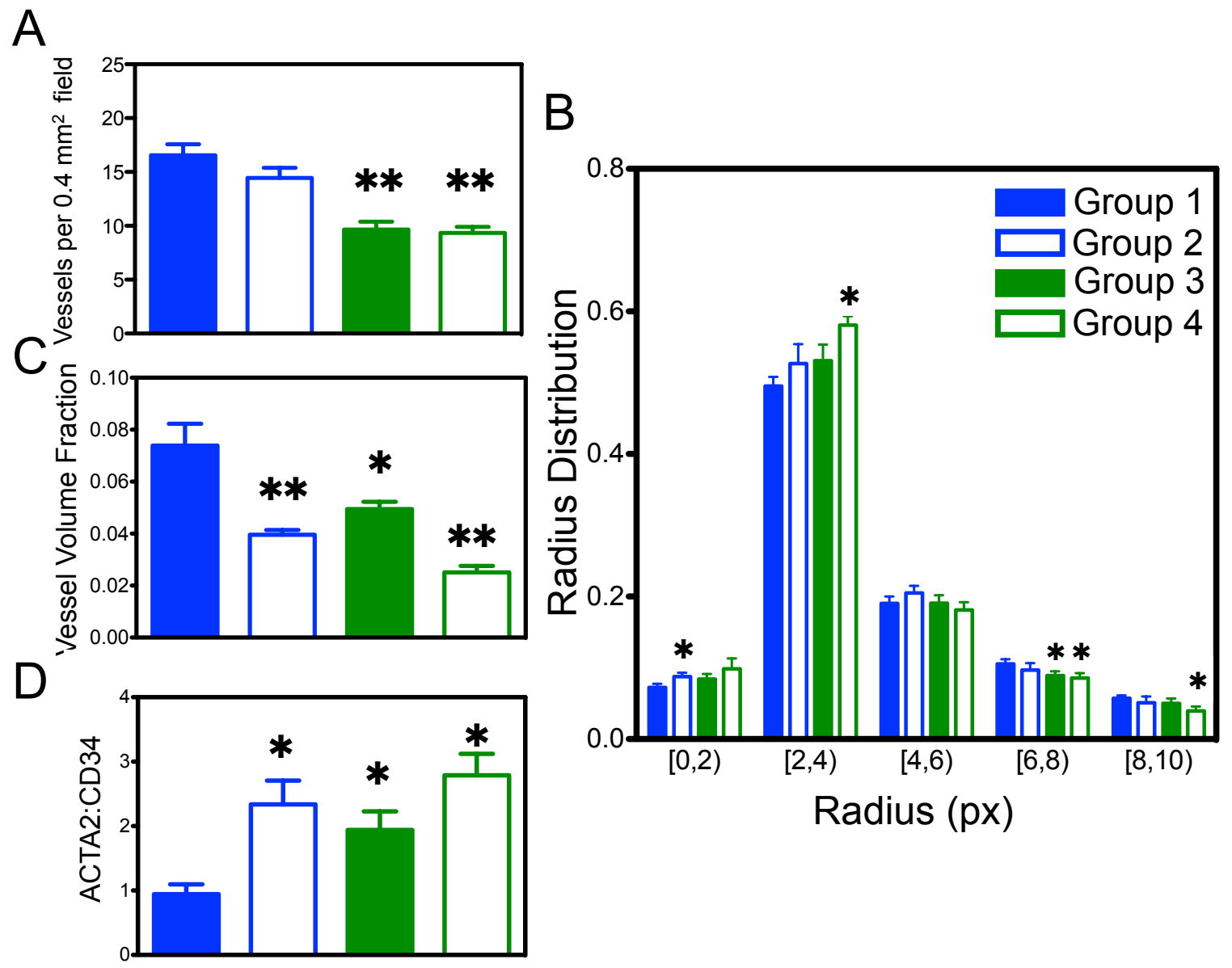

Figure 3.10 AV-951, in combination with hornerin siRNA therapy enhances antivessel effects. A. The number of CD34-positive vessels per $0.4 \mathrm{~mm}^{2}$ field was quantified in 20 representative $5 \mu \mathrm{m}$ sections. (4 tumors in each treatment group, 5 images per tumor). 12 thick section Z-stacks ( $50 \mu \mathrm{m}$ sections) from each group ( 3 fields per tumor, 4 tumors in each group) were analyzed via RAVE for B. radius distribution and C. vessel volume fraction. D. Ratiometric ACTA2:CD34 quantification of 12 images from each group (4 tumors per group, 3 sections per tumor) was measured by dividing the number of ACTA2 positive pixels by CD34-positive pixels. All data are represented by mean \pm s.e.m. $* \mathrm{p}<0.05, * * \mathrm{p}<0.005, * * * \mathrm{p}<0.0005$ one-tailed, unpaired student's t-test compared to control or Group 1. 
shown in Figure 3.8A. To confirm efficacy, tumors were measured after 5 days of AV951 treatment. As previously shown, AV-951 treated tumor volume decreased (156) and was 2.3-fold less than control (Figure 3.8B). For the combination experiments, the treatment groups are indicated in Figure 3.8C. At the termination of the experiment, tumor volume was measured for each treatment group (Figure 3.8D) via caliper measurement. Monotherapy (Groups 2 and 3) of either AV-951 or Hrnr siRNA resulted in 1.5 and 2.1-fold decreases in tumor burden compared to control Group 1, respectively. Combination treatment (Group 4) resulted in a 4.3-fold decrease in volume compared to controlGroup 1, resulting in an additive effect.

The vessels from each treatment group were morphologically distinct (Figure 3.9). Control, Group 1, tumors have typical tumor vasculature - numerous, large, irregular vessels. As expected, Group 2 (Hrnr siRNA) treatment resulted in vessels with less tumor vessel-like morphology, but no change in the number of vessels per field (Figure 3.10A). Again as expected, AV-951 (Group 3) vessels were morphologically similar vessels to Group 1 vessels; however, significantly fewer vessels were observed (Figure 3.10A), in keeping with previously published results (156). Interestingly, the combination therapy of hornerin siRNA and AV-951 (Group 4) resulted in vessels that were a hybrid between each individual treatment - less vessels were present (Figure 3.10A) in addition to morphologic differences. Quantitation with RAVE revealed the presence of an overall shift to smaller radii in Groups 2-4 compared to Group 1 (Figure 3.10B). In Groups 2-4, VVF was decreased compared to Group 1 (Figure 3.10C). To determine if pericyte recruitment is increased as a result of combination therapy, CD34:ACTA2 rati- 
ometric analysis was completed (Figure 3.10D). As in Figure 3.5, the increase in ACTA2 coverage after treatment with hornerin siRNA was 2.5-fold. Treatment with AV-951 alone resulted in a 2-fold increase. The additive deleterious effects on tumors and the differences in vessel morphology observed after treatment suggest the existence of compensatory pathways that should be accounted for when designing an anti-vascular cancer therapy.

\section{$\underline{3.4 \text { Materials and Methods }}$}

\subsubsection{Tumor Implantation}

For tumor implantation, cells were allowed to grow under normal culture conditions for 72 hours before being lifted with trypsin and suspended in aliquots of 500,000

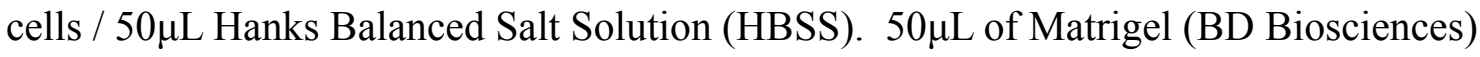
was added to each aliquot of cell suspension just before implantation (1:1 ratio). $100 \mu \mathrm{L}$ of Cell-Matrigel slurry was injected subcutaneously on the flanks of athymic nude mice and the injection site was monitored daily for growth by caliper.

\subsection{2 siRNA injections}

Silencer Select mouse hornerin (Hrnr) siRNA specific to mouse tissue and Silencer Select Negative Control \#1 Scrambled (scr) siRNA were purchased from Life Technologies. The siRNA sequence used in these experiments was aligned against human 
hornerin transcripts using the BLAST algorithm and no match was returned. $10 \mu \mathrm{g}$ of hornerin or scrambed siRNA were combined with in vivo-jetPEI transfection reagent (Polyplus, Illkirch, France) in solution with 5\% glucose per Polyplus instructions - total volume: $50 \mu \mathrm{L}$. Solutions were incubated for 30 minutes to allow formation of PEI-DNA complexes. After 30 minutes, siRNA solutions were injected slowly directly into the center of the tumor.

3.4.3 Tumor volume measurement, immunofluorescence and vessel parameter quantification.

The width and length of each tumor was measured by caliper. Tumor volume was calculated using the formula (width ${ }^{2} \mathrm{x}$ length)/2, where width was the length of the minor axis, while length was the length of the major axis.

At the end of the siRNA-only and siRNA+VEGF inhibitor studies, mice were sacrificed, cardiac perfused, and then tumors were excised and fixed in formalin overnight. Tumors were paraffin embedded and cut into $5 \mu \mathrm{m}$ sections (thin) or $50 \mu \mathrm{m}$ (thick/whole mount). After deparaffinization and antigen retrieval by standard histologic procedures, each section was blocked for 30 minutes in 3\% BSA in PBS (blocking buffer), incubated with anti-CD34 (1:100, Abcam, ab8158) in blocking buffer overnight at $4{ }^{\circ} \mathrm{C}$, before being incubated with goat anti-rat FITC-labeled secondary antibody (1:100, Abcam, ab6840) in blocking buffer. Slides were mounted with Prolong Gold Antifade reagent (Invitrogen). If assaying for alpha-smooth muscle action (Cy3-ACTA2, 1:200, Sigma, C6198), antibody was added along with the primary antibody. To obtain images for ves- 
sel parameter measurements, confocal Z-stack images $(50 \mu \mathrm{m})$ were constructed. Vessel parameters were measured using RAVE software previously developed in our lab (148).

\subsubsection{AV-951 administration}

AV-951 (Selleck Chemicals, Houston, Texas) was suspended in $0.5 \%$ methocel + $0.5 \%$ Tween-20 in HBSS to increase viscosity. Suspensions were sonicated to ensure even distribution of AV-951. $50 \mu \mathrm{L}$ of $1.0 \mathrm{mg} / \mathrm{kg}$ AV-951 or $0.0 \mathrm{mg} / \mathrm{kg}$ (control) were administered once daily by oral gavage through a 22 -gauge gavage needle (Kent Scientific, FNS-22-1.5, Torrington, Connecticut) connected to a $1 \mathrm{~mL}$ syringe.

\subsubsection{Microscopy}

\section{Epifluorescence:}

Olympus (Center Valley, Pennsylvannia) BX-41 microscope, equipped with a U-RFL-T high-powered Olympus mercury burner and a QImaging (Surrey, BC, Canada) Retiga2000R camera.

\section{Confocal:}

Nikon TE 2000-E2, equipped with a Melles Griot Argon Ion Laser System and Nikon DEclipse C1 accessories (Nikon Instruments, Melville, NY) using a 60X or 20X/1.45 NA oil-immersion objective and EZ-C1 software (Nikon Instruments). 


\subsubsection{DCE-MRI and $\mathrm{K}_{\text {trans }}$}

To assess the in vivo function of tumor vascularity, dynamic contrast-enhanced magnetic resonance imaging (DCE-MRI) was performed on a 7.0T Clinscan small animal imaging system (Bruker, Ettlingen, Germany) using a $30 \mathrm{~mm}$ inner-diameter birdcage radiofrequency coil. Nude mice $(\mathrm{n}=7)$ were imaged prior to treating tumors with Hrnr and Scr siRNA and again 6 days after treatment. Mice were anesthetized with $1.25 \%$ isoflurane anesthesia, which was maintained during imaging. Respiration and ECG were monitored during imaging using an MRI-compatible system (SA Instruments, Stony Brook, NY). An indwelling tail vein catheter was established for administration of the gadolinium contrast agent Gd-DTPA, which is required in DCE imaging. Mice were placed supine in the imaging system, and body temperature was maintained at $36^{\circ} \mathrm{C} \pm 1{ }^{\circ} \mathrm{C}$ by using circulating thermostated water. Tumors were localized using a spin-echo imaging sequence and an imaging plane that transected both tumors was chosen for DCE imaging.

A dual-Gd-bolus DCE-MRI acquisition strategy (158)was employed, which uses a low dose of contrast agent to accurately acquire the undistorted Gd-DTPA concentration vs. time curve in the blood pool and a higher dose of contrast agent to acquire the GdDTPA concentration vs. time curve in the tumor with high signal-to-noise ratio, since GdDTPA is known to have signal saturation effects at high doses in the blood pool (158). Short axis ECG-gated images of the left ventricle were acquired using a saturationrecovery gradient echo pulse sequence with the following imaging parameters: number of images $=500,1$ image per heartbeat, echo time/repetition time/saturation delay time $=$ 
$0.8 / 1.6 / 10 \mathrm{~ms}$, excitation flip angle $=25^{\circ}$, image resolution $=0.47 \times 0.47 \times 1 \mathrm{~mm}$, field of view $=30 \mathrm{~mm}$, Gd-DTPA dose $=0.025 \mathrm{mmol} / \mathrm{kg}$, imaging time $\sim 1$ minute. ECG-andrespiratory gated images of the tumors were similarly acquired using a saturationrecovery gradient echo pulse sequence with the following imaging parameters: number of images $=350$, echo time $/$ repetition time/saturation delay time $=1.1 / 2.0 / 200 \mathrm{~ms}$, excitation flip angle $=25^{\circ}$, averages $=2$, image resolution $=0.195 \times 0.195 \times 1.5 \mathrm{~mm}$, field of view $=25 \mathrm{~mm}$, Gd-DTPA dose $=0.15 \mathrm{mmol} / \mathrm{kg}$, imaging time $\sim 5$ minutes. Gd-DTPA concentration vs. time curves were generated by drawing regions of interest around the left ventricular cavity and each tumor. Tracer kinetic Kety model analysis (159) was applied to the left ventricular and tumor Gd-DTPA concentration-vs-time curves, which yielded an estimate of the volume transfer constant $\mathrm{K}_{\text {trans, }}$, a widely-accepted MRI measurement of vascularity(160-162). 
CHAPTER 4: HORNERIN EXPRESSION CONFERS RESISTANCE TO OXIDATIVE STRESS THROUGH ACTIVATION OF NF-kB 


\section{$\underline{4.1 \text { Abstract }}$}

The knockdown of hornerin via intratumoral injections resulted in vessels with drastically different morphology. Knockdown tumors were characterized by significantly decreased VVF, fractal dimension and vessel radii. Interestingly, knockdown of hornerin expression did not result in a significant change in the number of tumor vessels. Taken together, these facts led us to investigate hornerin as an endothelial survival mediator.

To measure the survival benefits of hornerin expression, transient transfection was needed, since hornerin expression is low or non-detectable by traditional western blotting. A codon-optimized synthetic gene was engineered for easy transfer between expression vectors. We chose to express a hornerin-EGFP fusion protein for subsequent assays and easy identification of hornerin expressing cells. Hydrogen peroxide and other free radicals are present in the tumor milieu and are known to cause cell death. We hypothesized that hornerin could provide protection from these insults. Therefore, we subjected hornerin-expressing HUVECs to oxidative stress by incubation with hydrogen peroxide and observed decreased cell death as measured by terminal deoxynucleotidyl transferase (TdT)-mediated dUTP nick-end labeling (TUNEL) staining. An examination of transfected cells revealed activated NF- $\mathrm{BB}$ only in the hornerin-positive cells; non-transfected and control cells displayed cytoplasmic or non-activated NF- $\kappa$ B. Further, NF- $\kappa$ B activation and nuclear translocation increased BCL-2 (anti-apoptotic protein) localization to the mitochondria. Pharmacologic inhibition of NF- $\kappa$ B translocation by JSH-23 completely eliminated the survival benefit in hornerin-expressing cells. The discovery of this 
hornerin-mediated protective effect provides additional avenues to explore when designing hornerin-based, anti-tumor vascular strategies.

\subsection{Introduction}

Careful examination of whole mounted tumor vessels from hornerin siRNA treated tumors revealed differences in vessel morphology without significant differences in the number of vessels per imaging field. This finding led us to investigate the potential for hornerin to confer survival benefits to tumor vessels. Tumor vessels must proliferate; but they also must survive their harsh surroundings. The tumor microenvironment contains a milieu of growth factors, cells, and extracellular matrix. Several cell types within the tumor microenvironment are known to produce reactive oxygen species (ROS) such as hydrogen peroxide $\left(\mathrm{H}_{2} \mathrm{O}_{2}\right)$. These include tumor cells $(163,164)$ and tumor infiltrating myeloid cells, such as macrophages, granulocytes, and dendritic cells (165). The generation of hydrogen peroxide has been reported to be in the range of $0.2-0.5 \mathrm{nmol} / 10^{4}$ cells/hr in vitro (166). At this rate, local hydrogen peroxide concentrations in the tumor environment could quickly build to mid-micromolar levels. While at low concentrations (nanomolar to low-micromolar), hydrogen peroxide is reported to increase cell proliferation (167) through activation of downstream signaling cascades, high (around $10^{-4} \mathrm{M}$ ) hydrogen peroxide concentration induces apoptosis in HUVECs $(168,169)$. We hypothesized that hornerin might confer a survival advantage to endothelial cells when challenged with hydrogen peroxide. Indeed, when subjected to oxidative stress via hydrogen peroxide, EGFP-HRNR expressing HUVECs were more resistant to death than trans- 
fected (EGFP) and non-transfected controls. We believe this protective effect in hornerin expressing HUVECs is mediated by NF- $\mathrm{kB}$ activation and subsequent BCL-2 localization to the mitochondria. Constitutive NF- $\mathrm{kB}$ activation is a hallmark of a variety of solid tumors, including breast, colon, prostate, and pancreas among many others (170). Constitutive activation is thought to contribute to chemoresistance (171). Once activated NF- $\mathrm{kB}$ translocates to the nucleus and host of regulatory genes are activated. BCL-2 and other apoptotic genes are included in the list of regulatory genes activated by NF-KB. BCL-2 also contributes to chemoresistance $(172,173)$. Further, the ratio of pro-apoptotic protein BAX to anti-apoptotic BCL-2 has been used to predict susceptibility to apoptosis induced by cytotoxic agents $(173,174)$. We observed an increase in NF- $\mathrm{kB}$ activation and BCL2/mitochondrial colocalization in hornerin expressing HUVECs and corresponding protection from oxidative stress induced cell death. When activation of NF-kB was inhibited by incubation with JSH-23 (inhibitor of NF-kB nuclear translocation (175)), BCL-2/ mitochondrial colocalization decreased and hornerin expressing cells were no longer protected from cell death. The description of the mechanism of hornerin induced NF- $\mathrm{KB}$ activation could potentially direct the creation of molecularly targeted hornerin antivascular therapies specific to NF-kB activation, providing an additional route of therapeutic intervention.

\subsection{Results and Discussion}


4.3.1 EGFP-hornerin fusion protein exhibits a similar expression pattern compared to TCM-treated HUVECs

To produce EGFP-hornerin (EGFP-HRNR) fusion protein, a DNA construct including a modified hornerin gene complete with common restriction sites was engineered. The hornerin gene was modified via codon-optimization methods established by Genscript USA, Inc. to improve mammalian cell expression. The full length engineered DNA construct that drives hornerin expression is shown in Figure 4.1A. For the creation of an EGFP-HRNR fusion protein, full length engineered gene (Figure 4.1A) was SmaI restriction digested (Figure 4.1B) and ligated into an appropriate EGFP vector. HUVECs were transfected with a control EGFP construct or hornerin-EGFP construct. Typical electroporation transfection efficiency measured by flow cytometry is shown in Figure 4.1C. EGFP is diffusely expressed in control transfected HUVECs (Figure 4.1D). Punctate, perinuclear expressed HRNR-EGFP (Figure 4.1E) matches the localization of immunofluorescently detected hornerin in TCM treated HUVECs (Figure 4.1F). Once expression conditions were optimized, cells were investigated for their susceptibility to acute oxidative stress.

4.3.2 Hornerin expression confers resistance to oxidative stress

Transfected HUVECs were subjected to oxidative stress via acute treatment with $600 \mu \mathrm{M}$ hydrogen peroxide. Cell death was assessed by terminal deoxynucleotidyl transferase (TdT)-mediated dUTP nick-end labeling (TUNEL) analysis. Vehicle (PBS) treat- 

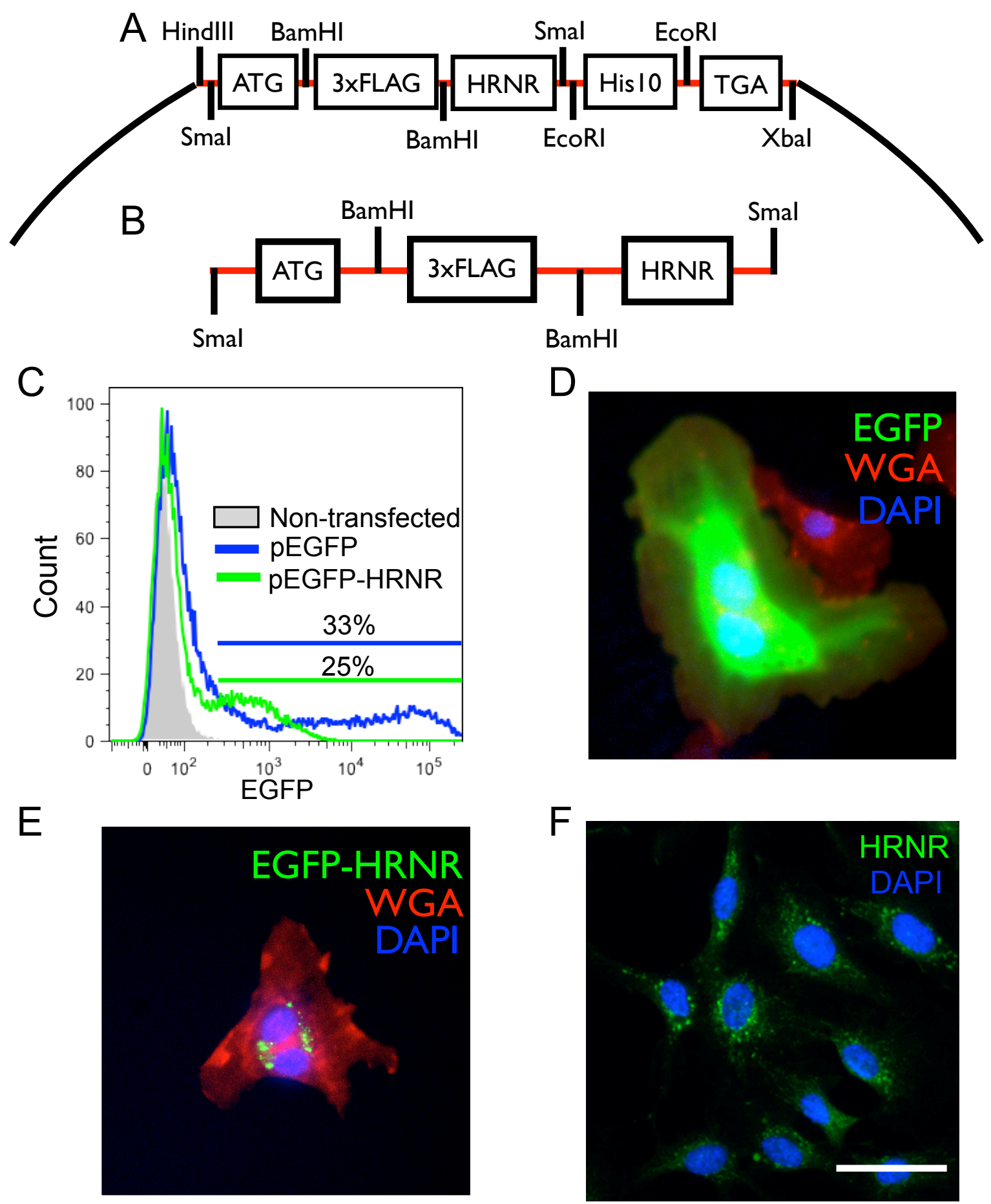

Figure 4.1 Engineered EGFP-HRNR expression matches TCM-induced HUVEC expression. A. Engineered DNA construct including HRNR gene, 3X FLAG and His 10 affinity tags, and HindIII, Sma I, BamHI, EcoRI, XbaI labeled restriction sites. B. Construct from A. modified for insertion into pEGFP-N2 plasmid to form EGFP-HRNR fusion protein. C. Flow cytometry data showing typical transfection efficiencies. D. EGFP is diffusely expressed in HUVECs, while EGFP-HRNR expression is punctate and perinuclear. Wheat germ aggulutinin (WGA) and DAPI used as cell counterstain. F. TCMtreated HUVECs display similar hornerin expression pattern. Scale bar: $50 \mu \mathrm{m}$. 

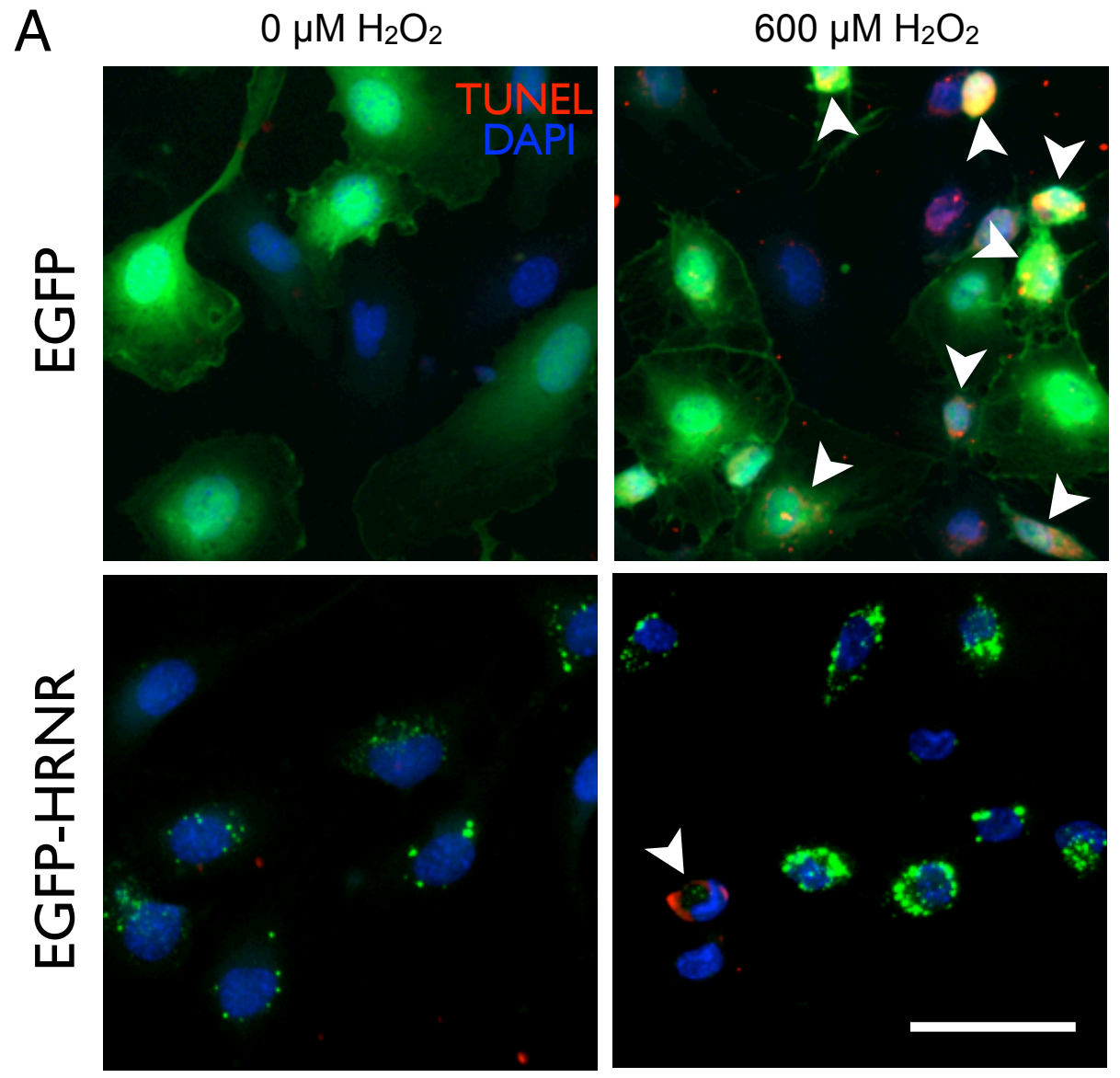

B

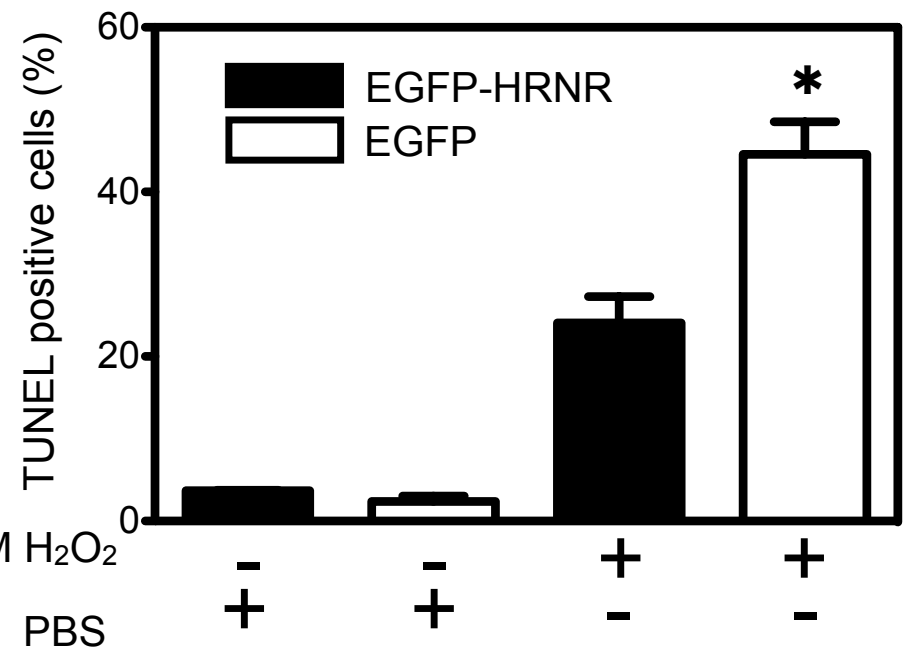

Figure 4.2 EGFP-HRNR cells protected from oxidative stress. A. Representative images of TUNEL stained EGFP and EGFP-HRNR cells treated with PBS or $600 \mu \mathrm{M} \mathrm{H}_{2} \mathrm{O}_{2}$ for 4 hours. Arrowheads highlight TUNEL-positive transfected cells. DAPI used as nuclear counterstain. B. TUNEL analysis on EGFP and EGFP-HRNR cells. Cells were treated with PBS or $600 \mu \mathrm{M}$ hydrogen peroxide for 4 hours and assessed for TUNEL positivity. Data representative of 3 separate experiments, $520 \mathrm{X}$ image panes per experiment. Data represented as mean \pm s.e.m. $* \mathrm{p}<0.05$. Scale bar: $50 \mu \mathrm{m}$. 

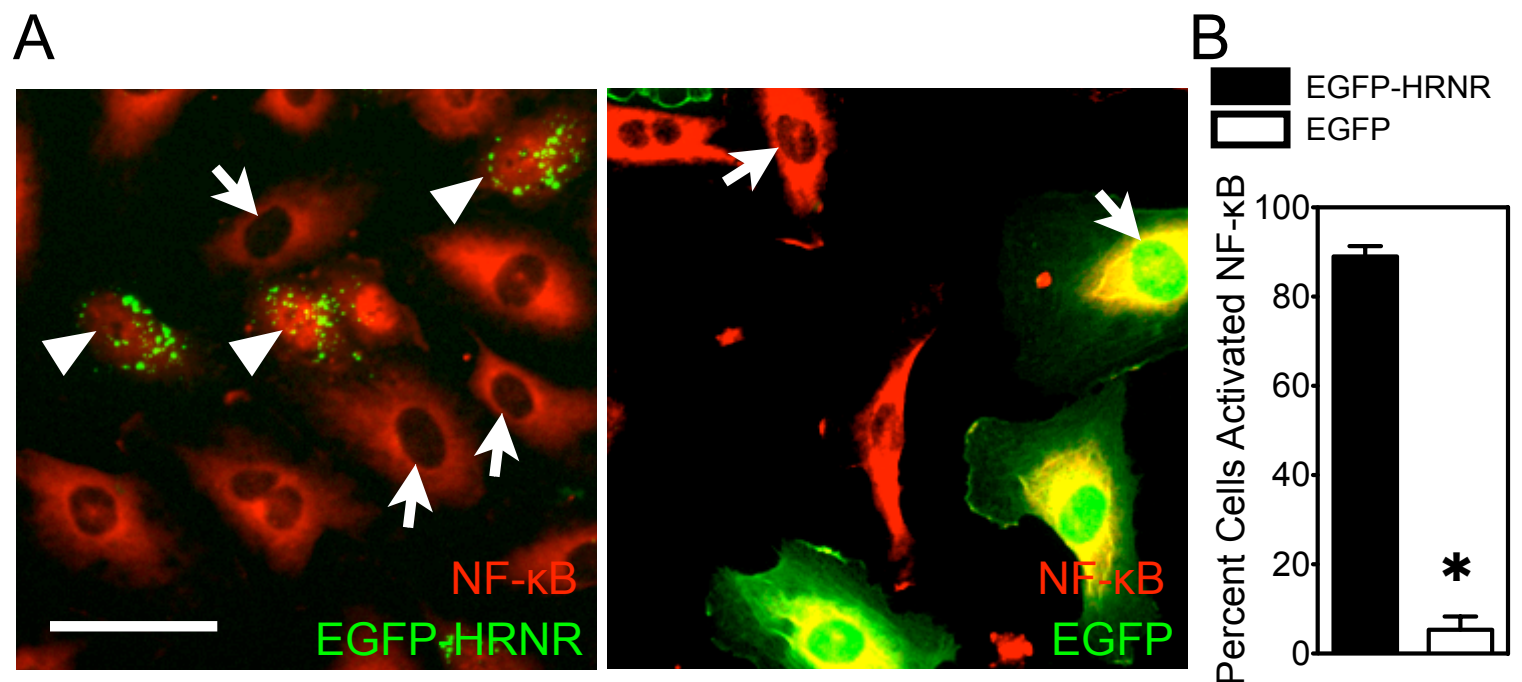

\section{C}
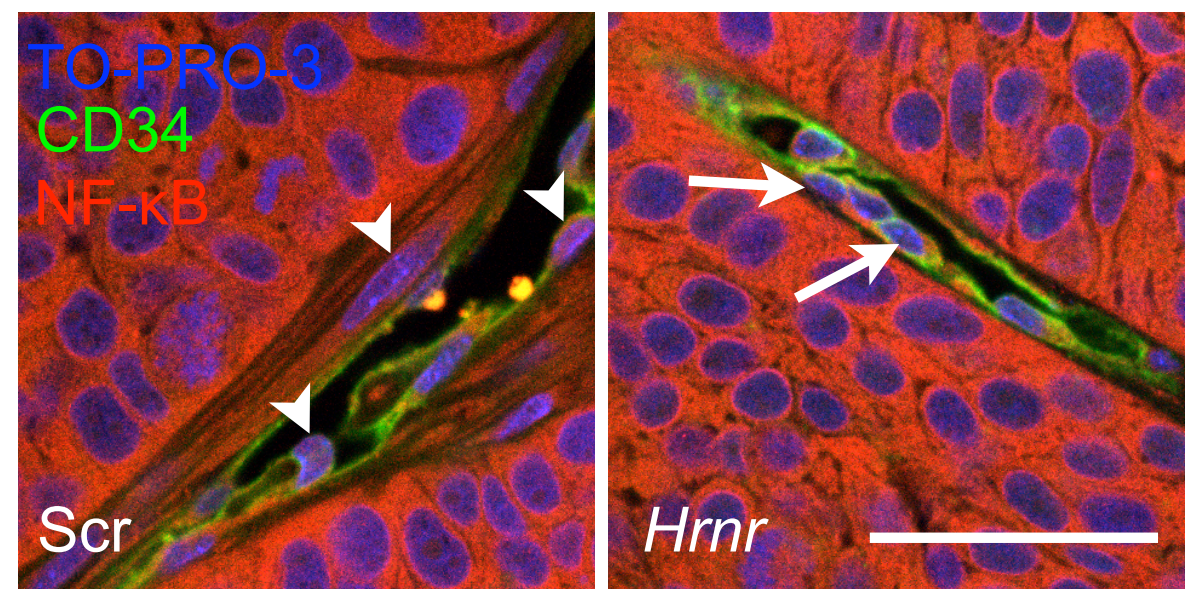

Figure 4.3 Hornerin expression induces NF- $x \mathrm{~B}$ activation and nuclear translocation. A. Epifluorescent immunofluorescent staining of RELA (subunit of NF- $\kappa B$ ) in EGFPHRNR (left) and EGFP control (right) cells. Non-activated cytoplasmic localizaton is indicated by arrows; activated nuclear localization indicated by arrowheads. B. Quantification of nuclear NF- $\kappa \mathrm{B}$ translocation in three separate experiments. Cells from $1020 \mathrm{X}$ images were used for quantification. C. Hrnr knockdown results in non-activated, cytoplasmic NF- $x$ B expression in endothelial cells (CD34-positive); however, activated, nuclear (TO-PRO-3) NF- $x$ B was present in Scr treated tumors. Nuclear NF- $x$ B is highlighted by arrows, cytoplasmic NF- $\varkappa \mathrm{B}$ is highlighted by arrowheads. Data represented as mean \pm s.e.m. $* \mathrm{p}<0.05$. Scale bars: $50 \mu \mathrm{m}$. 
ment resulted in very few TUNEL positive EGFP and EGFP-HRNR cells (Figure 4.2A) Quantification of three separate experiments yielded mean TUNEL positive rates of 3.7 and $2.4 \%$, respectively (Figure 4.2B). Exposure to $\mathrm{H}_{2} \mathrm{O}_{2}$ increased TUNEL-positive control EGFP cells from 2.4 to $44.6 \%$ (Figure $4.2 \mathrm{~A}$ and B), percentages similar to previously published results (176). In contrast, treatment of EGFP-HRNR transfected cells with $\mathrm{H}_{2} \mathrm{O}_{2}$ resulted in an increase from 3.7 to only $24.1 \%$ TUNEL-positive cells (Figure $4.2 \mathrm{~A}$ and B). Comparing TUNEL-positivity in EGFP and EGFP-HRNR cells exposed to $\mathrm{H}_{2} \mathrm{O}_{2}$, EGFP-HRNR cells resulted in significant protection, 1.9-fold decreased cell death (Figure 4.2A and $\mathrm{B}, 600 \mu \mathrm{M} \mathrm{H}_{2} \mathrm{O}_{2}$ column), indicating a survival benefit of cells expressing hornerin.

4.3.3 Hornerin expression results in activation of NF- $\kappa$ B and subsequent BCL-2 localization to mitochondria

Constitutive NF- $\kappa$ B activation is required for resistance to hydrogen peroxide induced apoptosis in hepatocellular cancer cells (176), so we investigated whether NF-кB plays a role in hornerin mediated survival. Immunofluorescent interrogation of $\mathrm{NF}-\kappa \mathrm{B}$

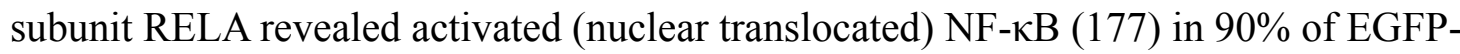
HRNR cells (Figure 4.3A, left and B), while only 5\% of EGFP cells contained activated NF- $\kappa \mathrm{B}$ (Figure 4.3A, right and B). To confirm and further support this observation, we

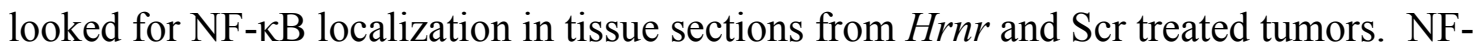
$\kappa \mathrm{B}$ expression was mostly cytoplasmic in tumor vessels treated with Hrnr siRNA, whereas nuclear, activated NF- $\kappa \mathrm{B}$ is observed in Scr siRNA injected tumors (Figure 

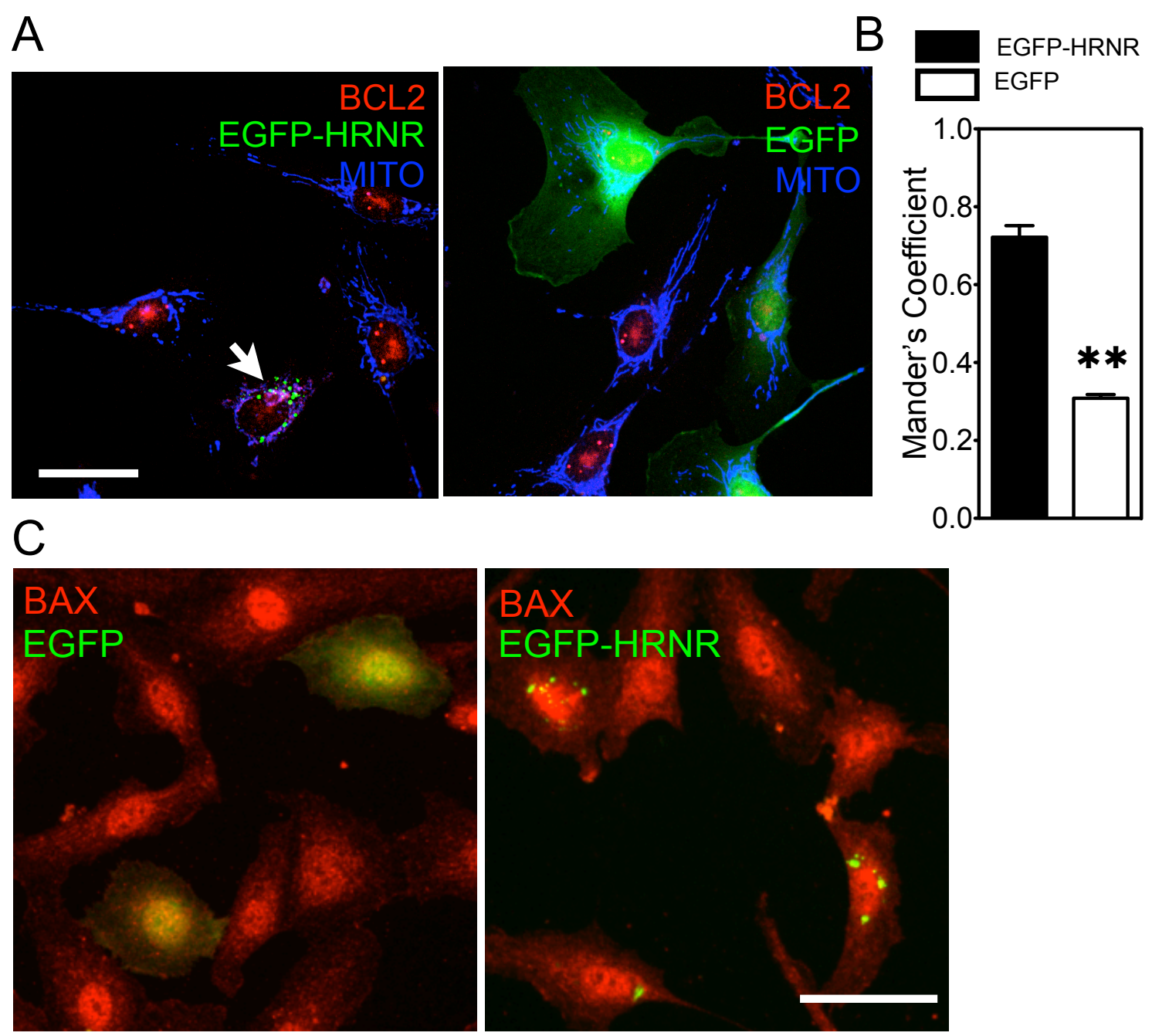

Figure 4.4 Hornerin expression induces BCL-2/mitochondrial localization. A. Confocal images of BCL2/mitochondria (mitotracker dye, "MITO") localization in EGFPHRNR (left) and EGFP control (right) cells. Arrow indicates strong colocalization. B. Quantification of colocalization via Mander's coefficient from three separate experiments. Cells from at least $2060 \mathrm{X}$ images were used for quantification. C. EGFP and EGFP-HRNR expressing cells show no apparent difference in BAX protein expression. ** $\mathrm{p}<0.005$. Scale bar: $50 \mu \mathrm{m}$. 
A
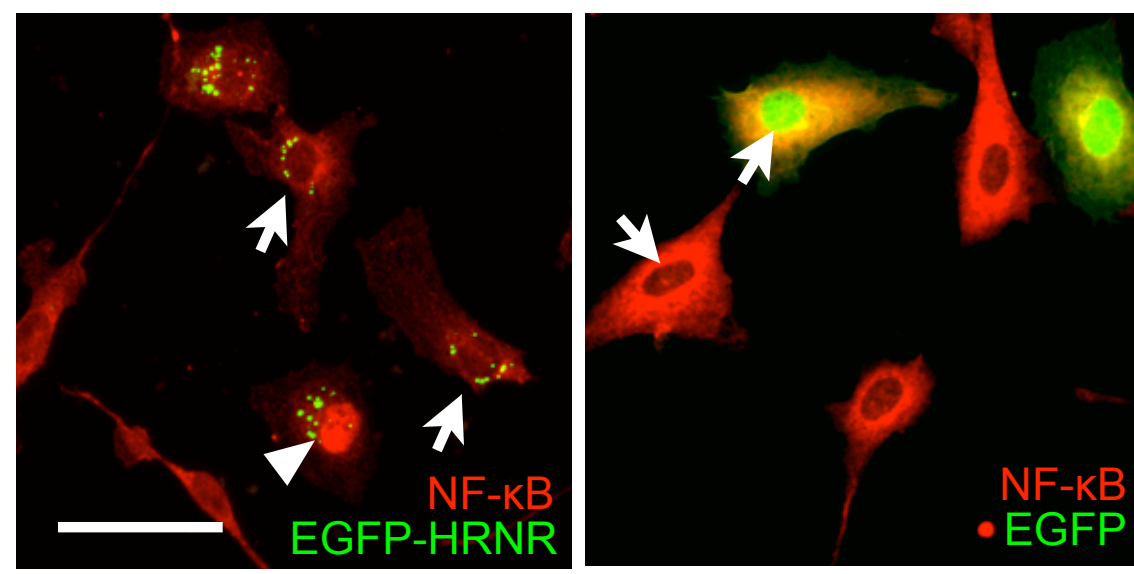

C

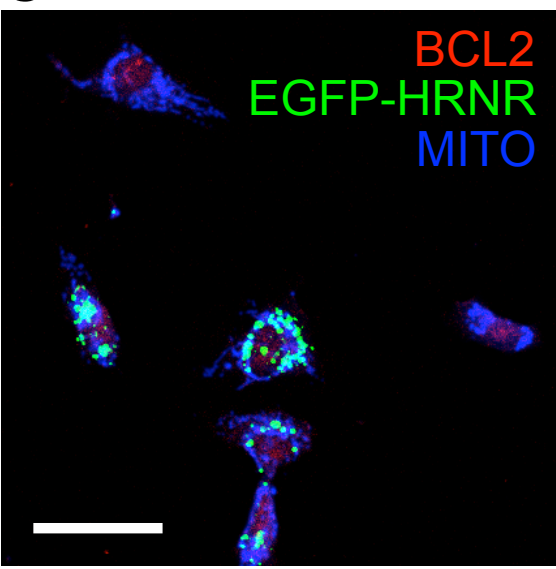

- EGFP

B

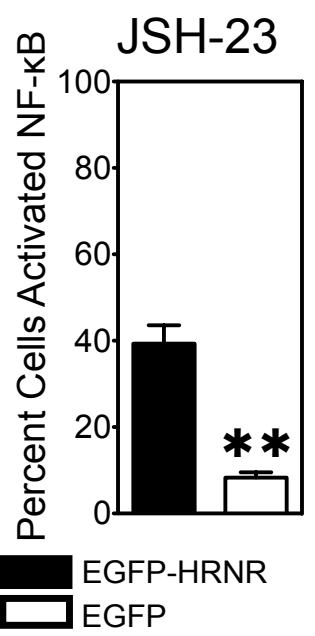

$\mathrm{D}$
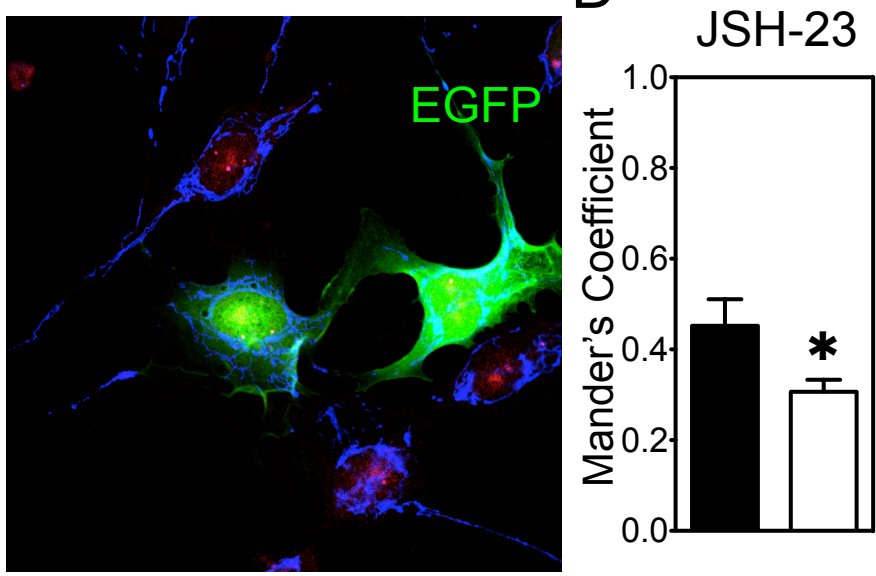

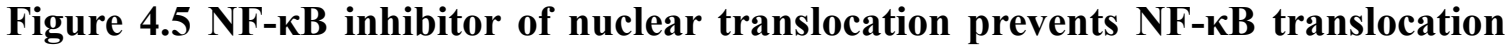
and disrupts BCL-2/mitochondrial localization. A. Epifluorescent immunofluorescent staining of RELA (subunit of NF- $\kappa \mathrm{B}$ ) in EGFP-HRNR (left) and EGFP control (right) cells after incubation with $25 \mu \mathrm{M}$ JSH-23 for 12 hours prior to staining. Non-activated cytoplasmic localizaton is indicated by arrows; activated nuclear localization indicated by arrowheads B. Quantification of nuclear NF- $\kappa$ B translocation in three separate experiments. Cells from $1020 \mathrm{X}$ images were used for quantification. C. Confocal images of BCL2/mitochondria (mitotracker dye, "MITO") localization in EGFP-HRNR (left) and EGFP control (right) cells after incubation with $25 \mu \mathrm{M}$ JSH-23 for 12 hours prior to staining.. D. Quantification of colocalization via Mander's coefficient from three separate experiments. Cells from at least $2060 \mathrm{X}$ images were used for quantification. Scale bars: $50 \mu \mathrm{m} . * \mathrm{p}<0.05, * * \mathrm{p}<0.005$. 
A

$25 \mu \mathrm{M}$ JSH-23

$0 \mu \mathrm{M} \mathrm{H}_{2} \mathrm{O}_{2}$
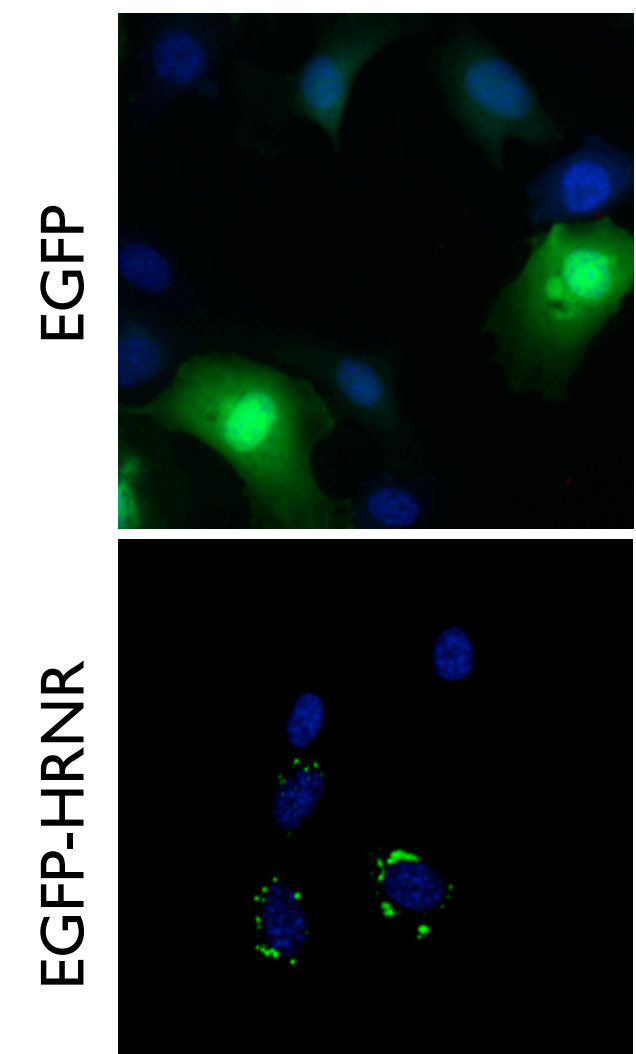

B

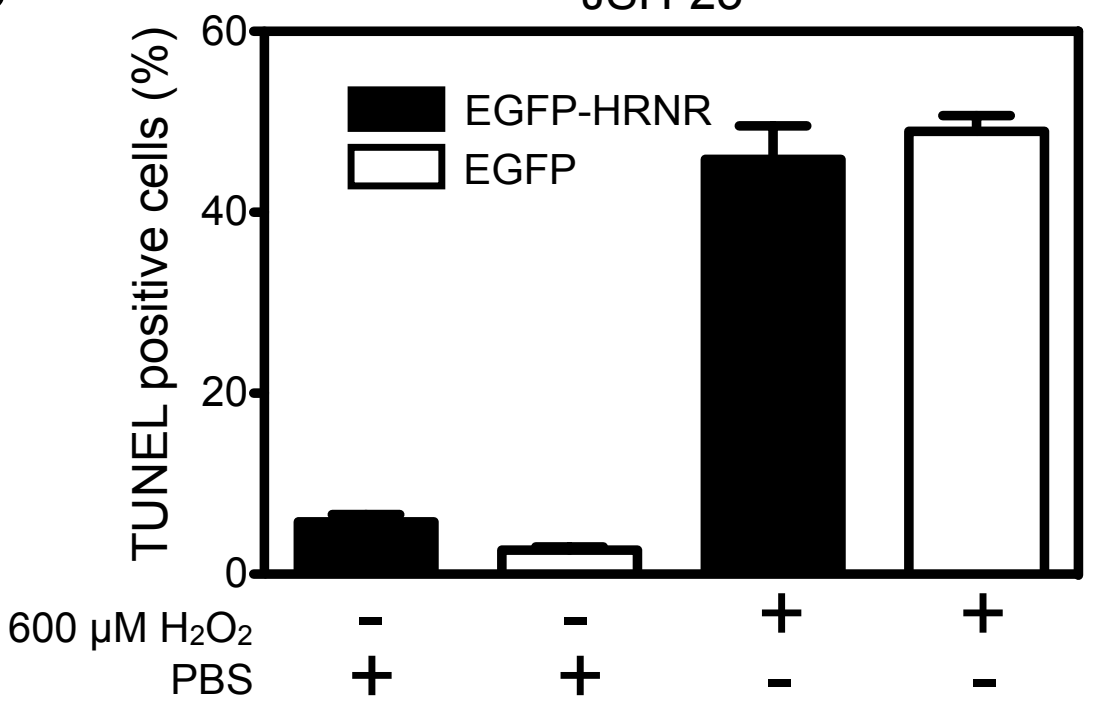

$25 \mu \mathrm{M} \mathrm{JSH}-23$

$600 \mu \mathrm{M} \mathrm{H}_{2} \mathrm{O}_{2}$
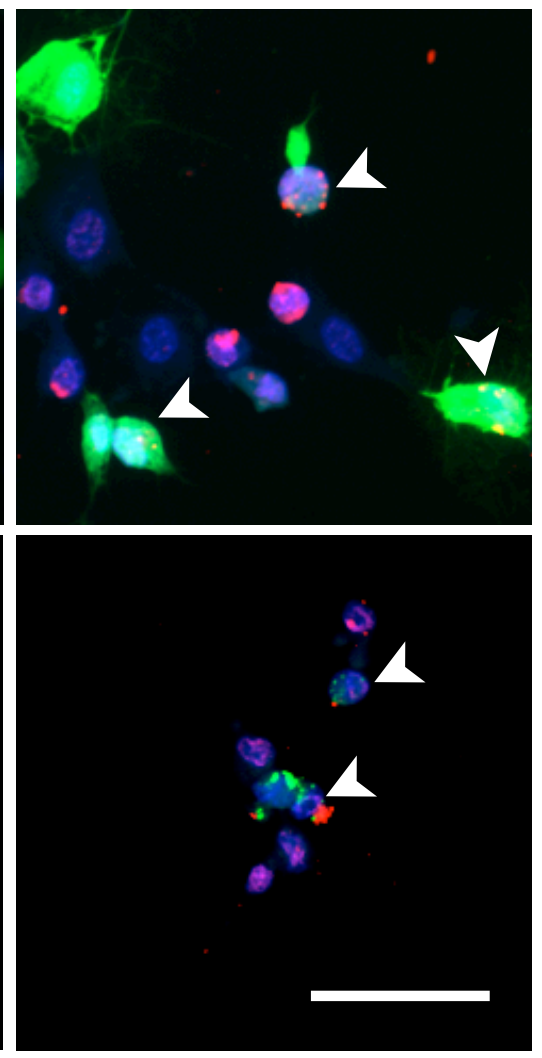

JSH-23 
$4.3 \mathrm{C})$.

$\mathrm{NF}-\kappa \mathrm{B}$ regulates genes involved in apoptosis (178) such as anti-apoptotic BCL2 (179). Therefore, we stained HUVECs for BCL2 expression and observed that EGFPHRNR positive cells displayed mitochondrially localized BCL2 expression (Figure 4.4A, left) where BCL2 exerts its anti-apoptotic activity $(180,181)$. BCL2/mitochondrial localization in EGFP cells (Figure 4.4A, right). To quantify colocalization resulting from hornerin expression, we used Mander's coefficient, a measure of colocalization that was created to alleviate some of the difficulties interpreting Pearson's correlation coefficient (182). BCL2/mitochondrial colocalization as measured by Mander's coefficient was significantly increased in EGFP-HRNR cells (Figure 4.4B). Probing another protein important in apoptosis, we stained for BAX (pro-apoptotic protein). Unlike BCL2, there was no difference in BAX staining between EGFP-HRNR and EGFP cells (Figure 4.4C).

4.3.4 Incubation with JSH-23, an inhibitor of NF- $\mathrm{BB}$ nuclear translocation reduces NF$\kappa \mathrm{B}$ translocation, $\mathrm{BCL}-2 /$ mitochondria localization and protection from oxidative stress in hornerin expressing cells.

Finally, we were able to mitigate hornerin-mediated effects by incubation of EGFP-HRNR transfected cells with JSH-23, an inhibitor that prevents nuclear translocation without affecting $\mathrm{I} \kappa \mathrm{B} \alpha$ degradation $\mathrm{NF}-\kappa \mathrm{B}$ (175). Incubation with JSH-23 partially reversed nuclear translocation of NF- $\mathrm{KB}$ in EGFP-HRNR cells from 89 (uninhibited, Figure $4.3 \mathrm{~B}$ ) to $39 \%$ (Figure $4.5 \mathrm{~A}$ and $\mathrm{B}$ ) and mitochondrial colocalization of BCL2 (Figure 
4.5C and D) from 0.72 (uninhibited, Figure 4.4B) to 0.45. Additionally, pre-incubation with JSH-23 eliminated the survival advantage during oxidative stress. Representative images used for TUNEL quantification after treatment with JSH-23 are shown in Figure 4.6A. After inhibition with JSH-23, incubation with $\mathrm{H}_{2} \mathrm{O}_{2}$ resulted in 45.9 and $49.0 \%$ TUNEL-positive cells in EGFP-HRNR and EGFP HUVECs, respectively (Figure 4.6B).

\subsection{Materials and Methods}

\subsubsection{Recombinant Hornerin Expression}

A codon optimized, full-length human hornerin gene was synthesized by Genscript USA using in-house procedures. A 3X FLAG-tag was added to the N-terminal and that engineered gene was inserted into pEGFP-N2 plasmid (Invitrogen) via SmaI restriction sites in the multiple cloning site (MCS). Control EGFP plasmid was unmodified pEGFP-N2. Transfection by electroporation was completed via Invitrogen's Neon Transfection system. Specifically, one pulse of $1350 \mathrm{~V}, 30 \mathrm{msec}$ in duration was used for the transfection. Transfected cells were grown in antibiotic free media for 12 hours to prevent cytotoxicity from antibiotic accumulation in porous cells. After 12 hours, normal growth medium was replaced and cells were cultured until the initiation of experiments.

4.4.2 Annotated sequence of engineered DNA construct supplied by Genscript

HindIII

AAGCTT

SmaI 
CCCGGG

Start

ATG

BamHI

GGATCC

$3 \times F l a g$

GACTACAAAGACGATGACGACAAGGGGTCAGGCGATTATAAGGATGACGACGAT

AAGGGTTCGGGAGACTATAAAGA

TGATGACGATAAAGGATCTGGT

BamHI

GGATCC

HRNR MRNA

ССТAAACTCCTACAAGGCGTCATCACTGTCATCGATGTTTTCTACCAATATGCC ACCCAGCATGGGGAGTATGATACGTTGAACAAGGCAGAGCTGAAAGAACTTCTG GAAAATGAGTTTCATCAAATTCTGAAGAATCCAAACGATCCAGATACTGTGGAT ATCATCTTGCAAAGTCTGGATCGAGACCATAACAAGAAAGTGGATTTTACTGAG TATCTTCTGATGATATTCAAGCTGGTTCAGGCTCGTAATAAAATCATTGGCAAA GATTACTGCCAAGTTTCAGGGTCAAAGCTGAGAGATGACACTCACCAGCACCAA GAGGAACAAGAAGAAACTGAAAAAGAGGAGAACAAACGGCAAGAATCCTCTTTT AGTCATTCAAGTTGGAGTGCAGGAGAGAATGATTCCTATTCCAGAAACGTCAGA GGAAGTCTTAAACCTGGGACTGAATCCATATCCAGAAGACTGAGTTTTCAAAGA GACTTTTCTGGCCAACATAACTCCTACTCAGGTCAGTCTTCCAGCTATGGTGAG CAAAACTCCGACTCCCATCAGTCTTCAGGCCGCGGCCAATGTGGGTCTGGGTCA GGGCAGTCTCCCAACTATGGCCAACACGGCTCTGGCTCCGGACAGTCTTCCAGC AATGACACACATGGGTCTGGCTCAGGCCAGTCTTCTGGCTTTAGTCAACACAAG TCTAGCTCAGGGCAGTCCTCTGGTTACAGTCAGCATGGATCTGGCTCAGGTCAC TCCTCTGGCTACGGACAACACGGCTCTAGGTCAGGACAGTCATCTAGGGGTGAA CGACACAGATCTAGCTCAGGTTCGTCTTCCAGCTATGGTCAGCATGGGTCTGGT TCCCGTCAGTCTTTGGGCCACGGCCGACAAGGGTCTGGATCTCGCCAGTCTCCT AGCCACGTCCGACATGGGTCCGGTTCGGGGCACTCCTCCAGCCACGGCCAACAC GGGTCTGGCTCAAGTTACTCTTACAGCCGTGGCCATTATGAGTCTGGCTCAGGC CAGACTTCTGGCTTTGGGCAACATGAGTCTGGCTCAGGACAGTCCTCTGGCTAT AGTAAGCATGGTTCTGGCTCAGGTCACTCCTCTAGCCAGGGACAACATGGATCT ACGTCAGGGCAGGCATCAAGCTCTGGCCAACATGGCTCCAGCTCACGTCAGTCT TCCAGCTATGGTCAGCATGAGTCTGCCTCCCGTCACTCTTCAGGCCGCGGCCAA CACAGCTCTGGATCTGGCCAGTCTCCAGGCCACGGCCAGCGTGGGTCTGGGTCA GGGCAGTCTCCCAGCTCCGGCCAACATGGGACTGGCTTTGGTCGATCTTCCAGC AGTGGCCCATATGTGTCTGGTTCAGGCTACTCTTCTGGCTTTGGTCACCACGAG TCTAGCTCAGAGCATTCCTCTGGTTACACTCAGCATGGATCTGGCTCAGGTCAC TCCTCCGGCCACGGACAACACGGCTCTAGGTCAGGACAGTCATCTAGGGGTGAA 
CGACAAGGATCTAGTGCAGGTTCATCTTCCAGCTATGGTCAGCATGGGTCTGGC TCCCGTCAATCTTTGGGACACAGCCGACATGGGTCTGGATCTGGCCAGTCTCCT AGCCCTAGCCGTGGCCGACATGAGTCTGGTTCCAGGCAGTCTTCCAGCTATGGC CCACATGGGTATGGCTCAGGGAGGTCTTCAAGCCGTGGCCCATATGAGTCTGGC TCCGGTCACTCTTCTGGCTTAGGTCACCAAGAGTCTCGCTCAGGACAGTCCTCT GGCTACGGTCAACACGGATCTAGCTCGGGTCATTCCTCTACCCATGGGCAACAT GGTTCTACATCAGGACAGTCATCGAGCTGTGGCCAACATGGAGCTACCTCAGGT CAGTCTTCCAGCCACGGTCAGCATGGCTCTGGCTCAAGTCAGTCTTCTCGCTAT GGCCAACAGGGCTCTGGATCTGGCCAGTCTCCTAGTCGCGGCCGACATGGGTCC GATTTTGGGCACTCTTCCAGCTACGGCCAACATGGGTCTGGCTCCGGTTGGTCT TCAAGCAATGGCCCACATGGGTCTGTCTCAGGCCAGTCTTCCGGCTTTGGTCAC AAGTCTGGCTCAGGGCAGTCCTCTGGTTACAGTCAGCATGGATCTGGCTCAAGT CACTCCTCCGGCTACAGAAAACACGGCTCTAGGTCAGGACAGTCATCTAGGAGT GAACAACACGGATCTAGCTCAGGTTTGTCTTCCAGCTATGGTCAGCATGGGTCG GGCTCCCATCAATCTTCGGGCCACGGCCGACAAGGGTCTGGATCTGGCCACTCT CCTAGCCGTGTCCGACATGGGTCCAGTTCAGGGCACTCCTCCAGCCACGGCCAA CACGGGTCTGGCACAAGTTGTTCTTCCAGCTGTGGCCATTATGAGTCTGGCTCA GGCCAGGCTTCTGGTTTTGGGCAACACGAGTCTGGCTCAGGACAGGGCTATAGT CAGCATGGTTCTGCCTCAGGTCACTTCTCTAGCCAGGGACGACATGGATCTACG TCAGGGCAGTCATCAAGCTCCGGCCAACATGACTCTAGCTCAGGTCAATCTTCC AGCTATGGTCAGCATGAGTCTGCCTCCCATCACGCTTCGGGCCGCGGCCGACAT GGCTCTGGATCTGGCCAGTCTCCAGGCCACGGCCAGCGTGGGTCTGGGTCAGGG CAGTCTCCCAGCTATGGCCGACATGGGTCTGGCTCCGGTCGGTCTTCCAGCAGT GGCCGACATGGGTCTGGCTCAGGCCAGTCTTCTGGCTTTGGTCACAAGTCTAGC TCAGGGCAGTCCTCTGGTTACACTCAGCATGGATCTGGCTCAGGTCACTCCTCC AGCTACGAACAACACGGCTCTAGGTCAGGACAGTCATCTAGGAGCGAACAACAT GGATCTAGCTCAGGTTCGTCTTCCAGCTATGGTCAGCATGGGTCTGGCTCCCGT CAGTCTTTGGGCCACGGCCAACATGGGTCTGGATCTGGCCAGTCTCCTAGCCCT AGCCGTGGCCGACATGGGTCTGGTTCCGGGCAGTCTTCCAGCTATGGCCCATAT AGGTCTGGCTCAGGGTGGTCTTCAAGCCGTGGCCCATATGAGTCTGGCTCCGGT CACTCTTCTGGCTTAGGTCACCGAGAGTCTCGCTCAGGACAGTCCTCTGGCTAC GGTCAACATGGATCTAGCTCAGGTCATTCCTCTACCCATGGGCAACACGGTTCT ACATCAGGACAGTCATCGAGCTGTGGCCAACATGGAGCTAGCTCAGGTCAGTCT TCCAGCCACGGTCAGCATGGCTCTGGCTCAAGTCAGTCTTCTGGCTATGGCCGA CAGGGCTCTGGATCTGGCCAGTCTCCAGGCCACGGCCAGCGTGGGTCTGGGTCA AGGCAGTCTCCCAGCTACGGCCGACATGGGTCTGGCTCCGGTCGGTCTTCCAGC AGTGGCCAACATGGGTCTGGCTTAGGCGAGTCTTCTGGCTTTGGTCACCACGAG TCTAGCTCAGGGCAGTCCTCTAGTTACAGTCAGCATGGGTCTGGCTCAGGTCAC TCCTCTGGCTACGGACAACACGGCTCTAGATCAGGACAGTCATCTAGGGGTGAA CGACACGGATCTAGCTCAGGTTCGTCTTCCCACTATGGTCAGCATGGGTCTGGC TCCCGTCAGTCTTCGGGCCACGGCCGACAAGGGTCTGGATCTGGCCATTCCCCT AGCCGCGGCCGACATGGGTCCGGTTTGGGGCACTCCTCCAGCCACGGCCAACAT GGGTCTGGCTCAGGTCGTTCTTCCAGCCGTGGCCCATATGAGTCTCGCTCGGGT CACTCTTCTGTCTTTGGTCAACATGAGTCTGGCTCAGGACATTCCTCTGCTTAC AGTCAGCATGGTAGTGGCTCAGGGCACTTCTGTAGCCAAGGACAGCATGGTTCT ACATCAGGACAGTCATCAACCTTTGACCAGGAGGGATCTAGCACAGGCCAGTCT 
TCCAGCTATGGCCACCGTGGCTCTGGCTCCAGTCAGTCTTCTGGCTATGGCCGA CATGGGGCTGGATCTGGCCAGTCTCCTAGTCGCGGCCGACATGGGTCCGGTTCT GGGCACTCTTCCAGCTACGGCCAACATGGGTCTGGCTCCGGTTGGTCTTCCAGC AGTGGCCGACATGGGTCTGGCTCAGGTCAGTCTTCTGGATTTGGTCACCACGAG TCTAGCTCATGGCAGTCCTCTGGTTGCACTCAGCATGGATCTGGCTCAGGTCAC TCCTCCAGCTACGAACAACACGGCTCTAGGTCAGGACAGTCATCTAGGGGTGAA CGACACGGATCTAGCTCAGGTTCATCTTCCAGCTATGGTCAGCATGGGTCTGGC TCCCGTCAGTCTTTGGGCCACGGCCAACATGGGTCTGGATCTGGCCAGTCTCCT AGCCCTAGCCGTGGCCGACATGGGTCTGGTTCTGGGCAGTCTTCCAGCTACAGC CCATATGGGTCTGGCTCAGGGTGGTCTTCCAGCCGTGGCCCATATGAGTCTGGC TCCAGTCACTCTTCTGGCTTAGGTCACCGAGAGTCTCGCTCAGGACAGTCCTCT GGCTACGGTCAACATGGATCTAGCTCAGGTCATTCCTCTACCCATGGGCAACAT GGTTCTACATCAGGACAGTCATCGAGCTGTGGCCAACATGGAGCTAGCTCAGGT CAGTCTTCCAGCCACGGTCAGCATGGCTCTGGCTCAAGTCAGTCTTCTGGCTAT GGCCGACAGGGCTCTGGATCTGGCCAGTCTCCAGGCCACGGCCAGCGTGGGTCT GGGTCAAGGCAGTCTCCCAGCTACGGCCGACATGGGTCTGGCTCCGGTCGGTCT TCCAGCAGTGGCCAACATGGGTCTGGCTTAGGCGAGTCTTCTGGCTTTGGTCAC CACGAGTCTAGCTCAGGGCAGTCCTCTAGTTACAGTCAGCATGGGTCTGGCTCA GGTCACTCCTCTGGCTACGGACAACACGGCTCTAGATCAGGACAGTCATCTAGG GGTGAACGACACGGATCTAGCTCACGTTCGTCTTCCCGCTATGGTCAGCATGGG TCTGGCTCCCGTCAGTCTTCGGGCCACGGCCGACAAGGGTCTGGATCTGGCCAG TCCCCTAGCCGCGGCCGACATGGGTCCGGTTTGGGGCACTCCTCCAGCCACGGC CAACATGGGTCTGGCTCAGGTCGTTCTTCCAGCCGTGGCCCATATGAGTCTCGC TCGGGTCACTCTTCTGTCTTTGGTCAACATGAGTCTGGCTCAGGACATTCCTCT GCTTACAGTCAGCATGGTAGTGGCTCAGGGCACTTCTGTAGCCAAGGACAGCAT GGTTCTACATCAGGACAGTCATCAACCTTTGACCAGGAGGGATCTAGCACAGGT CAGTCTTCCAGCCACGGTCAGCATGGCTCTGGCTCAAGTCAGTCTTCTAGCTAT GGCCAACAGGGCTCTGGATCTGGCCAGTCTCCTAGTCGCGGCCGACATGGGTCC GGTTCCGGGCACTCTTCCAGCTACGGCCAACATGGGTCTGGCTCCGGTTGGTCT TCCAGCAGTGGCCGACATGGGTCTGGCTCAGGTCAGTCTTCTGGATTTGGTCAC CATGAGTCTAGCTCATGGCAGTCCTCTGGTTACACTCAGCATGGATCTGGCTCA GGTCACTCCTCCAGCTACGAACAACACGGCTCTAGGTCAGGACAGTCATCTAGG GGTGAACAACACGGATCTAGCTCAGGTTCATCTTCCAGCTATGGTCAGCATGGG TCTGGCTCCCGTCAGTCTTTGGGCCACGGCCAACATGGGTCTGGATCTGGCCAG TCTCCTAGCCCTAGCCGTGGCCGACATGGGTCTGGTTCTGGGCAGTCTTCCAGC TACGGCCCATATGGGTCTGGCTCAGGGTGGTCTTCCAGCCGTGGCCCATATGAG TCTGGCTCCGGTCACTCTTCTGGCTTAGGTCACCGAGAGTCTCGCTCAGGACAG TCCTCTGGCTACGGTCAACATGGATCTAGCTCAGGTCATTCCTCTACCCATGGG CAACATGGTTCTGCATCAGGACAGTCATCGAGCTGTGGCCAACATGGAGCTAGC TCAGGTCAGTCTTCCAGCCACGGTCAGCATGGCTCTGGCTCAAGTCAGTCTTCT GGCTATGGCCGACAGGGCTCTGGATCTGGCCAGTCTCCAGGCCACGGCCAGCGT GGGTCTGGGTCAAGGCAGTCTCCCAGCTATGGCCGACATGGGTCTGGCTCCGGT CGGTCTTCCAGCAGTGGCCAACATGGGCCTGGCTTAGGCGAGTCTTCTGGCTTT GGTCACCACGAGTCTAGCTCAGGGCAGTCCTCTAGTTACAGTCAGCATGGGTCT GGCTCAGGTCACTCCTCTGGCTACGGACAACACGGCTCTAGATCAGGACAGTCA TCTAGGGGTGAACGACACGGATCTAGCTCAGGTTCGTCTTCCCGCTATGGTCAG 
CATGGGTCTGGCTCCCGTCAGTCTTCGGGCCACGGCCGACAAGGGTCTGGATCT GGCCATTCCCCTAGCCGCGGCCGACATGGGTCCGGTTCGGGGCACTCCTCCAGC CACGGCCAACATGGGTCTGGCTCAGGTCGTTCTTCCAGCCGTGGCCCATATGAG TCTCGCTCGGGTCACTCTTCTGTCTTTGGTCAACATGAGTCTGGCTCAGGACAT TCCTCTGCTTACAGTCAGCATGGTAGTGGCTCAGGGCACTTCTGTAGCCAAGGA CAGCATGGTTCTACATCAGGACAGTCATCAACCTTTGACCAGGAGGGATCTAGC ACAGGTCAGTCTTCCAGCCACGGTCAGCATGGCTCTGGCTCAAGTCAGTCTTCT AGCTATGGCCAACAGGGCTCTGGATCTGGCCAGTCTCCTAGTCGCGGCCGACAT GGGTCCGGTTCCGGGCACTCTTCCAGCTACGGCCAACATGGGTCTGGCTCCGGT TGGTCTTCCAGCAGTGGCCGACATGGGTCTGGCTCAGGTCAGTCTTCTGGATTT GGTCACCACGAGTCTAGCTCATGGCAGTCCTCTGGTTACACTCAGCATGGATCT GGCTCAGGTCACTCCTCCAGCTACGAACAACACGGCTCTAGGTCAGGACAGTCA TCTAGGGGTGAACGACACGGATCTAGCTCAGGTTCATCTTCCAGCTATGGTCAG CATGGGTCTGGCTCCCGTCAGTCTTTGGGCCACGGCCAACATGGGTCTGGATCT GGCCAGTCTCCTAGCCCTAGCCGTGGCCGACATGGGTCTGGTTCTGGGCAGTCT TCCAGCTACAGCCCATATGGGTCTGGCTCAGGGTGGTCTTCCAGCCGTGGCCCA TATGAGTCTGGCTCCGGTCACTCTTCTGGCTTAGGTCACCGAGAGTCTCGCTCA GGACAGTCCTCTGGCTACGGTCAACATGGATCTAGCTCAGGTCATTCCTCTACC CATGGGCAACATGGTTCTACATCAGGACAGTCATCGAGCTGTGGCCAACATGGA GCTAGCTCAGGTCAGTCTTCCAGCCACGGTCAGCATGGCTCTGGCTCAAGTCAG TCTTCTGGCTATGGCCGACAGGGCTCTGGATCTGGCCAGTCTCCAGGCCACGGC CAGCGTGGGTCTGGGTCAAGGCAGTCTCCCAGCTACGGCCGACATGGGTCTGGC TCCGGTCGGTCTTCCAGCAGTGGCCAACATGGGTCTGGCTTAGGCGAGTCTTCT GGCTTTGGTCACCACGAGTCTAGCTCAGGGCAGTCCTCTAGTTACAGTCAGCAT GGGTCTGGCTCAGGTCACTCCTCTGGCTACGGACAACACGGCTCTAGATCAGGA CAGTCATCTAGGGGTGAACGACACGGATCTAGCTCAGGTTCGTCTTCCCACTAT GGTCAGCATGGGTCTGGCTCCCGTCAGTCTTCGGGCCACGGCCGACAAGGGTCT GGATCTGGCCAGTCCCCTAGCCGCGGCCGACATGGGTCCGGTTTGGGGCACTCC TCCAGCCACGGCCAACATGGGTCTGGCTCAGGTCGTTCTTCCAGCCGTGGCCCA TATGAGTCTCGCTTGGGTCACTCTTCTGTCTTTGGTCAACATGAGTCTGGCTCA GGACATTCCTCTGCTTACAGTCAGCATGGTAGTGGCTCAGGGCACTTCTGTAGC CAAGGACAGCATGGTTCTACATCAGGACAGTCATCAACCTTTGACCAGGAGGGA TCTAGCACAGGCCAGTCTTCCAGCTATGGCCACCGTGGCTCTGGCTCCAGTCAG TCTTCTGGCTATGGCCGACATGGGGCTGGATCTGGCCAGTCTCTTAGCCACGGC CGACACGGGTCTGGTTCAGGGCAGTCTTCCAGCTACGGCCAACATGGGTCTGGC TCAGGACAGTCCTCTGGTTATAGTCAGCATGGAAGTGGCTCAGGGCAAGATGGG TATTCTTATTGCAAAGGAGGAAGTAACCATGATGGGGGAAGTTCTGGCTCATAT TTTCTCAGTTTTCCTAGTAGCACTTCACCCTATGAATATGTCCAAGAGCAGAGG TGCTACTTTTATCAG

SmaI

CCCGGG

ECORI

GAATTC 


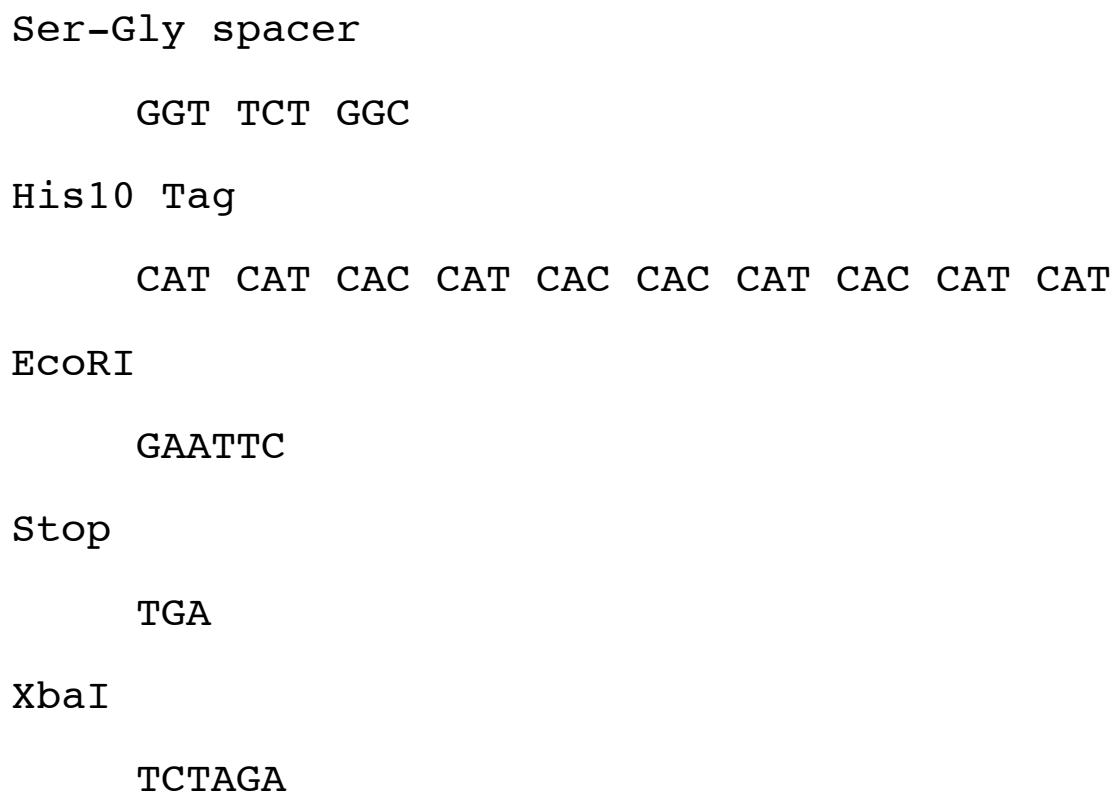

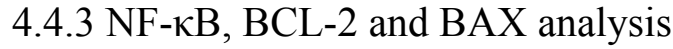

Immediately after transfection, cells were plated on $12 \mathrm{~mm}, 0.1 \%$ gelatin-coated round glass coverslips in 24-well plates and cultured for 16 hours before immunofluorescence. Cells were washed, fixed for 15 minutes in 4\% PFA, then permeabilized in icecold methanol for 1 minute. Cells were then blocked with blocking buffer for 1 hour and

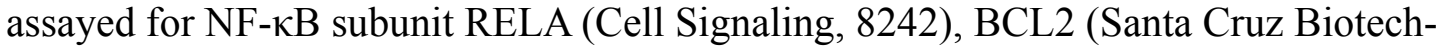
nologies, sc-492), or BAX (Santa Cruz Biotechnologies, sc-493) at 1:100 dilution in blocking buffer for 1 hour. After washing, cells were incubated with 1:200 diluted in blocking buffer rabbit-anti goat AlexaFluor 594 secondary antibody (Invitrogen, A11012). If mitochondria staining was required, cells were incubated with 100nM MitoTracker Deep Red (Invitrogen, M22426) for 15 minutes prior to initiation of the staining 
protocol (before fixation). NF-אB and BCL2 images were analyzed with FIJI (NIH).

BCL2 colocalization data were measured via the Coloc2 toolbox.

\subsubsection{Hydrogen peroxide and JSH-23 treatment}

If required, cells were pretreated with $25 \mu \mathrm{M} \mathrm{JSH}-23$ (Sigma, J4455) in DMSO for 12 hours (initiated 4 hours post-transfection). In experiments requiring hydrogen peroxide treatment, 16 hours post-transfection, cells were incubated with $600 \mu \mathrm{M}$ hydrogen peroxide (Sigma, 323381) in PBS for 4 hours in normal culture conditions. Where JSH23 and $\mathrm{H}_{2} \mathrm{O}_{2}$ were used in combination, JSH-23 was not removed while incubating with $\mathrm{H}_{2} \mathrm{O}_{2}$.

\subsubsection{TUNEL analysis}

For TUNEL analysis, the Click-iT TUNEL Alexa Fluor 594 kit was purchased from Invitrogen (C10246) and used per manufacturer's instructions. For paraffin tissue sections, the TUNEL kit was used after deparaffinization and antigen retrieval. After TUNEL staining, sections were stained with CD34 using previously described protocols. For use with EGFP transfected cells, a specially-formulated Click-iT reaction buffer was kindly provided by Invitrogen Technical Support to eliminated EGFP quenching. Upon completion of the TUNEL assay, cells were counterstained with DAPI, mounted and images were taken using an Olympus BX-41 microscope and QImaging Retiga-2000R cam- 
era microscope (20X objective). TUNEL-positive, EGFP-positive cells were counted and divided by the number of EGFP-positive cells to form a "percent TUNEL cells" metric. 
CHAPTER 5: FUTURE DIRECTIONS AND IMPACT 


\section{$\underline{5.1 \text { Future Directions }}$}

The work presented in Chapters 2, 3 and 4 describes the discovery, validation, functional and mechanistic action of hornerin. However, as with all discoveries, there are several exciting avenues that should be explored further to more completely understand hornerin and its potential as a therapeutic target. Potential and necessary further investigations include confirming that hornerin follows suit with the rest of the S100 family as a calcium binding protein, identifying the mechanism behind hornerin induced NF- $\kappa \mathrm{B}$ activation, and exploring the function of hornerin in tumor cells.

\subsubsection{Confirmation of hornerin as a calcium binding protein}

It is important to understand the molecular mechanism of hornerin signaling in tumor vasculature and endothelial cells in vitro to aid the development a novel therapeutic strategies. Hornerin is a 282 kilodalton protein with 2 predicted EF-hand calcium binding domains at the N-terminal followed by 6 tandem repeats containing mostly glycine and serine residues (135) (Figure 5.1A). As mentioned earlier, hornerin is in the S100 family, a family of low molecular weight proteins primarily consisting of 2 EFhands and calcium binding domains. S100 proteins are involved in many cellular activities including protein phosphorylation and signaling, calcium homeostasis, cytoskeleton dynamics, cell growth and differentiation. We have shown that hornerin is involved in endothelial cell survival through NF- $\mathrm{BB}$ activation. Activation could require calcium binding as an initial first step. Most S100 proteins bind calcium in the EF-hands. S100 
proteins dimerize (183) with or without calcium and sometimes form tetramers from 2 S100 dimers (184). Homodimers are most common, but in some cases heterodimers form. Once dimerized, S100 signaling is activated by calcium induced conformational changes that expose hydrophobic regions $(185,186)$. These hydrophobic regions interact with target proteins such as p53 (186). As shown in Figure 5.1B, hornerin shares great sequence homology with other S100 family members, in EF-hand sequence, calcium binding domains and dimerization domains (187). Although hornerin is much larger than most S100 proteins, it could still potentially bind calcium, as a similar-sized S100 family member profilaggrin readily binds calcium (188). Therefore, we predict that hornerin will indeed bind calcium in both EF-hands and this binding enables downstream signaling important in endothelial cell survival.

Both live cell (in vivo) and solution studies (in vitro) could be utilized to confirm that hornerin binds calcium. Calcium binding studies have traditionally been completed via aromatic residue spectroscopy (189) or calcium indicator dye studies (190). Live cell imaging has the potential to provide great information, but there are many intracellular calcium binding proteins that naturally exist. Hornerin calcium binding would therefore be difficult to isolate and observe above the noise from other calcium binding proteins. Therefore, the most easily controlled experiments involve studying calcium binding of isolated or recombinantly expressed protein in solution. Recombinant expression offers a virtually limitless supply of protein as expressions can be scaled to suit investigator needs. As shown in Figure 4.1A, 2 affinity purification tags (FLAG and His 10 ) were included on the hornerin full-length construct to aid in isolation of recombinantly produced 


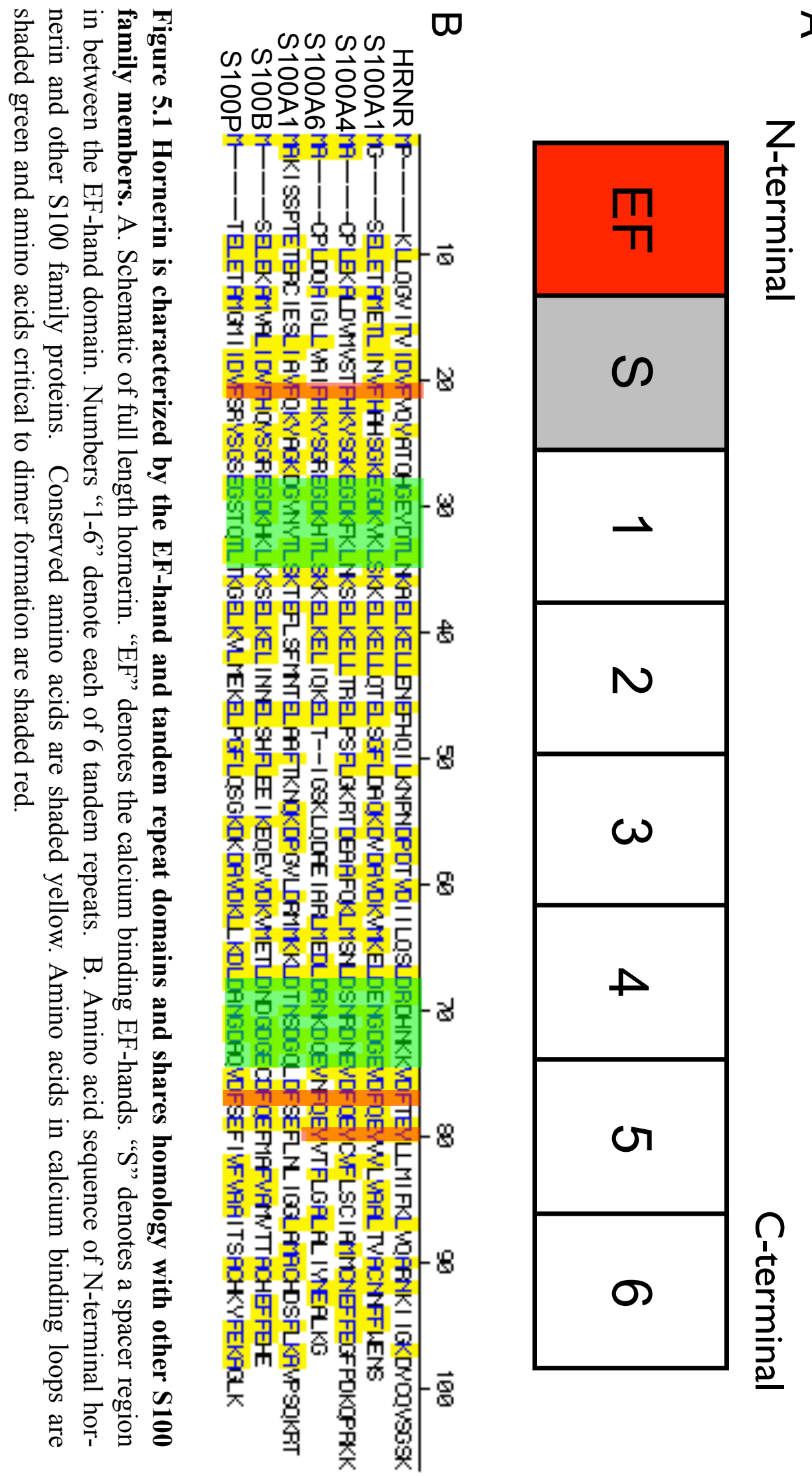


protein. Further, recombinant proteins can easily be mutated to interfere with predicted sites of calcium binding, essentially creating a negative control.

Preliminary efforts to express recombinant hornerin in HEK-293 cells confirm that our recombinant hornerin is present in whole cell lysates (data not shown). However, nickel purification failed to yield purified recombinant hornerin detected by coomassie staining or anti-his immunoblotting. Optimization of recombinant hornerin production and purification must be completed before calcium binding studies can be completed. Work is currently underway to optimize expression and purification so that these studies may be completed. If these studies confirm that hornerin is indeed a calcium binding protein, a potential point of therapeutic intervention could become viable.

\subsubsection{Identification of hornerin induced NF-kB activation mechanism}

As we have shown in Chapter 4, hornerin expression induced NF-kB activation and subsequent translocation into the nucleus. Translocated NF- $\mathrm{\kappa B}$ resulted in BCL2 localization to the mitochondria, where BCL2 exerts its anti-apoptotic action. This sequence of events is hypothesized to contribute to protection from oxidative stress induced cell death in hornerin expressing HUVECs. Knowledge of the mechanism of NF- $\mathrm{kB}$ activation could be exploited as a possible therapeutic intervention. Preventing NF- $\mathrm{KB}$ activation could sensitize cells to oxidative stress that is ever-present in the tumor milieu. In fact, constitutive NF- $\mathrm{KB}$ activation leads to chemo (191) and radioresistance (192) in some cancers and blockade of NF- $\mathrm{kB}$ translocation has been known to sensitize cells to chemotherapeutics (193). 


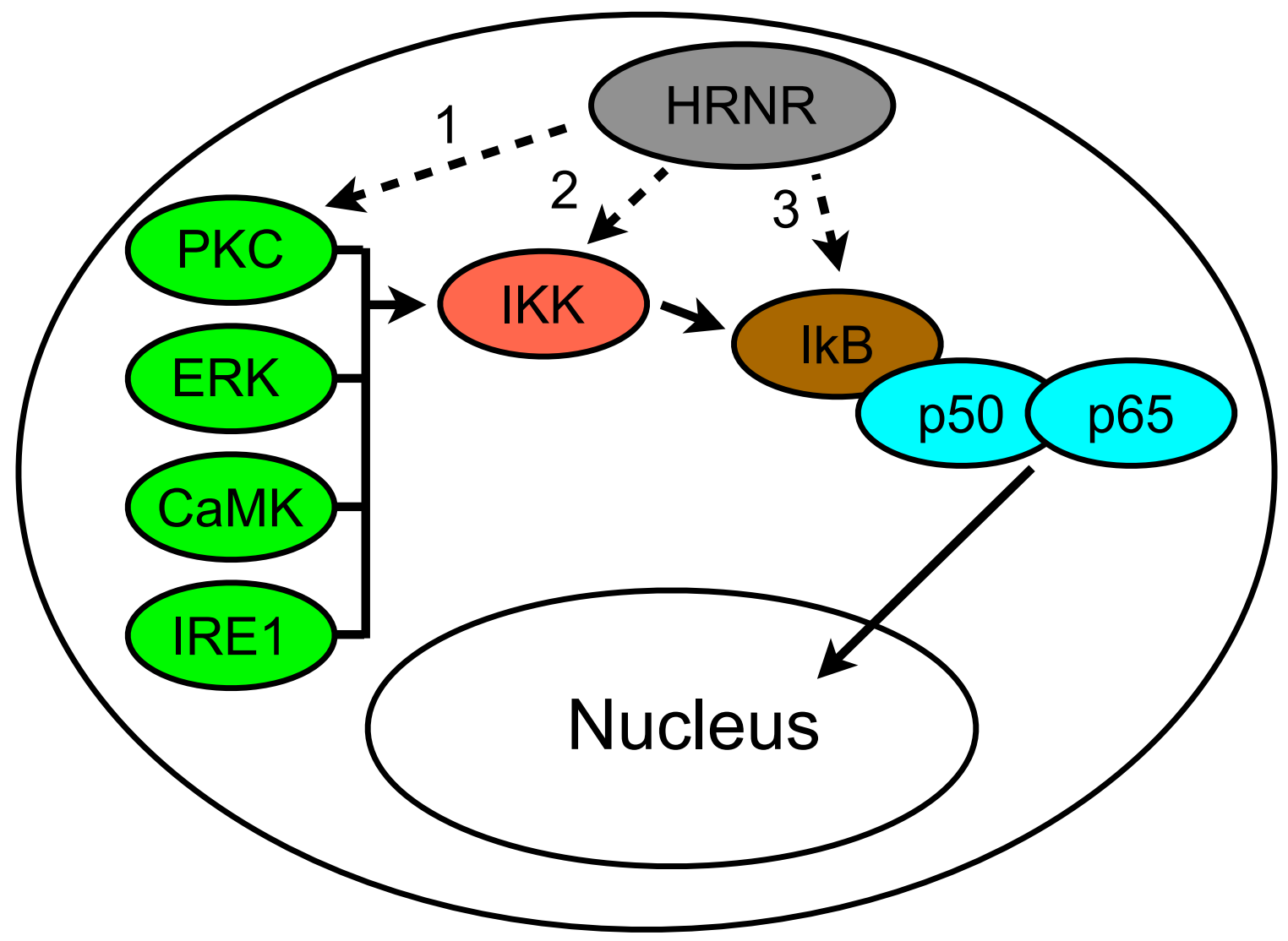

Figure 5.2 Hornerin could potentially act at several points in the NF- $x$ B activation cascade. The cannonical NF- $\kappa \mathrm{B}$ activation cascade is shown via solid line arrows. Typically, some stressor or signaling protein (PKC, ERK, CaMK, or IRE1) activates IKK, which then phosphorylates the IkB-NF- $x \mathrm{~B}$ dimer (composed of p50 and p65, in this case). This results in ubiquitination and degradation of $\mathrm{IkB}$, release and nuclear translocation of NF-xB. The dashed line arrows and numbers represent 3 Paths where hornerin could potentially increase NF- $x \mathrm{~B}$ translocation as observed in Chapter 4. 
The NF-kB activation/translocation pathway is one of the most studied and wellcharacterized pathways. The NF- $\mathrm{BB}$ family is composed of transcription factors that exist as homo or heterodimers of 5 subunits, RelA (p65), c-Rel, RelB, p50 (NF-kB1) and p52 (NF-kB2) (193). In unstimulated cells, NF- $\mathrm{KB}$ dimers are sequestered in the cytoplasm by binding of inhibitory proteins called IkBs. Phosphorylation of IкB by IкBkinase (IKK) results in ubiquitination of IкB and eventual degradation. Subsequently, $\mathrm{NF}-\mathrm{\kappa B}$ is no longer sequestered in the cytoplasm and translocates to the nucleus where it induces transduction of target genes (193).

The linearity of NF- $\mathrm{KB}$ activation allows the formation of narrowed hypotheses. Hornerin most likely 1) directly or indirectly increases IKK activity or 2) phosphorylates I $\kappa \mathrm{B}$ to induce NF- $\mathrm{KB}$ activation and translocation. IKK activation is not well characterized, but it is known to be the result of phosphorylation of an activation loop (194). Several signaling proteins have been implicated in IKK activation, including PKC, ERK, CaMK, and IRE1 $(195,196)$. Perhaps hornerin directly or indirectly stimulates one of these IKK activating proteins, thereby initiating the NF- $\mathrm{kB}$ activation cascade. Alternatively, hornerin could stimulate other signaling proteins that phosphorylate IкBs (not IKKs), thereby releasing NF- $\mathrm{kB}$ from its cytoplasmic, sequestered location. A summary of proposed sites of hornerin interaction is shown in Figure 5.2.

To elucidate the mechanism of hornerin induced NF- $\mathrm{kB}$ activation, a top-down approach is best. The NF-kB translocation inhibitor used in Chapter 4, JSH-23, does not

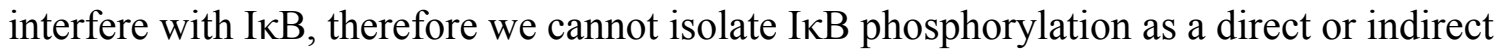
signaling target of hornerin. Mechanistic studies like these are best completed via hor- 
A
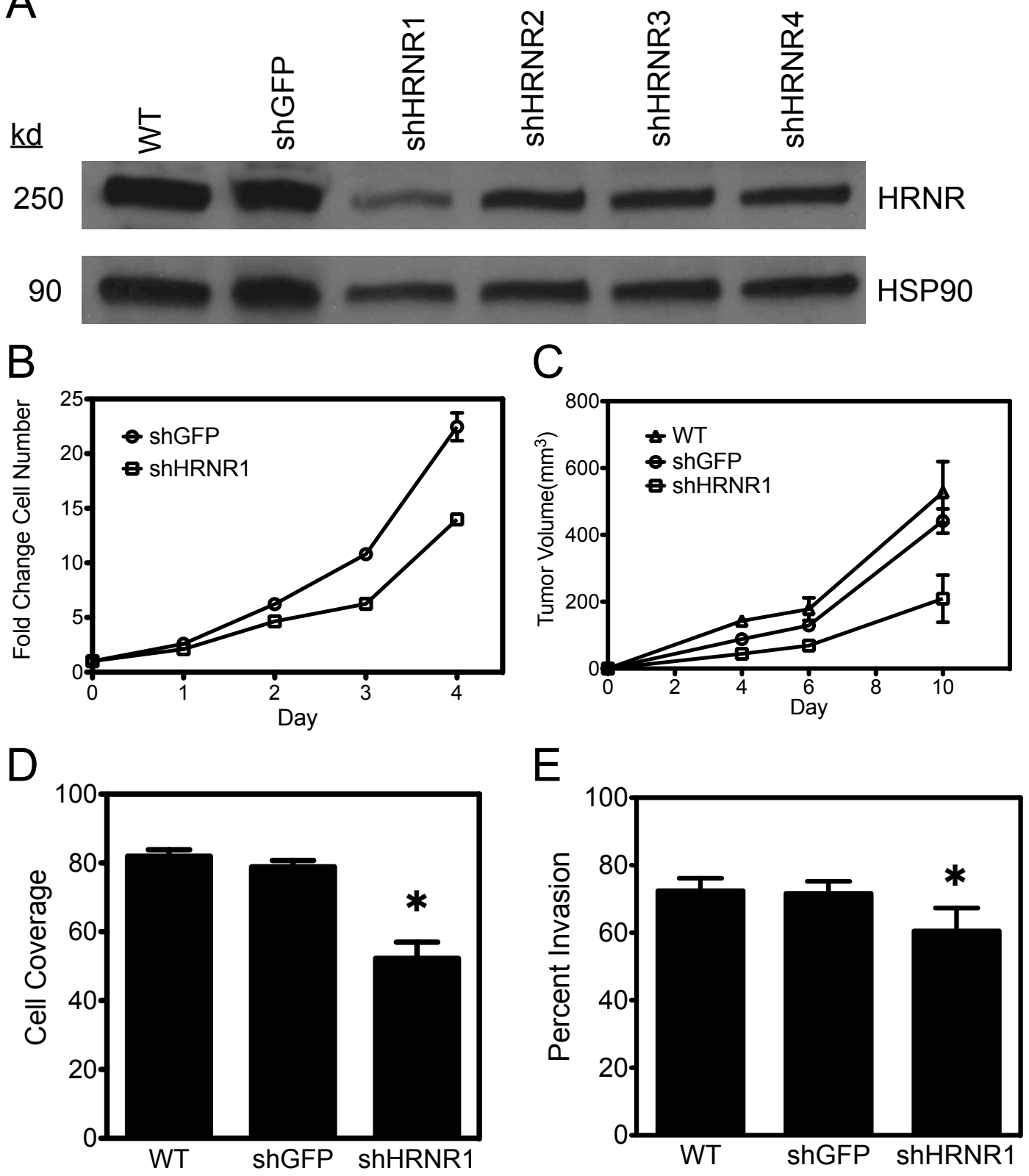

Figure 5.3 Hornerin knockdown reduces L3.6pl cell growth and migration. A. Western blot of hornerin knockdown cell lysates. HSP90 is shown as a loading control. B. Plot of fold change in cell number (luciferase signal) over Day 0 (time of plating). $\mathrm{n}=5$ per group, each day. C. In vivo tumor growth curve. $n=4$ tumors per group. D. L36.pl migration as measured by the percent cell coverage in a $20 \mathrm{X}$ imaging field. $n=12$ images per cell type (4 images per experiment, 3 replicate experiments). E. L3.6pl tumor cell invasion. * $\mathrm{p}<0.05$, one-tailed, unpaired student's t-test compared to WT and shGFP controls. All data represented as mean \pm s.e.m. 
nerin transfection into cells harboring stable knockdowns of IKK or IKK activators or an

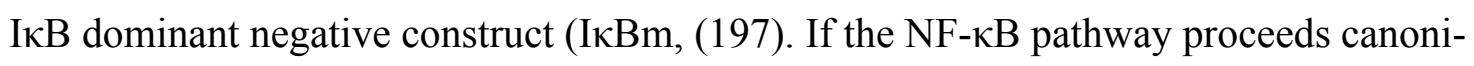
cally, hornerin transfection into an IkB dominant-negative cell line should reduce NF-kB translocation. To investigate whether the effect is mediated at the IKK level (Path 1 and 2 in Figure 5.2), NF- $\mathrm{kB}$ translocation in hornerin transfected sh $I K K$ cells should be studied. If hornerin contributes at the IKK level, reduced NF- $\mathrm{BB}$ translocation should be observed in these experiments. If NF- $\mathrm{kB}$ translocation is unchanged, then hornerin likely activates IкB directly or indirectly (Path 3). Further, if knockdown of any of the known IKK activating proteins reduces NF- $\mathrm{BB}$ activation, then hornerin induced NF- $\mathrm{BB}$ activation likely proceeds through that knocked down protein (Path 1, Figure 5.2). However, if knockdown of IKK activators fail to reduce NF- $\mathrm{KB}$ activation, another activating protein or hornerin-IKK activation (Path 2) mechanisms are likely present. Understanding this cascade is central to identifying possible points of therapeutic intervention that could sensitize cells to oxidative stress.

\subsubsection{Investigation into the role of hornerin in tumor progression}

As shown in Chapter 2, hornerin expression is not limited to endothelial cells. Resected PDAC tumor sections have hornerin expression in both tumor and endothelial cells (Figure 2.8). Additionally, a human tumor microarray revealed that hornerin is expressed in a variety of tumors including pancreatic, renal, breast, and colon (Figure 2.9). Hornerin involvement in tumor cell progression would be a significant finding that could motivate the efforts to target endothelial and tumor cell hornerin to increase therapeutic 
efficacy. In the knockdown studies in Chapter 3, endothelial hornerin was specifically targeted based on the selection of tumor model. Thus, we can not comment on the role of hornerin in tumor progression based on these experiments.

In vitro, tumor cells often have increased proliferation, migration and invasion each are included in Hanahan and Weinberg's "Hallmarks of Cancer" $(5,6)$. Unlike HUVECs where hornerin expression is absent in non-stimulated cells, hornerin is expressed in tumor cells. To investigate the role of hornerin in proliferation, migration and invasion, we reduced hornerin expression through the generation of stable hornerin knockdown cells. As shown in Figure 5.3A, hornerin expression was greatly reduced in the shHRNR1 L3.6pl knocked down cell line, but not in wild-type (WT), shGFP (transfection control), shHRNR2, shHRNR3, or shHRNR4. Therefore, subsequent knockdown studies were completed using the shHRNR1 hornerin knockdown cell line and WT and shGFP cell lines as controls. First, tumor cell proliferation was investigated. Hornerin knockdown resulted in decreased proliferation, as shHRNR1 cell number was reduced by $38 \%$ compared to shGFP control cells (Figure 5.3B). These experiments were extended in vivo as WT, shGFP and shHRNR1 L3.6pl tumor cells were implanted on the flanks of mice and tumor volume was monitored. As shown in Figure 5.3C, shHRNR1 tumor volume was reduced $60 \%$ and $53 \%$ compared to WT and shGFP tumors, respectively. These data confirm that hornerin expression contributes to increased tumor progression and may therefore be a tumor cell therapeutic target.

Next, we investigated the effect of hornerin knockdown on migration and invasion. As shown in Figure 5.3D, hornerin knockdown lead to significant reductions in mi- 
gration (35\%) compared to WT and shGFP cells. Knockdown of hornerin resulted in a $15 \%$ reduction in invasion. Reductions in tumor growth and migration resulting from hornerin knockdown establish that hornerin expression is important in tumor progression.

To understand the role of hornerin in tumor progression, there are several routes to pursue first. Since NF-kB is known to act as a transcription factor that promotes cell survival and migration (198), determining whether NF- $\mathrm{kB}$ translocation is induced by hornerin expression in tumor cells could explain our preliminary findings. If hornerin knockdown cells result in less translocated NF-kB, then hornerin may promote tumor progression though NF-kB activation, as we previously observed in endothelial cells. S100 proteins are important in a variety of cellular processes including phosphorylation and signaling, cytoskeletal dynamics, cell growth and differentiation (199). Understanding how these S100 proteins signal and exert their cellular effects could lead to understanding the role of hornerin in tumor progression. The data presented in this dissertation implicate hornerin as an important mediator of both tumor and tumor vessel development; therefore, determining the mechanism of action in both cell types will pave the way for the creation of novel targeted strategies to improve cancer patient care and overall survival.

\subsection{Materials and Methods}

5.2.1 Hornerin sequence homology 
Hornerin and selected S100 protein amino acid sequences were obtained from the NCBI Protein Database. Sequences were aligned and homology was displayed using the Lasergene MegAlign software package from DNASTAR.

\subsubsection{Stable knockdown cell production}

To produce stable knockdown cell lines through lentiviral transfection, lentivirus was produced via human pLKO.1 lentiviral shRNA target gene set (Thermo Scientific) specific for hornerin. Briefly, 293T cells were incubated with vendor supplied pLKO.1 vector, psPAX2 gag-pol packaging vector, and pMDG.2 VSV-G envelope vector, using Lipofectamine LTX (Life Technologies) as a transfection aid. After 24 hours, viruscontaining supernatant was collected and used fresh or stored at $-80^{\circ} \mathrm{C}$. Next, virus particles were incubated with L3.6pl cells in a 6-well plate. 48 hours later, cells were transferred to a larger T-75 flask and incubated with $3 \mu \mathrm{g} / \mathrm{mL}$ puromycin for antibiotic selection. Cells were grown in normal culture conditions in normal media with puromycin supplement for 2 weeks to obtain stable knockdown cells.

\subsubsection{Cell proliferation assay}

For cell proliferation assays, the Cell-titer Glo Luminescent Cell Viability Assay (Promega, G7571) was used. Cells were grown in white Corning 96-well plates for luminescence imaging. Cell viability was measured per manufacturer's instructions. Lumi- 
nescent signal measured by FLUOStar OPTIMA (Life Technologies) equipped with luminescent filter set.

\subsubsection{Tumor growth curve analysis}

$5 \times 10^{5}$ cells were implanted in the flanks of athymic nude mice. Tumors were monitored semi-weekly and tumor dimensions were measured by caliper. Tumor volume was calculated using the formula (width) $)^{2} /$ length, where width represents the minor axis and length represents the major axis.

\subsubsection{Migration and invasion assay}

For migration assays, 24-well, 8 micron migration transwell inserts were used. For invasion assays, 24-well, 8 micron matrigel-coated transwell inserts were used. Briefly, $2 \times 10^{4}$ cells in serum-free media were plated on freshly rehydrated membranes. The well below the transwell insert was filled with normal growth media. After overnight incubation in normal culture conditions, non-migrating cells were removed with cotton swab. Membranes were washed, then cells were fixed in 4\% PFA before being stained by $1 \%$ crystal violet in $\mathrm{ddH}_{2} \mathrm{O}$. Crystal violet stained migrating cells were imaged by $20 \mathrm{X}$ objective. Migration was measured as percent image frame occupied by crystal violet stain. Invasion percentage was calculated as percent invading cell coverage / average migration coverage for each cell type. 


\section{$\underline{5.3 \text { Impact }}$}

As the work presented in this dissertation was initiated, anti-tumor vessel therapies were emerging as a viable and exciting new therapy - especially those that target the VEGF pathway. Bevicizumab, a humanized monoclonal antibody that acts as a VEGFtrap, was first approved for metastatic colorectal cancer in 2004. That approval had a cascading effect, as bevicizumab was approved for a myriad of cancer types, including glioblastoma, non-small cell lung cancer, and HER-2 negative breast cancer in the following years. Early returns were promising, but substantial gains in patient survival were ultimately absent (62). As a result, optimism for anti-vessel therapies began to wane. We rejected the notion that anti-vessel strategies were not a viable cancer therapy and hypothesized that compensatory pathways may result in tumor recurrence and poor patient survival gains. Therefore, we began an in vivo phage display screen coupled with functional proteomics to find a protein that was important in tumor vessel development, but not mediated by VEGF. In the beginning stages of the search for a non-VEGF mediated tumor vessel protein, our aims were bolstered by the revocation of FDA approval for bevicizumab as a treatment for breast cancer in late 2011 (FDA News Release, November 2011). Independent of our research, Douglas Hanahan, a co-author of one of the most seminal papers in oncology, "The Hallmarks of Cancer" $(5,6)$, was beginning to describe the existence of compensatory pathways in a series of research articles and reviews (81, $82,200)$. The discovery and validation of hornerin as a non-VEGF protein functionally involved in murine and human tumor vessel development, aligns us with the current thought-leaders in anti-tumor vessel therapy. 
The use of phage display based functional proteomics to identify hornerin further bolsters this method as an efficient protein discovery tool. Before functional proteomics methods were created in our laboratory (126), peptides derived from phage screens could only be used for imaging and targeted therapeutic applications. Functional proteomic methods allow the truly unbiased identification of proteins because investigators are not able to unintentionally or intentionally bias the selection of candidate proteins to investigate further based on previous experience or publications. Other genomics and proteomics tools are sometimes susceptible to this type of unintended bias. As a result, our finding of endothelially-expressed hornerin represents the first such finding. Others have described hornerin expression in skin $(135,201-203)$ and breast cancer (204), but our work necessitates further investigation into its role in tumor vessel development. As detailed in the Future Directions section, several potential therapeutic routes are possible based on the work presented in this dissertation. The elucidation of the mechanism of hornerin induced translocation of NF- $\mathrm{kB}$ and whether this can be controlled by calcium binding highlight two points of potential intervention.

Hornerin is not just important in tumor development. With the help of a postdoctoral fellow in the Kelly Laboratory, Siva Dasa, we have preliminarily shown that hornerin is upregulated in the vessels of remodeling cardiac tissue in a reperfusion myocardial infarcation model. Hornerin was also found to be overexpressed in regions of ischemia following myocardial infarction in human patient tissue samples, highlighting the potential clinical significance of hornerin in human cardiac remodeling. Therefore, it seems that hornerin therapeutic interventions should be approached in a context depend- 
ent manner; knockdown is desired in a pathologic situation where vessel growth must be controlled, while expression should be stimulated to encourage physiologic processes such as cardiac vessel remodeling. 
CHAPTER 6: REFERENCES 
1. Department of HHS. National Vital Statistics Report. Vol 6. No. 61.

2. Jemal A, Siegel R, Xu J, Ward E. Cancer statistics, 2010. CA Cancer J Clin. 2010;60:277-300.

3. DeVita VTJ, Chu E. A history of cancer chemotherapy. Cancer Res. 2008;68:8643-53.

4. Cesca M, Bizzaro F, Zucchetti M, Giavazzi R. Tumor Delivery of Chemotherapy Combined with Inhibitors of Angiogenesis and Vascular Targeting Agents. Front Oncol. 2013;3:259.

5. Hanahan D, Weinberg RA. The hallmarks of cancer. Cell. 2000;100:57-70.

6. Hanahan D, Weinberg RA. Hallmarks of cancer: the next generation. Cell. 2011;144:646-74.

7. Ide AG, Baker NH, Warren SL. Vascularization of the Brown Pearce rabbit epithelioma transplant as seen in the transparent ear chamber. Am J Reontgenol. 891-899:891-9.

8. Goodall CM, Feldman R, Sanders AG, Shubik P. Vascular patterns of four transplantable tumors in the hamster (Mesocricetus auratus). Angiology. 1965;16:622-5.

9. FEIGIN I, ALLEN LB, LIPKIN L, GROSS SW. The endothelial hyperplasia of the cerebral blood vessels with brain tumors, and its sarcomatous transformation. Cancer. 1958;11:264-77.

10. Warren BA. The ultrastructure of capillary sprouts induced by melanoma transplants in the golden hamster. J R Microsc Soc. 1966;86:177-87.

11. Folkman J. Tumor angiogenesis: therapeutic implications. N Engl J Med. 1971;285:1182-6.

12. Folkman J. Anti-angiogenesis: new concept for therapy of solid tumors. Ann Surg. 1972;175:409-16.

13. Greene HS. HETEROLOGOUS TRANSPLANTATION OF MAMMALIAN TUMORS : I. THE TRANSFER OF RABBIT TUMORS TO ALIEN SPECIES. J Exp Med. 1941;73:461-74.

14. Folkman J, Merler E, Abernathy C, Williams G. Isolation of a tumor factor responsible for angiogenesis. J Exp Med. 1971;133:275-88.

15. Greenblatt M, Shubi P. Tumor angiogenesis: transfilter diffusion studies in the hamster by the transparent chamber technique. J Natl Cancer Inst. 1968;41:111-24.

16. Bergers G, Benjamin LE. Tumorigenesis and the angiogenic switch. Nat Rev Cancer. 2003;3:401-10.

17. Dvorak HF. Tumors: wounds that do not heal. Similarities between tumor stroma generation and wound healing. N Engl J Med. 1986;315:1650-9.

18. Jain RK. Normalization of tumor vasculature: an emerging concept in antiangiogenic therapy. Science. 2005;307:58-62.

19. Senger DR, Galli SJ, Dvorak AM, Perruzzi CA, Harvey VS, Dvorak HF. Tumor cells secrete a vascular permeability factor that promotes accumulation of ascites fluid. Science. 1983;219:983-5. 
20. Dvorak HF, Brown LF, Detmar M, Dvorak AM. Vascular permeability factor/ vascular endothelial growth factor, microvascular hyperpermeability, and angiogenesis. Am J Pathol. 1995;146:1029-39.

21. Ferrara N, Davis-Smyth T. The biology of vascular endothelial growth factor. Endocr Rev. 1997;18:4-25.

22. de Vries C, Escobedo JA, Ueno H, Houck K, Ferrara N, Williams LT. The fms-like tyrosine kinase, a receptor for vascular endothelial growth factor. Science. 1992;255:989-91.

23. Terman BI, Dougher-Vermazen M, Carrion ME et al. Identification of the KDR tyrosine kinase as a receptor for vascular endothelial cell growth factor. Biochem Biophys Res Commun. 1992;187:1579-86.

24. Pajusola K, Aprelikova O, Korhonen J et al. FLT4 receptor tyrosine kinase contains seven immunoglobulin-like loops and is expressed in multiple human tissues and cell lines. Cancer Res. 1992;52:5738-43.

25. Waltenberger J, Claesson-Welsh L, Siegbahn A, Shibuya M, Heldin CH. Different signal transduction properties of KDR and Flt1, two receptors for vascular endothelial growth factor. J Biol Chem. 1994;269:26988-95.

26. Schwartz JD, Rowinsky EK, Youssoufian H, Pytowski B, Wu Y. Vascular endothelial growth factor receptor-1 in human cancer: concise review and rationale for development of IMC-18F1 (Human antibody targeting vascular endothelial growth factor receptor-1). Cancer. 2010;116:1027-32.

27. Smith NR, Baker D, James NH et al. Vascular endothelial growth factor receptors VEGFR-2 and VEGFR-3 are localized primarily to the vasculature in human primary solid cancers. Clin Cancer Res. 2010;16:3548-61.

28. Roskoski RJ. Vascular endothelial growth factor (VEGF) signaling in tumor progression. Crit Rev Oncol Hematol. 2007;62:179-213.

29. Roberts WG, Palade GE. Increased microvascular permeability and endothelial fenestration induced by vascular endothelial growth factor. J Cell Sci. 1995;108:2369-79.

30. Kevil CG, Payne DK, Mire E, Alexander JS. Vascular permeability factor/vascular endothelial cell growth factor-mediated permeability occurs through disorganization of endothelial junctional proteins. J Biol Chem. 1998;273:15099-103.

31. Connolly DT, Heuvelman DM, Nelson R et al. Tumor vascular permeability factor stimulates endothelial cell growth and angiogenesis. J Clin Invest. 1989;84:1470-8.

32. Morales-Ruiz M, Fulton D, Sowa G et al. Vascular endothelial growth factorstimulated actin reorganization and migration of endothelial cells is regulated via the serine/threonine kinase Akt. Circ Res. 2000;86:892-6.

33. Gerber HP, McMurtrey A, Kowalski J et al. Vascular endothelial growth factor regulates endothelial cell survival through the phosphatidylinositol 3'-kinase/Akt signal transduction pathway. Requirement for Flk-1/KDR activation. J Biol Chem. 1998;273:30336-43. 
34. Valkovic T, Dobrila F, Melato M, Sasso F, Rizzardi C, Jonjic N. Correlation between vascular endothelial growth factor, angiogenesis, and tumor-associated macrophages in invasive ductal breast carcinoma. Virchows Arch. 2002;440:583-8.

35. Wang L, Liu X, Wang H, Wang S. Correlation of the expression of vascular endothelial growth factor and its receptors with microvessel density in ovarian cancer. Oncol Lett. 2013;6:175-80.

36. Carmeliet P, Ferreira V, Breier G et al. Abnormal blood vessel development and lethality in embryos lacking a single VEGF allele. Nature. 1996;380:435-9.

37. Shing Y, Folkman J, Sullivan R, Butterfield C, Murray J, Klagsbrun M. Heparin affinity: purification of a tumor-derived capillary endothelial cell growth factor. Science. 1984;223:1296-9.

38. Nickel W. The unconventional secretory machinery of fibroblast growth factor 2 . Traffic. 2011;12:799-805.

39. Saksela O, Rifkin DB. Release of basic fibroblast growth factor-heparan sulfate complexes from endothelial cells by plasminogen activator-mediated proteolytic activity. J Cell Biol. 1990;110:767-75.

40. Powers CJ, McLeskey SW, Wellstein A. Fibroblast growth factors, their receptors and signaling. Endocr Relat Cancer. 2000;7:165-97.

41. Romo P, Madigan MC, Provis JM, Cullen KM. Differential effects of TGF-beta and FGF-2 on in vitro proliferation and migration of primate retinal endothelial and Muller cells. Acta Ophthalmol. 2011;89:e263-8.

42. Gospodarowicz D, Abraham JA, Schilling J. Isolation and characterization of a vascular endothelial cell mitogen produced by pituitary-derived folliculo stellate cells. Proc Natl Acad Sci U S A. 1989;86:7311-5.

43. Lee JG, Kay EP. FGF-2-induced wound healing in corneal endothelial cells requires Cdc42 activation and Rho inactivation through the phosphatidylinositol 3-kinase pathway. Invest Ophthalmol Vis Sci. 2006;47:1376-86.

44. Montesano R, Vassalli JD, Baird A, Guillemin R, Orci L. Basic fibroblast growth factor induces angiogenesis in vitro. Proc Natl Acad Sci U S A. 1986;83:7297-301.

45. Garcia de la Torre N, Buley I, Wass JA, Jackson DG, Turner HE. Angiogenesis and lymphangiogenesis in parathyroid proliferative lesions. J Clin Endocrinol Metab. 2004;89:2890-6.

46. Zhang W, Chu YQ, Ye ZY, Zhao ZS, Tao HQ. Expression of hepatocyte growth factor and basic fibroblast growth factor as prognostic indicators in gastric cancer. Anat Rec (Hoboken). 2009;292:1114-21.

47. Kao S, Mo J, Baird A, Eliceiri BP. Basic fibroblast growth factor in an animal model of spontaneous mammary tumor progression. Oncol Rep. 2012;27:1807-14.

48. Foletti A, Ackermann J, Schmidt A, Hummler E, Beermann F. Absence of fibroblast growth factor 2 does not prevent tumor formation originating from the RPE. Oncogene. 2002;21:1841-7.

49. Betsholtz C, Karlsson L, Lindahl P. Developmental roles of platelet-derived growth factors. Bioessays. 2001;23:494-507. 
50. Nicosia RF, Nicosia SV, Smith M. Vascular endothelial growth factor, plateletderived growth factor, and insulin-like growth factor-1 promote rat aortic angiogenesis in vitro. Am J Pathol. 1994;145:1023-9.

51. Lindahl P, Johansson BR, Leveen P, Betsholtz C. Pericyte loss and microaneurysm formation in PDGF-B-deficient mice. Science. 1997;277:242-5.

52. Papetti M, Herman IM. Mechanisms of normal and tumor-derived angiogenesis. Am J Physiol Cell Physiol. 2002;282:C947-70.

53. Folkman J. Looking for a good endothelial address. Cancer Cell. 2002;1:113-5.

54. O'Reilly MS, Holmgren L, Shing Y et al. Angiostatin: a novel angiogenesis inhibitor that mediates the suppression of metastases by a Lewis lung carcinoma. Cell. 1994;79:315-28.

55. O'Reilly MS, Holmgren L, Chen C, Folkman J. Angiostatin induces and sustains dormancy of human primary tumors in mice. Nat Med. 1996;2:689-92.

56. O'Reilly MS, Boehm T, Shing Y et al. Endostatin: an endogenous inhibitor of angiogenesis and tumor growth. Cell. 1997;88:277-85.

57. Folkman J. Antiangiogenesis in cancer therapy--endostatin and its mechanisms of action. Exp Cell Res. 2006;312:594-607.

58. Duda DG. Molecular Biomarkers of Response to Antiangiogenic Therapy for Cancer. ISRN Cell Biol. 2012;2012

59. Presta LG, Chen H, O'Connor SJ et al. Humanization of an anti-vascular endothelial growth factor monoclonal antibody for the therapy of solid tumors and other disorders. Cancer Res. 1997;57:4593-9.

60. Gerber HP, Ferrara N. Pharmacology and pharmacodynamics of bevacizumab as monotherapy or in combination with cytotoxic therapy in preclinical studies. Cancer Res. 2005;65:671-80.

61. Hurwitz H, Fehrenbacher L, Novotny W et al. Bevacizumab plus irinotecan, fluorouracil, and leucovorin for metastatic colorectal cancer. N Engl J Med. 2004;350:2335-42.

62. Meadows KL, Hurwitz HI. Anti-VEGF therapies in the clinic. Cold Spring Harb Perspect Med. 2012;2

63. Posey JA, Ng TC, Yang B et al. A phase I study of anti-kinase insert domaincontaining receptor antibody, IMC-1C11, in patients with liver metastases from colorectal carcinoma. Clin Cancer Res. 2003;9:1323-32.

64. Kantarjian H, Sawyers C, Hochhaus A et al. Hematologic and cytogenetic responses to imatinib mesylate in chronic myelogenous leukemia. $\mathrm{N}$ Engl J Med. 2002;346:645-52.

65. Ellis LM, Hicklin DJ. VEGF-targeted therapy: mechanisms of anti-tumour activity. Nat Rev Cancer. 2008;8:579-91.

66. Demetri GD, van Oosterom AT, Garrett CR et al. Efficacy and safety of sunitinib in patients with advanced gastrointestinal stromal tumour after failure of imatinib: a randomised controlled trial. Lancet. 2006;368:1329-38.

67. Motzer RJ, Hutson TE, Tomczak P et al. Sunitinib versus interferon alfa in metastatic renal-cell carcinoma. N Engl J Med. 2007;356:115-24. 
68. Wilhelm SM, Adnane L, Newell P, Villanueva A, Llovet JM, Lynch M. Preclinical overview of sorafenib, a multikinase inhibitor that targets both Raf and VEGF and PDGF receptor tyrosine kinase signaling. Mol Cancer Ther. 2008;7:3129-40.

69. Llovet JM, Ricci S, Mazzaferro V et al. Sorafenib in advanced hepatocellular carcinoma. N Engl J Med. 2008;359:378-90.

70. Escudier B, Eisen T, Stadler WM et al. Sorafenib in advanced clear-cell renal-cell carcinoma. N Engl J Med. 2007;356:125-34.

71. Gupta S, Spiess PE. The prospects of pazopanib in advanced renal cell carcinoma. Ther Adv Urol. 2013;5:223-32.

72. Sternberg CN, Davis ID, Mardiak J et al. Pazopanib in locally advanced or metastatic renal cell carcinoma: results of a randomized phase III trial. J Clin Oncol. 2010;28:1061-8.

73. Motzer RJ, Hutson TE, Cella D et al. Pazopanib versus sunitinib in metastatic renalcell carcinoma. N Engl J Med. 2013;369:722-31.

74. Rebucci M, Michiels C. Molecular aspects of cancer cell resistance to chemotherapy. Biochem Pharmacol. 2013;85:1219-26.

75. Meacham CE, Morrison SJ. Tumour heterogeneity and cancer cell plasticity. Nature. 2013;501:328-37.

76. Schneider BP, Miller KD. Angiogenesis of breast cancer. J Clin Oncol. 2005;23:1782-90.

77. Ugurel S, Rappl G, Tilgen W, Reinhold U. Increased serum concentration of angiogenic factors in malignant melanoma patients correlates with tumor progression and survival. J Clin Oncol. 2001;19:577-83.

78. Sitohy B, Nagy JA, Jaminet SC, Dvorak HF. Tumor-surrogate blood vessel subtypes exhibit differential susceptibility to anti-VEGF therapy. Cancer Res. 2011;71:7021-8.

79. Miller KD, Sweeney CJ, Sledge GWJ. Can tumor angiogenesis be inhibited without resistance? EXS. 2005;95-112.

80. Gottesman MM. Mechanisms of cancer drug resistance. Annu Rev Med. 2002;53:615-27.

81. Casanovas O, Hicklin DJ, Bergers G, Hanahan D. Drug resistance by evasion of antiangiogenic targeting of VEGF signaling in late-stage pancreatic islet tumors. Cancer Cell. 2005;8:299-309.

82. Allen E, Walters IB, Hanahan D. Brivanib, a dual FGF/VEGF inhibitor, is active both first and second line against mouse pancreatic neuroendocrine tumors developing adaptive/evasive resistance to VEGF inhibition. Clin Cancer Res. 2011;17:5299-310.

83. Lambrechts D, Lenz HJ, de Haas S, Carmeliet P, Scherer SJ. Markers of response for the antiangiogenic agent bevacizumab. J Clin Oncol. 2013;31:1219-30.

84. Dowlati A, Gray R, Sandler AB, Schiller JH, Johnson DH. Cell adhesion molecules, vascular endothelial growth factor, and basic fibroblast growth factor in patients with non-small cell lung cancer treated with chemotherapy with or without 
bevacizumab--an Eastern Cooperative Oncology Group Study. Clin Cancer Res. 2008;14:1407-12.

85. Kopetz S, Hoff PM, Morris JS et al. Phase II trial of infusional fluorouracil, irinotecan, and bevacizumab for metastatic colorectal cancer: efficacy and circulating angiogenic biomarkers associated with therapeutic resistance. J Clin Oncol. 2010;28:453-9.

86. Spellman P. A status report on MAGE. Bioinformatics. 2005;21:3459-60.

87. Brazma A, Hingamp P, Quackenbush J et al. Minimum information about a microarray experiment (MIAME)-toward standards for microarray data. Nat Genet. 2001;29:365-71.

88. Nielsen CB, Cantor M, Dubchak I, Gordon D, Wang T. Visualizing genomes: techniques and challenges. Nat Methods. 2010;7:S5-S15.

89. Lander ES, Linton LM, Birren B et al. Initial sequencing and analysis of the human genome. Nature. 2001;409:860-921.

90. Chin L, Andersen JN, Futreal PA. Cancer genomics: from discovery science to personalized medicine. Nat Med. 2011;17:297-303.

91. Hjortland GO, Meza-Zepeda LA, Beiske K et al. Genome wide single cell analysis of chemotherapy resistant metastatic cells in a case of gastroesophageal adenocarcinoma. BMC Cancer. 2011;11:455.

92. Obara K, Ghazizadeh M, Shimizu $\mathrm{H}$ et al. Comparative genomic hybridization study of genetic changes associated with vindesine resistance in esophageal carcinoma. Int J Oncol. 2002;20:255-60.

93. Osterberg L, Levan K, Partheen K et al. Potential predictive markers of chemotherapy resistance in stage III ovarian serous carcinomas. BMC Cancer. 2009;9:368.

94. Parsons DW, Jones S, Zhang X et al. An integrated genomic analysis of human glioblastoma multiforme. Science. 2008;321:1807-12.

95. Rohle D, Popovici-Muller J, Palaskas N et al. An inhibitor of mutant IDH1 delays growth and promotes differentiation of glioma cells. Science. 2013;340:626-30.

96. Chin L, Hahn WC, Getz G, Meyerson M. Making sense of cancer genomic data. Genes Dev. 2011;25:534-55.

97. Schulze A, Downward J. Analysis of gene expression by microarrays: cell biologist's gold mine or minefield? J Cell Sci. 2000;113 Pt 23:4151-6.

98. Tsunoda T, Nakamura T, Ishimoto $\mathrm{K}$ et al. Upregulated expression of angiogenesis genes and down regulation of cell cycle genes in human colorectal cancer tissue determined by cDNA macroarray. Anticancer Res. 2001;21:137-43.

99. Morrissey C, True LD, Roudier MP et al. Differential expression of angiogenesis associated genes in prostate cancer bone, liver and lymph node metastases. Clin Exp Metastasis. 2008;25:377-88.

100. Campioni M, Ambrogi V, Pompeo E et al. Identification of genes down-regulated during lung cancer progression: a cDNA array study. J Exp Clin Cancer Res. 2008;27:38. 
101. Ghilardi C, Chiorino G, Dossi R, Nagy Z, Giavazzi R, Bani M. Identification of novel vascular markers through gene expression profiling of tumor-derived endothelium. BMC Genomics. 2008;9:201.

102. Seaman S, Stevens J, Yang MY, Logsdon D, Graff-Cherry C, St Croix B. Genes that distinguish physiological and pathological angiogenesis. Cancer Cell. 2007;11:539-54.

103. Gry M, Rimini R, Stromberg S et al. Correlations between RNA and protein expression profiles in 23 human cell lines. BMC Genomics. 2009;10:365.

104. Issaq HJ, Veenstra TD. Proteomic and metabolomic approaches to biomarker discovery. 2013:xv, 472 pages.

105. Anderson L, Anderson NG. High resolution two-dimensional electrophoresis of human plasma proteins. Proc Natl Acad Sci U S A. 1977;74:5421-5.

106. O'Dwyer D, Ralton LD, O'Shea A, Murray GI. The proteomics of colorectal cancer: identification of a protein signature associated with prognosis. PLoS One. 2011;6:e27718.

107. Peng XC, Gong FM, Zhao YW et al. Comparative proteomic approach identifies PKM2 and cofilin-1 as potential diagnostic, prognostic and therapeutic targets for pulmonary adenocarcinoma. PLoS One. 2011;6:e27309.

108. Veenstra TD. Proteomic applications in cancer detection and discovery. 2013:ix, 308 pages.

109. Unlu M, Morgan ME, Minden JS. Difference gel electrophoresis: a single gel method for detecting changes in protein extracts. Electrophoresis. 1997;18:2071-7.

110. Xiao H, Zhang L, Zhou H, Lee JM, Garon EB, Wong DT. Proteomic analysis of human saliva from lung cancer patients using two-dimensional difference gel electrophoresis and mass spectrometry. Mol Cell Proteomics. 2012;11:M111.012112.

111. Beck M, Schmidt A, Malmstroem J et al. The quantitative proteome of a human cell line. Mol Syst Biol. 2011;7:549.

112. Gutmann DA, Mizohata E, Newstead S et al. A high-throughput method for membrane protein solubility screening: the ultracentrifugation dispersity sedimentation assay. Protein Sci. 2007;16:1422-8.

113. Smith GP. Filamentous fusion phage: novel expression vectors that display cloned antigens on the virion surface. Science. 1985;228:1315-7.

114. Vodnik M, Zager U, Strukelj B, Lunder M. Phage display: selecting straws instead of a needle from a haystack. Molecules. 2011;16:790-817.

115. Bratkovic T. Progress in phage display: evolution of the technique and its application. Cell Mol Life Sci. 2010;67:749-67.

116. Kelly KA, Bardeesy N, Anbazhagan R et al. Targeted nanoparticles for imaging incipient pancreatic ductal adenocarcinoma. PLoS Med. 2008;5:e85.

117. Kelly KA, Waterman P, Weissleder R. In vivo imaging of molecularly targeted phage. Neoplasia. 2006;8:1011-8.

118. Sun Y, Shukla GS, Weaver D, Pero SC, Krag DN. Phage-display selection on tumor histological specimens with laser capture microdissection. J Immunol Methods. 2009;347:46-53. 
119. Kelly KA, Nahrendorf M, Yu AM, Reynolds F, Weissleder R. In vivo phage display selection yields atherosclerotic plaque targeted peptides for imaging. Mol Imaging Biol. 2006;8:201-7.

120. Krag DN, Shukla GS, Shen GP et al. Selection of tumor-binding ligands in cancer patients with phage display libraries. Cancer Res. 2006;66:7724-33.

121. Ruoslahti E. Vascular zip codes in angiogenesis and metastasis. Biochem Soc Trans. 2004;32:397-402.

122. Arap W, Pasqualini R, Ruoslahti E. Cancer treatment by targeted drug delivery to tumor vasculature in a mouse model. Science. 1998;279:377-80.

123. Rajotte D, Arap W, Hagedorn M, Koivunen E, Pasqualini R, Ruoslahti E. Molecular heterogeneity of the vascular endothelium revealed by in vivo phage display. J Clin Invest. 1998;102:430-7.

124. Ruoslahti E, Pierschbacher MD. Arg-Gly-Asp: a versatile cell recognition signal. Cell. 1986;44:517-8.

125. Ruoslahti E. RGD and other recognition sequences for integrins. Annu Rev Cell Dev Biol. 1996;12:697-715.

126. Reynolds F, Panneer N, Tutino CM et al. A functional proteomic method for biomarker discovery. PLoS One. 2011;6:e22471.

127. Folkman J. Angiogenesis in cancer, vascular, rheumatoid and other disease. Nat Med. 1995;1:27-31.

128. Giatromanolaki A, Sivridis E, Simopoulos C et al. Differential assessment of angiogenic activity and of vascular survival ability (VSA) in breast cancer. Clin Exp Metastasis. 2002; 19:673-9.

129. Kindler HL, Ioka T, Richel DJ et al. Axitinib plus gemcitabine versus placebo plus gemcitabine in patients with advanced pancreatic adenocarcinoma: a double-blind randomised phase 3 study. Lancet Oncol. 2011;12:256-62.

130. Lang I, Brodowicz T, Ryvo L et al. Bevacizumab plus paclitaxel versus bevacizumab plus capecitabine as first-line treatment for HER2-negative metastatic breast cancer: interim efficacy results of the randomised, open-label, non-inferiority, phase 3 TURANDOT trial. Lancet Oncol. 2013;14:125-33.

131. Baselga J, Cortes J, Kim SB et al. Pertuzumab plus trastuzumab plus docetaxel for metastatic breast cancer. N Engl J Med. 2012;366:109-19.

132. Kaufman B, Mackey JR, Clemens MR et al. Trastuzumab plus anastrozole versus anastrozole alone for the treatment of postmenopausal women with human epidermal growth factor receptor 2-positive, hormone receptor-positive metastatic breast cancer: results from the randomized phase III TAnDEM study. J Clin Oncol. 2009;27:5529-37.

133. Johnston S, Pippen JJ, Pivot X et al. Lapatinib combined with letrozole versus letrozole and placebo as first-line therapy for postmenopausal hormone receptorpositive metastatic breast cancer. J Clin Oncol. 2009;27:5538-46.

134. Al-Lazikani B, Banerji U, Workman P. Combinatorial drug therapy for cancer in the post-genomic era. Nat Biotechnol. 2012;30:679-92. 
135. Wu Z, Meyer-Hoffert U, Reithmayer K et al. Highly complex peptide aggregates of the S100 fused-type protein hornerin are present in human skin. J Invest Dermatol. 2009;129:1446-58.

136. Igarashi J, Erwin PA, Dantas AP, Chen H, Michel T. VEGF induces S1P1 receptors in endothelial cells: Implications for cross-talk between sphingolipid and growth factor receptors. Proc Natl Acad Sci U S A. 2003;100:10664-9.

137. Carmeliet P. VEGF as a key mediator of angiogenesis in cancer. Oncology. 2005;69 Suppl 3:4-10.

138. Bruns CJ, Harbison MT, Kuniyasu H, Eue I, Fidler IJ. In vivo selection and characterization of metastatic variants from human pancreatic adenocarcinoma by using orthotopic implantation in nude mice. Neoplasia. 1999;1:50-62.

139. Yao JS, Zhai W, Young WL, Yang GY. Interleukin-6 triggers human cerebral endothelial cells proliferation and migration: the role for KDR and MMP-9. Biochem Biophys Res Commun. 2006;342:1396-404.

140. Yue TL, Wang X, Sung CP et al. Interleukin-8. A mitogen and chemoattractant for vascular smooth muscle cells. Circ Res. 1994;75:1-7.

141. Choi JW, Kim S, Kim TM et al. Basic fibroblast growth factor activates MEK/ERK cell signaling pathway and stimulates the proliferation of chicken primordial germ cells. PLoS One. 2010;5:e12968.

142. Kayakabe K, Kuroiwa T, Sakurai N et al. Interleukin-6 promotes destabilized angiogenesis by modulating angiopoietin expression in rheumatoid arthritis. Rheumatology (Oxford). 2012;51:1571-9.

143. Li A, Dubey S, Varney ML, Dave BJ, Singh RK. IL-8 directly enhanced endothelial cell survival, proliferation, and matrix metalloproteinases production and regulated angiogenesis. J Immunol. 2003;170:3369-76.

144. Sgadari C, Angiolillo AL, Tosato G. Inhibition of angiogenesis by interleukin-12 is mediated by the interferon-inducible protein 10. Blood. 1996;87:3877-82.

145. Takada K, Nakane T, Masuda K, Ishii H. Ursolic acid and oleanolic acid, members of pentacyclic triterpenoid acids, suppress TNF-alpha-induced E-selectin expression by cultured umbilical vein endothelial cells. Phytomedicine. 2010;17:1114-9.

146. Conconi MT, Nico B, Guidolin D et al. Ghrelin inhibits FGF-2-mediated angiogenesis in vitro and in vivo. Peptides. 2004;25:2179-85.

147. Kim MP, Evans DB, Wang H, Abbruzzese JL, Fleming JB, Gallick GE. Generation of orthotopic and heterotopic human pancreatic cancer xenografts in immunodeficient mice. Nat Protoc. 2009;4:1670-80.

148. Seaman ME, Peirce SM, Kelly K. Rapid analysis of vessel elements (RAVE): a tool for studying physiologic, pathologic and tumor angiogenesis. PLoS One. 2011;6:e20807.

149. Carmeliet P, Jain RK. Principles and mechanisms of vessel normalization for cancer and other angiogenic diseases. Nat Rev Drug Discov. 2011;10:417-27.

150. Doukas CN, Maglogiannis I, Chatziioannou AA. Computer-supported angiogenesis quantification using image analysis and statistical averaging. IEEE Trans Inf Technol Biomed. 2008;12:650-7. 
151. Lin KY, Maricevich M, Bardeesy N, Weissleder R, Mahmood U. In vivo quantitative microvasculature phenotype imaging of healthy and malignant tissues using a fiber-optic confocal laser microprobe. Transl Oncol. 2008;1:84-94.

152. Yonenaga $\mathrm{Y}$, Mori A, Onodera $\mathrm{H}$ et al. Absence of smooth muscle actin-positive pericyte coverage of tumor vessels correlates with hematogenous metastasis and prognosis of colorectal cancer patients. Oncology. 2005;69:159-66.

153. Hamzah J, Jugold M, Kiessling F et al. Vascular normalization in Rgs5-deficient tumours promotes immune destruction. Nature. 2008;453:410-4.

154. O'Connor JP, Jackson A, Parker GJ, Jayson GC. DCE-MRI biomarkers in the clinical evaluation of antiangiogenic and vascular disrupting agents. Br J Cancer. 2007;96:189-95.

155. Turkbey B, Thomasson D, Pang Y, Bernardo M, Choyke PL. The role of dynamic contrast-enhanced MRI in cancer diagnosis and treatment. Diagn Interv Radiol. 2010;16:186-92.

156. Nakamura K, Taguchi E, Miura T et al. KRN951, a highly potent inhibitor of vascular endothelial growth factor receptor tyrosine kinases, has antitumor activities and affects functional vascular properties. Cancer Res. 2006;66:9134-42.

157. Nosov DA, Esteves B, Lipatov ON et al. Antitumor activity and safety of tivozanib (AV-951) in a phase II randomized discontinuation trial in patients with renal cell carcinoma. J Clin Oncol. 2012;30:1678-85.

158. Christian TF, Rettmann DW, Aletras AH et al. Absolute myocardial perfusion in canines measured by using dual-bolus first-pass MR imaging. Radiology. 2004;232:677-84.

159. Tofts PS, Brix G, Buckley DL et al. Estimating kinetic parameters from dynamic contrast-enhanced T(1)-weighted MRI of a diffusable tracer: standardized quantities and symbols. J Magn Reson Imaging. 1999;10:223-32.

160. Gaustad JV, Brurberg KG, Simonsen TG, Mollatt CS, Rofstad EK. Tumor vascularity assessed by magnetic resonance imaging and intravital microscopy imaging. Neoplasia. 2008;10:354-62.

161. Wedam SB, Low JA, Yang SX et al. Antiangiogenic and antitumor effects of bevacizumab in patients with inflammatory and locally advanced breast cancer. J Clin Oncol. 2006;24:769-77.

162. de Lussanet QG, van Golde JC, Beets-Tan RG et al. Dynamic contrast-enhanced MRI of muscle perfusion combined with MR angiography of collateral artery growth in a femoral artery ligation model. NMR Biomed. 2007;20:717-25.

163. Shishodia S, Amin HM, Lai R, Aggarwal BB. Curcumin (diferuloylmethane) inhibits constitutive NF-kappaB activation, induces $\mathrm{G} 1 / \mathrm{S}$ arrest, suppresses proliferation, and induces apoptosis in mantle cell lymphoma. Biochem Pharmacol. 2005;70:700-13.

164. Zieba M, Suwalski M, Kwiatkowska S et al. Comparison of hydrogen peroxide generation and the content of lipid peroxidation products in lung cancer tissue and pulmonary parenchyma. Respir Med. 2000;94:800-5. 
165. Lu T, Gabrilovich DI. Molecular pathways: tumor-infiltrating myeloid cells and reactive oxygen species in regulation of tumor microenvironment. Clin Cancer Res. 2012;18:4877-82.

166. Szatrowski TP, Nathan CF. Production of large amounts of hydrogen peroxide by human tumor cells. Cancer Res. 1991;51:794-8.

167. Burdon RH. Superoxide and hydrogen peroxide in relation to mammalian cell proliferation. Free Radic Biol Med. 1995;18:775-94.

168. Gong G, Qin Y, Huang W, Zhou S, Yang X, Li D. Rutin inhibits hydrogen peroxideinduced apoptosis through regulating reactive oxygen species mediated mitochondrial dysfunction pathway in human umbilical vein endothelial cells. Eur J Pharmacol. 2010;628:27-35.

169. Jiang B, Liang P, Zhang B, Song J, Huang X, Xiao X. Role of PPAR-beta in hydrogen peroxide-induced apoptosis in human umbilical vein endothelial cells. Atherosclerosis. 2009;204:353-8.

170. Rayet B, Gelinas C. Aberrant rel/nfkb genes and activity in human cancer. Oncogene. 1999;18:6938-47.

171. Kwon O, Kim KA, Kim SO et al. NF-kappaB inhibition increases chemosensitivity to trichostatin A-induced cell death of Ki-Ras-transformed human prostate epithelial cells. Carcinogenesis. 2006;27:2258-68.

172. Kassim SK, Ali HS, Sallam MM et al. Increased bcl-2 expression is associated with primary resistance to chemotherapy in human epithelial ovarian cancer. Clin Biochem. 1999;32:333-8.

173. Sartorius UA, Krammer PH. Upregulation of Bcl-2 is involved in the mediation of chemotherapy resistance in human small cell lung cancer cell lines. Int J Cancer. 2002;97:584-92.

174. Del Poeta G, Venditti A, Del Principe MI et al. Amount of spontaneous apoptosis detected by Bax/Bcl-2 ratio predicts outcome in acute myeloid leukemia (AML). Blood. 2003;101:2125-31.

175. Shin HM, Kim MH, Kim BH et al. Inhibitory action of novel aromatic diamine compound on lipopolysaccharide-induced nuclear translocation of NF-kappaB without affecting IkappaB degradation. FEBS Lett. 2004;571:50-4.

176. Qiao L, Yu J, Dent P, Farrell G. NF-kappaB protects rat ARL-6 hepatocellular carcinoma cells against hydrogen peroxide-induced apoptosis. Cancer Biol Ther. 2005;4:1195-202.

177. Scott ML, Fujita T, Liou HC, Nolan GP, Baltimore D. The p65 subunit of mechanisms.NF-kappa B regulates I kappa B by two distinct. Genes Dev. 1993;7:1266-76.

178. Chen F, Castranova V, Shi X. New insights into the role of nuclear factor-kappaB in cell growth regulation. Am J Pathol. 2001;159:387-97.

179. Viatour P, Bentires-Alj M, Chariot A et al. NF- kappa B2/p100 induces Bcl-2 expression. Leukemia. 2003;17:1349-56. 
180. Nguyen M, Millar DG, Yong VW, Korsmeyer SJ, Shore GC. Targeting of Bcl-2 to the mitochondrial outer membrane by a $\mathrm{COOH}$-terminal signal anchor sequence. $\mathrm{J}$ Biol Chem. 1993;268:25265-8.

181. Youle RJ, Strasser A. The BCL-2 protein family: opposing activities that mediate cell death. Nat Rev Mol Cell Biol. 2008;9:47-59.

182. Dunn KW, Kamocka MM, McDonald JH. A practical guide to evaluating colocalization in biological microscopy. Am J Physiol Cell Physiol. 2011;300:C723-42.

183. Potts BC, Smith J, Akke M et al. The structure of calcyclin reveals a novel homodimeric fold for S100 Ca(2+)-binding proteins. Nat Struct Biol. 1995;2:790-6.

184. Leukert N, Vogl T, Strupat K, Reichelt R, Sorg C, Roth J. Calcium-dependent tetramer formation of S100A8 and S100A9 is essential for biological activity. J Mol Biol. 2006;359:961-72.

185. Heizmann CW, Cox JA. New perspectives on S100 proteins: a multi-functional $\mathrm{Ca}(2+)-, \mathrm{Zn}(2+)-$ and $\mathrm{Cu}(2+)$-binding protein family. Biometals. 1998;11:383-97.

186. Rustandi RR, Drohat AC, Baldisseri DM, Wilder PT, Weber DJ. The Ca(2+)dependent interaction of $\mathrm{S} 100 \mathrm{~B}$ (beta beta) with a peptide derived from $\mathrm{p} 53$. Biochemistry. 1998;37:1951-60.

187. Deloulme JC, Gentil BJ, Baudier J. Monitoring of S100 homodimerization and heterodimeric interactions by the yeast two-hybrid system. Microsc Res Tech. 2003;60:560-8.

188. Markova NG, Marekov LN, Chipev CC, Gan SQ, Idler WW, Steinert PM. Profilaggrin is a major epidermal calcium-binding protein. Mol Cell Biol. 1993;13:613-25.

189. Presland RB, Bassuk JA, Kimball JR, Dale BA. Characterization of two distinct calcium-binding sites in the amino-terminus of human profilaggrin. J Invest Dermatol. 1995; 104:218-23.

190. Dallwig R, Deitmer JW. Cell-type specific calcium responses in acute rat hippocampal slices. J Neurosci Methods. 2002;116:77-87.

191. Bharti AC, Donato N, Singh S, Aggarwal BB. Curcumin (diferuloylmethane) downregulates the constitutive activation of nuclear factor-kappa B and IkappaBalpha kinase in human multiple myeloma cells, leading to suppression of proliferation and induction of apoptosis. Blood. 2003;101:1053-62.

192. Voboril R, Weberova-Voborilova J. Constitutive NF-kappaB activity in colorectal cancer cells: impact on radiation-induced NF-kappaB activity, radiosensitivity, and apoptosis. Neoplasma. 2006;53:518-23.

193. Li F, Sethi G. Targeting transcription factor NF-kappaB to overcome chemoresistance and radioresistance in cancer therapy. Biochim Biophys Acta. 2010;1805:167-80.

194. Israel A. The IKK complex, a central regulator of NF-kappaB activation. Cold Spring Harb Perspect Biol. 2010;2:a000158.

195. Chen BC, Lin WW. PKC- and ERK-dependent activation of I kappa B kinase by lipopolysaccharide in macrophages: enhancement by $\mathrm{P} 2 \mathrm{Y}$ receptor-mediated CaMK activation. Br J Pharmacol. 2001;134:1055-65. 
196. Tam AB, Mercado EL, Hoffmann A, Niwa M. ER stress activates NF-kappaB by integrating functions of basal IKK activity, IRE1 and PERK. PLoS One.

2012;7:e45078.

197. Zhou M, Gu L, Zhu N, Woods WG, Findley HW. Transfection of a dominantnegative mutant NF- $\kappa \mathrm{B}$ inhibitor $(\mathrm{IkBm})$ represses $\mathrm{p} 53$-dependent apoptosis in acute lymphoblastic leukemia cells: interaction of $\mathrm{IkBm}$ and $\mathrm{p} 53$. Oncogene. 2003;22:8137-44.

198. Piva R, Belardo G, Santoro MG. NF-kappaB: a stress-regulated switch for cell survival. Antioxid Redox Signal. 2006;8:478-86.

199. Donato R. S100: a multigenic family of calcium-modulated proteins of the EF-hand type with intracellular and extracellular functional roles. Int J Biochem Cell Biol. 2001;33:637-68.

200. Bergers G, Hanahan D. Modes of resistance to anti-angiogenic therapy. Nat Rev Cancer. 2008;8:592-603.

201. Takaishi M, Makino T, Morohashi M, Huh NH. Identification of human hornerin and its expression in regenerating and psoriatic skin. J Biol Chem. 2005;280:4696-703.

202. Makino T, Takaishi M, Toyoda M, Morohashi M, Huh NH. Expression of hornerin in stratified squamous epithelium in the mouse: a comparative analysis with profilaggrin. J Histochem Cytochem. 2003;51:485-92.

203. Makino T, Takaishi M, Morohashi M, Huh NH. Hornerin, a novel profilaggrin-like protein and differentiation-specific marker isolated from mouse skin. J Biol Chem. 2001;276:47445-52.

204. Fleming JM, Ginsburg E, Oliver SD, Goldsmith P, Vonderhaar BK. Hornerin, an S100 family protein, is functional in breast cells and aberrantly expressed in breast cancer. BMC Cancer. 2012;12:266. 